\title{
Emission estimation based on traffic models and measurements
}

Nikolaos Tsanakas 


\title{
Emission estimation based on traffic models and measurements
}

\author{
Nikolaos Tsanakas
}

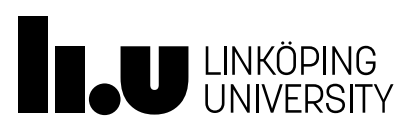

Department of Science and Technology

Linköping University, SE-601 74 Norrköping, Sweden 
Emission estimation based on traffic models and measurements Nikolaos Tsanakas

LIU-TEK-LIC 2019

ISBN 978-91-7685-092-3

ISSN $0280-7971$

Linköping University

Department of Science and Technology

SE-601 74 Norrköping

Printed by LiU Tryck, Linköping, Sweden 2019 


\section{Abstract}

Traffic congestion increases travel times, but also results in higher energy usage and vehicular emissions. To evaluate the impact of traffic emissions on environment and human health, the accurate estimation of their rates and location is required. Traffic emission models can be used for estimating emissions, providing emission factors in grams per vehicle and kilometre. Emission factors are defined for specific traffic situations, and traffic data is necessary in order to determine these traffic situations along a traffic network. The required traffic data, which consists of average speed and flow, can be obtained either from traffic models or sensor measurements.

In large urban areas, the collection of cross-sectional data from stationary sensors is a cost-efficient method of deriving traffic data for emission modelling. However, the traditional approaches of extrapolating this data in time and space may not accurately capture the variations of the traffic variables when congestion is high, affecting the emission estimation. Static transportation planning models, commonly used for the evaluation of policy changes, constitute an alternative efficient method of estimating the traffic data. Nevertheless, their static nature may result in an inaccurate estimation of dynamic traffic variables, such as the location of congestion, having a direct impact on emission estimation. Congestion is strongly correlated with increased emission rates, and since emissions have location specific effects, the location of congestion becomes a crucial aspect.

Therefore, the derivation of traffic data for emission modelling usually relies on the simplified, traditional approaches. The aim of this thesis is to identify, quantify and finally reduce the errors that these traditional approaches introduce in an emission analysis.

According to our main findings, traditional approaches may be sufficient for analysing pollutants with global effects such as $\mathrm{CO}_{2}$, or for large-scale emission modelling applications such as emission inventories. However, for more temporally and spatially sensitive applications, such as dispersion and exposure modelling, a more detailed approach is needed. In case of cross-sectional measurements, we suggest and evaluate the use of a more detailed, but computationally more expensive, data extrapolation approach. Additionally, considering the inabilities of static models, we propose and evaluate the post-processing of their results, by applying quasi-dynamic network loading. 



\section{Acknowledgments}

First and foremost, I would like to thank my supervisor Jan Lundgren and my co-supervisors, Joakim Ekström and Johan Olstam, for their continuous scientific guidance, generous support and enthusiastic encouragement during my studies. Their profound knowledge, experience and useful comments made this thesis possible. Having the opportunity to collaborate with them has been a great honour.

I gratefully acknowledge the funding source of my research work. The research in this thesis is part of the BE3AT project (Better Estimations of Energy use and Emissions when Analysing Traffic) funded by the Swedish Energy Agency (grant number 38921-1). I would like to acknowledge all the members of the BE3AT project.

I would like to offer my special thanks to all my colleagues at the division of Communications and Transportation systems, and especially to the traffic research group for all the rewarding discussions. I would also like to thank Viveka Nilsson and the rest of administrative stuff for their valuable assistance.

I am grateful to all my friends here in Norrköping for supporting me even in the toughest moments. Maria, Elina, Yanni A., Yanni P., Christo, Mano, Niko, Eleni, Giorgo, Cristian, Wenjian and Alan thank you for all the beautiful moments we spent together. Special thanks to my office mates, Angela and Marcus, for making my work time more pleasant. I would like to express my gratitude to my dear friends in Greece and off course to Elena for supporting me spiritually throughout writing this thesis and my life in general.

Above all, I would like to thank my parents, Thanasi and Theodora, my brother Antoni and my sister Elena whose love and support are with me in whatever I pursue.

I would like to dedicate this thesis to my grandfathers, Niko and Antoni, since I didn't have the opportunity to say a proper goodbye to them.

Norrköping, March 2019

Nikolaos Tsanakas 



\section{Abbreviations}

AADS Annual Average Daily Speed

AADT Annual Average Daily Traffic

AHS Average Homogeneous Segment

AOI Area Of Influence

ASM Adaptive Smoothing Method

AVI Automated Vehicle Identification

BPR Bureau of Public Roads function

COPERT COmputer Programme to calculate Emissions from Road Transport

CTM Cell Transmission Model

DSD Disaggregate Simplicial Decomposition

DTA Dynamic Traffic Assignment

DUE Dynamic User Equilibrium

ECEP Excess due to Congestion Emissions Percentage

eLTM event-based Link Transmission Model

ESTA Errors Due to Spatial and Temporal Aggregation

EU European Union

FIFO First In First Out

FCD Floating Car Data 
GPS Global Positioning Systems

HBEFA HandBook on Emissions FActors for road transport

ITS Intelligent Transportation Systems

LTM Link Transmission Model

LWR Lighthill, Whitham and Richards

MW Motorway

O-D Origin-Destination

PC-C Passenger Cars Diesel

PC-P Passenger Cars Petrol

PGE People Grams Exposure

PTC Permanent Traffic Counters

SPTC Short Period Traffic Counts

STA Static Traffic Assignment

STAQ Static Traffic Assignment with Queuing

UE User Equilibrium

UNFCCC United Nations Framework Convention on Climate Change

NEI National Emission Inventories

URB Urban

V/C Volume Capacity Ratio

VDF Volume Delay Function

UNECE United Nations Economic Commission for Europe 


\section{Contents}

Abstract

Acknowledgments $\quad \mathrm{V}$

Abbreviations vii

1 Introduction 1

1.1 Background 1

1.2 Problem definition 2

1.3 Aims and research questions 5

1.4 Method and delimitations 6

$\begin{array}{lll}1.5 & \text { Contributions } & 7\end{array}$

1.6 Outline 9

2 Theoretical background 11

2.1 Emissions from road traffic 11

2.1.1 Categories and classification of effects 13

2.1.2 Emission Modelling 16

2.1.3 Applications of emission data 18

2.2 Traffic Modelling 21

2.2.1 Traffic flow modelling 22

2.2.2 Transportation planning models $\quad 27$

2.3 Traffic measurements 37

2.3.1 Measurements types and devices 37

2.3.2 Traffic state estimation 39

3 Emission estimation based on traffic measurements $\quad 45$

3.1 Review of the methods 46

3.1.1 Approaches based on trajectory data 46

3.1.2 Approaches based on cross-sectional data 47 
3.2 Limitations associated with cross-sectional data 48

3.3 Comparison of the traffic estimation methods 49

3.4 Computational results 55

3.5 Discussion 63

4 Emission estimation based on static traffic models $\quad 65$

4.1 Definition and categorisation of the problems 66

4.2 Quantification of the errors 67

4.2.1 Methodology 68

4.2.2 Errors due to spatio-temporal aggregation $\quad 69$

4.2.3 Errors due to static loading 72

$\begin{array}{lll}4.3 \text { Discussion } & 75\end{array}$

5 Improved emission estimation through post-processing 77

5.1 Post-processing of static modelling results 78

5.2 Quasi-dynamic network loading as a post-processing $\begin{array}{ll}\text { approach } & 79\end{array}$

5.3 Computational results $\quad 83$

$\begin{array}{ll}\text { 5.3.1 Case study } & 83\end{array}$

5.3.2 Network effects $\quad 84$

5.4 Discussion 93

6 Conclusions and Future research 95

6.1 Conclusions 95

6.2 Future research 97

Appendices $\quad 99$

A Vehicle fleet composition in Sweden and HBEFA emission factors 101

B Description of the modified STAQ algorithm 105

B.1 Squeezing phase 106

$\begin{array}{ll}\text { B.2 Queuing phase } & 108\end{array}$

$\begin{array}{ll}\text { Bibliography } & 118\end{array}$ 


\section{Chapter 1}

\section{Introduction}

\subsection{Background}

The growing need of travelling, which is a main characteristic of our modern society, has led to highly congested urban areas. Traffic congestion increases travel times, but also results in higher energy usage and vehicular emissions. Therefore, the continuous traffic growth is associated with negative environmental effects, which are related to both air quality and climate change. According to the European Environment Agency, transportation emissions, and especially exhaust emissions from road traffic, remains a significant contributor to the main air pollutants affecting substantially the urban air quality (EEA, 2016a). Specifically, $\mathrm{NO}_{\mathrm{x}}, \mathrm{CO}$ and $\mathrm{PM}_{2.5}$ make up $32 \%, 23 \%$ and $8 \%$ of the total emissions, respectively. This vigorous effect of vehicular emissions to the urban air quality, raises concerns over the impact of traffic on global climate and human health. Therefore, an effective implementation of emission reducing policies, such as traffic control measures or congestion pricing, becomes crucial for many European cities in order to meet the air quality standards and mitigate the human exposure to pollution. By taking the correct decisions and making the appropriate interventions in traffic, transportation planners and policy makers can alleviate congestion and hence reduce its negative impacts.

To quantify the environmental effects and demonstrate the effectiveness of these interventions, a reliable estimation of pollutant concentrations is needed. The estimation of pollutants concentrations 


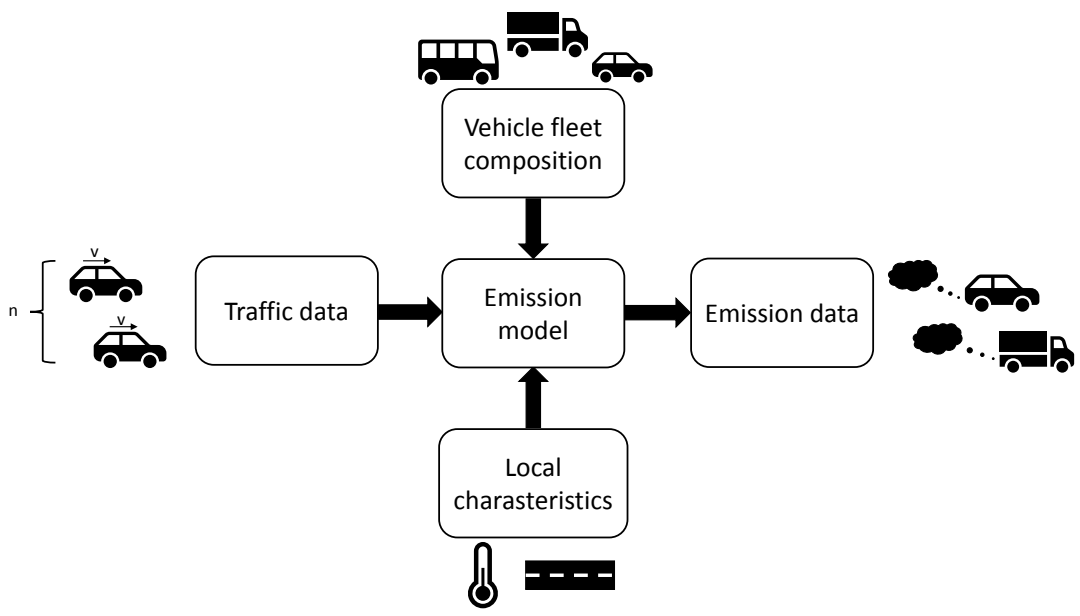

Figure 1.1: The emission data estimation procedure. The input traffic data commonly includes the number of vehicles $n$ and their average speed $\vec{v}$, while the resulting emission data is expressed in grams of pollutant emitted per kilometre.

is performed by air quality modelling, where transportation emissions are combined with emissions coming from other sources and meteorological data. One important air quality modelling step is the estimation of road emissions at a street level. For this step, traffic emission models are used, employing traffic related data (speed and flow), information on vehicle fleet and other local characteristics (e.g. road type and gradient, ambient temperature) (see Figure 1.1). The basic aim of these models is to estimate the appropriate amount of a pollutant emitted in grams per vehicle and kilometre. In large urban areas, macroscopic or aggregate emission models are commonly applied, with COPERT (Ntziachristos et al., 2009) and HBEFA (Keller, 2010) being the two leading emission models in Europe.

\subsection{Problem definition}

One of the most significant inputs to emission models is the traffic data, which consists of the traffic state and traffic activity. Traffic state can be determined by the average speed, flow and density, while traffic activity regards the traffic flow over a specific time period. Traffic data can be obtained from traffic models (Borge et al., 2012; 
Namdeo et al., 2002; Wismans et al., 2013), measurements from traffic sensors (Beevers and Carslaw, 2005; Jing et al., 2016; Johansson et al., 2009), or from combination between models and measurements (Batterman et al., 2014; Gately et al., 2017). However, for large scale applications, emission estimation analyses, relying on either traffic models or measurements, are commonly based on important simplifications regarding the input traffic data.

These simplifications are mainly associated with the spatial and temporal resolution of traffic data considered in both traffic models and measurements. Traffic state and activity are usually derived from aggregation in time and space and expressed by average speeds and traffic flows. In contrast to the travel time-average speed relationship, the relationship between emissions and average speed is non-linear, making emission modelling sensitive speed variations. Hence, long aggregation intervals can lead to inaccurate results both in terms of total emission rates and spatio-temporal distribution of the emitted pollutants. Nevertheless, aggregation may have a minor effect when emission data is used for long-term policies such as annual reporting of total national emissions. In this case, other parameters such as vehicle fleet composition can be more influential. However, aggregate emission data is commonly used in other applications such as air quality modelling (see Figure 1.2), which are more sensitive to spatio-temporal variations of emission rates. Therefore, simplifications regarding the variation in traffic conditions affect emission data estimation, which may lead to unreliable results in many applications of emission data.

Concerning the case where traffic models are used to generate the traffic data, in large urban areas emission models usually rely on traffic data attained from macroscopic transport planning models. Specifically, traffic assignment models, such as Static Traffic Assignment (STA) models, are mainly used to generate inputs for emissions modelling, since they can efficiently be applied to larger areas with relatively low computational cost. STA models are used at a strategic level in order to assess long-term effects of policy measures or changes in the transportation infrastructure. However, their underlying assumptions, related to long aggregation intervals, can lead to inaccurate predictions when variations in traffic conditions are high. Additionally, by using static models, many important dynamic traffic flow phenomena, such as the formation and the propagation of queues and spill-back, are not taken into account. This can result in 


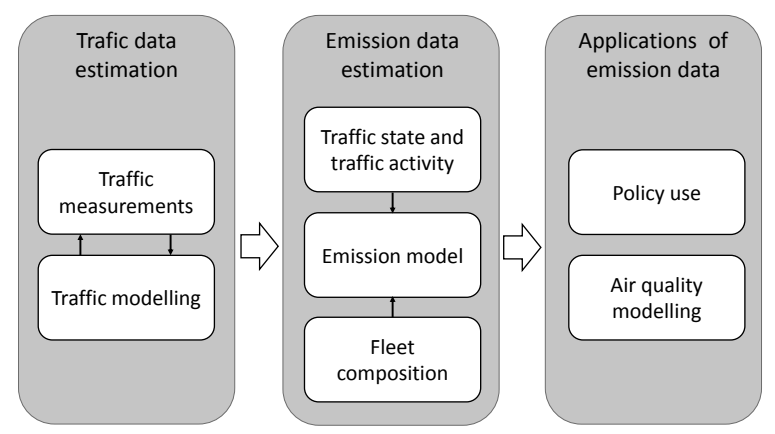

Figure 1.2: The same traffic data input is commonly used for policy use applications of emission data such as the annual report of national emissions, but also for more temporally and spatially-sensitive applications, such as dispersion and exposure modelling.

an inaccurate estimation of dynamic variables, such as the location of congestion.

When the basic output of a traffic analysis is travel time, the accuracy of congestion's location may not be so crucial, since the total origin-destination travel times could be unaffected. However, during an emission estimation analysis, the accurate determination of the level and the location of congestion is essential, since many applications of emission data such as air quality modelling have location specific effects. Therefore, the spatial average travel times and consequently the average link speeds, computed by STA models, may be insufficient to emission modelling, especially in congested networks.

While static models describe a mean traffic state over a long time horizon, dynamic modelling approaches, such as Dynamic Traffic Assignment (DTA) allow the traffic conditions to be described for shorter time periods. In addition, spill-back of the queues as traffic demand exceeds capacity, and dispersion of the queues when demand is below the capacity, can be modelled. However, complexity issues make dynamic modelling computationally expensive and time consuming to calibrate, discouraging their application in larger areas. A middleground solution between static and dynamic modelling could be the post-processing of static models based on quasi-dynamic approaches.

Considering the limitations associated with the use of macroscopic traffic models for emission estimation, many researchers have moved towards the exploitation of the increasingly accessible data from traf- 
fic sensors. This type of data can either be used in combination with traffic models, or on its own as the basis for determining the emission factors. Nevertheless, a comprehensive data set with respect to both traffic state and traffic activity is not always feasible. The available data has to be extrapolated in time and space using methods for missing data imputation, such as traffic state estimation. However, the traditional traffic state estimation methods used in large areas, being originally developed for other applications rather than emission modelling, could lead to considerable emission estimation errors. Such approaches consider assumptions, concerning the variations of traffic conditions in time and space, which potentially can affect the final emission estimates. In contrast to the traditional approaches, more complex traffic state estimators, by accurately capturing the traffic state variations can lead to more reliable emission estimates. However, their use is discouraged for large areas, since they are computationally more expensive than the traditional approaches.

\subsection{Aims and research questions}

The overall aim of the thesis is to identify, quantify and finally reduce the potential errors that unrealistic assumptions regarding the variations in traffic conditions introduce in emission estimation analyses.

More specifically, the thesis aims to address the following research questions:

Q1. Do traditional approaches of extrapolating traffic measurements in time and space lead to less reliable emissions estimates compared to more sophisticated traffic state estimators? How are these differences distributed in time and space and what can be the effects on the applications of emission modelling?

Q2. What are the emission estimation errors resulting due to the simplified assumptions considered in static modelling?

Q3. Can post-processing of static modelling lead to more accurate emission estimation? What would be the effects of post-processing with respect to the various emission modelling applications? 


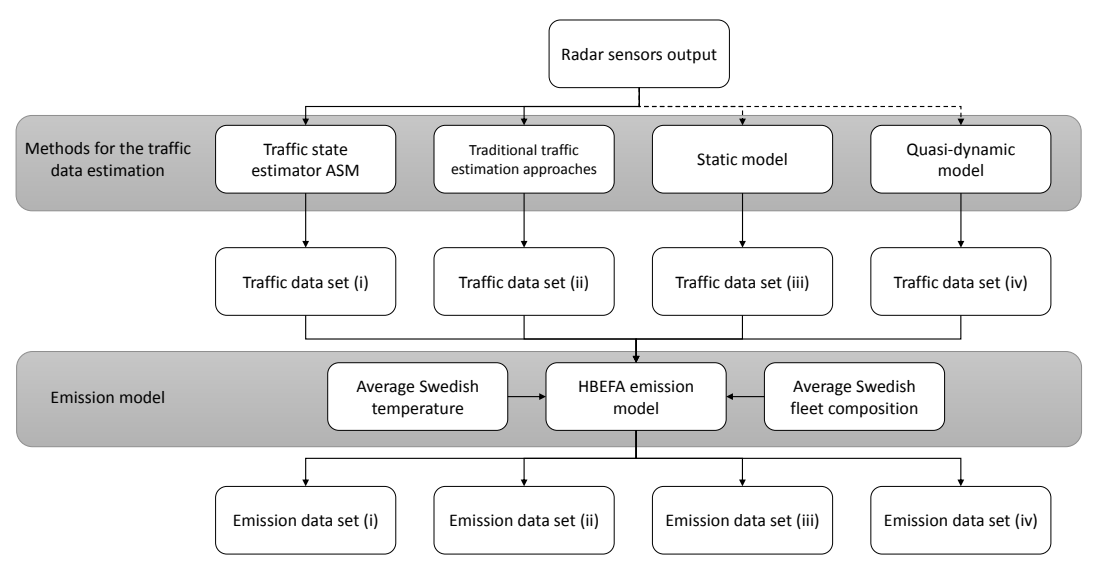

Figure 1.3: The methodology framework used in this thesis.

\subsection{Method and delimitations}

The basic aim of the thesis is to investigate how the different methods of deriving traffic data influence the emission estimation. For that reason, we perform a comparison between different emission data sets attained from various approaches of traffic data estimation. We calculate the input traffic data based on four different approaches (see Figure 1.3), for the same case study motorway segment. Then we evaluate the effects of each approach with respect to emission modelling. For the emission estimation, the HBEFA emission model is used while the case study motorway segment is a part of the E4 highway passing by central Stockholm.

Radar sensors are installed at approximately every 500 meters along the segment, measuring one-minute average speed and flow. Based on these temporally dense measurements we initially fill the spatio-temporal gaps using a traffic state estimator, the Adaptive Smoothing Method (ASM). The resulting traffic data is the traffic data set (i). The resulting emission estimates from traffic data set (i) is the emission data set (i) (see Figure 1.3). The traffic state estimator provides a quite detailed and accurate representation of the real traffic conditions and the resulting emission estimates can be assumed as the ground-truth. Next, considering the same radar sensors measurements, the traffic data is estimated through traditional traffic estimation approaches, such as Annual Average Daily Traffic (AADT), 
resulting in the traffic data set (ii) and the emission data set (ii). By comparing the emission data set (ii) with the emission data set (i) and by evaluating the spatio-temporal distribution of the differences we give an answer to Q1.

The radar sensors measurements are also used to calibrate a static traffic model, which is used to derive the traffic data set (iii) and the corresponding emission data set (iii). Comparing the emission data set(iii) with the emission data set (i) we answer Q2. Next, we apply a quasi-dynamic model, as post-processor to the static model, leading to a new traffic data set (iv) and emission data set (iv). By comparing again the emission data set (iv) with the emission data set (i) and investigating the spatio-temporal allocation of the differences, we answer Q3.

At this point we should highlight the basic delimitations associated with this study. We mainly focus on how the spatio-temporal variations of traffic flow and speed affect an emission estimation, making, though, some important assumptions regarding the other inputs of an emission model such as the vehicle fleet composition and the local characteristics (see Figure 1.1). Although we estimate emissions for a relatively long period, we assume that the vehicle fleet composition and the ambient temperature are constant. In addition, we evaluate the various approaches depicted in Figure 1.3 with respect to air quality, by investigating the spatio-temporal distribution of the road emissions rates, without performing any air quality modelling. We draw some conclusions by assuming that road traffic emissions have a direct effect on air quality.

\subsection{Contributions}

The thesis contributes to the field of road traffic emission estimation, towards many directions. The thesis:

- gives an overview of methodologies for emission estimation from sensors measurements and traffic models outputs,

- identifies problems associated with the assumptions and simplifications considered during an emission estimation analysis,

- quantifies the emission estimation errors resulting from the traditional traffic state estimators, 
- suggests and evaluates the use of a more advanced traffic estimator for the spatio-temporal interpolation of sensor data, which more effectively captures the traffic variations,

- quantifies the errors that static assignment models introduce in the emission estimation,

- suggests and evaluates a method for post-processing static assignment outputs which is developed for emission estimation and relies on a quasi-dynamic model.

Part of the work presented in this thesis have been published in:

- Tsanakas, N., Ekström, J., Olstam, J. (2017). Reduction of errors when estimating emissions based on static traffic model outputs, Transportation Research Procedia 22, 440-449.

- Tsanakas, N., Ekström, J., Olstam, J.(2017). Emission estimation based on cross-sectional traffic data, in: 22nd International Transport and Air Pollution Conference.

- Tsanakas, N., Ekström, J., Olstam, J.(2017). Estimating emissions from static traffic models - problems and solutions. To be submitted to European Transport Research Review.

- Tsanakas, N., Ekström, J., Olstam, J.(2019). Traffic emission estimation based on quasi-dynamic network loading. Submitted for the 8th Symposium of the European Association for Research in Transportation (hEART 2019).

Parts of the thesis has also been presented by the author or the co-authors of the above publications at the following conferences:

- The 19th EURO Working Group on Transportation Meeting (EWGT2016), Istanbul, Turkey, September 2016.

- Nationella Konferensen i transportforskning, Stockholm, October 2017.

- The 22nd International Transport and Air Pollution conference (TAP2017), Zurich, Switzerland, November 2017. 


\subsection{Outline}

In Chapter 2, the theoretical background of the thesis is presented, including an introduction to emission modelling, traffic modelling and traffic data collecting techniques. Chapter 3 initially gives an overview of the methods for estimating emissions from traffic measurements and continues with the description and investigation of the problems that are associated with the emission estimation when cross-sectional traffic data is used. A methodology of estimating emissions from static models outputs is presented in the first part of Chapter 4 . Chapter 4 continues with the description and the quantification of the errors that static model introduce in the emission estimation process. In Chapter 5 an emission estimation based method for post-processing the static models outputs is presented and evaluated. Chapter 6 concludes the thesis, discussing the most important results of the thesis and suggesting directions for further research. 



\section{Chapter 2}

\section{Theoretical background}

This chapter provides a comprehensive description of the theoretical framework behind the methods and the models used in the thesis. It gives a theoretical insight into each step of the methodology framework illustrated in Figure 1.2.

Section 2.1 is related to the last two steps of the methodology framework. In this section a categorisation of the road emissions is performed, and a brief overview of the basic exhaust pollutants is given. Furthermore, Section 2.1 describes the emission models used in practice and presents the main applications of emission modelling.

Section 2.2 regards the first step of the methodology framework and specifically the traffic modelling. Traffic models are categorised in traffic flow models and transportation planning models. Each one of those two types of models is further analysed with the main focus being on how traffic models can generate inputs for emission modelling. Finally, Section 2.3 is also associated with the first step, describing methods for deriving traffic data from measurements. Initially, an overview of measurements types and devices is given. Next, several traffic state estimation approaches from measurements are described. The main focus is on how measurements from sensors can be processed in order to be suitable for emission modelling.

\subsection{Emissions from road traffic}

With air pollution being the top environmental cause of premature death in Europe (EEA, 2014), there is an increased concern about ur- 


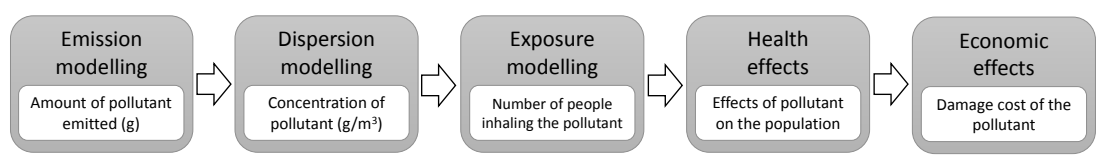

Figure 2.1: Steps required to link source emissions to health effects.

ban air quality. Air pollution is responsible for diseases such as heart disease and stroke leading to premature death, while it increases the incidence of cancer. Besides the negative effects on human health, air pollution can have more global effects, influencing the ecosystem and contributing to the climate change. Although transport sector has considerably reduced its emissions of air pollutants in Europe since 2000 (EEA, 2016a), this sector still remains the largest contributor to emissions such as $\mathrm{NO}_{\mathrm{x}}$. Specifically, only the road transport emissions, which are the main focus of the thesis, account for almost $30 \%$ and $22 \%$ of the total $\mathrm{NO}_{\mathrm{x}}$ and $\mathrm{CO}$ emissions respectively.

Considering these high rates of road emissions and the negative effects of air pollution, someone can clearly conclude that road transport emissions significantly affect human health. To evaluate this effect, many medical research studies are performed, trying to correlate several diseases with the health status of people exposed to road emissions. However, the estimation of the magnitude, the place and the duration of this exposure, requires a pre-process including a sequence of modelling steps (Figure 2.1).

Initially, the amount of a pollutant emitted at a street level for a specific time period in grams is estimated by emission modelling. Next, a dispersion model can be used to provide the concentration of a pollutant in grams per $m^{3}$ at a specific location and time period. Dispersion models describe the chemical and physical processes within a plume combining the resulting from the emission model emission rates, with some other geographical, meteorological and background pollution information. Then, exposure modelling, considering the spatio-temporal information on the pollutant's concentrations as well as demographics and land use data, results in the final number of people inhaling the pollutant. The magnitude of the combined effects on health and environment, determine then the economic effects of air pollution (Smit et al., 2010). Each modelling step depicted in Figure 2.1, will be further described and discussed in Subsections 2.1.2 and 


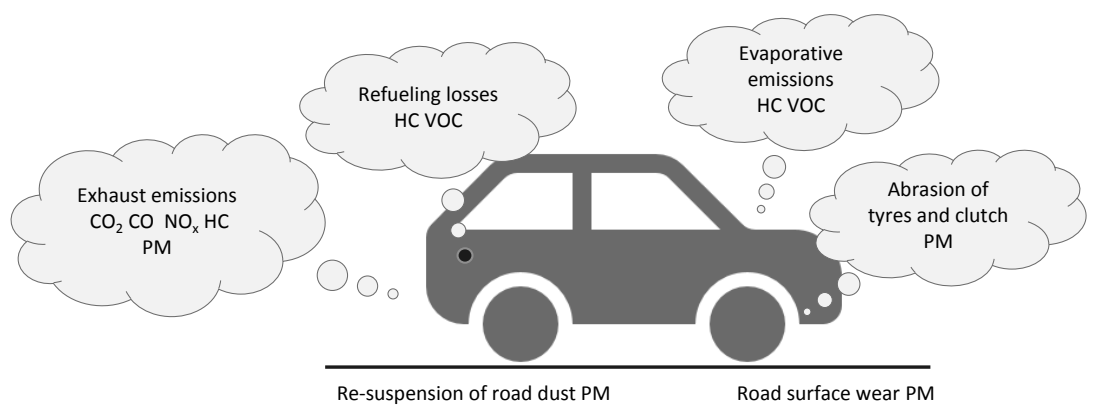

Figure 2.2: The pollutants and greenhouse gases for each emissions type emitted by a vehicle.

\subsection{3.}

\subsubsection{Categories and classification of effects}

The kinetic energy that moves a vehicle comes, in the case of internal combustion vehicles, from the burning fuel in the engine. The emitted pollutants are either products of this combustion process or they come from the evaporation of the fuel itself. Thus, road vehicle emissions can be categorised into 3 different groups (Losses, 1994; Pastorello and Melios, 2016; Keller and Kljun, 2007):

Exhaust emissions. The emissions produced primarily from the combustion of different petroleum products such as petrol, diesel, natural gas and liquefied petroleum gas. These fuels are mixtures of different hydrocarbons, namely compounds that contain hydrogen and carbon atoms. A combustion is said to be "perfect" when oxygen in the air converts all the hydrogen in the fuel to water and all the carbon in the fuel to carbon dioxide, $\mathrm{CO}_{2}$, while Nitrogen in the air remains unaffected. However, in reality, no combustion process is "perfect" and a vehicle can emit some additional pollutants such us $\mathrm{NO}_{\mathrm{x}}$ and $\mathrm{CO}$.

Exhaust emissions can be further divided into two subgroups:

- Hot emissions. These are the emissions produced by a vehicle when the engine is hot. Hot emissions mainly depend on the 
way a vehicle is driven, but as well as on some other parameters, e.g. the gradient of a road section, the fuel and the vehicle type.

- Cold start emissions. Generally, the level of emissions produced with a cold engine differ from hot engine conditions and mostly is higher. Cold start emissions are produced when the engine is still cold and tend towards zero after certain distance driven. They, therefore, depend on the ambient temperature, the parking time before the departure and the distance driven. Similarly to the hot emissions, the level of cold start emissions is influenced by the driving behaviour, the fuel and the vehicle type.

The most important exhaust pollutants and greenhouse gases are presently regulated under the European Union road transport legislation, including:

- Carbon Dioxide $\left(\mathrm{CO}_{2}\right) \cdot \mathrm{CO}_{2}$ is the main product of "perfect" combustion. With respect to air pollution, $\mathrm{CO}_{2}$ is the most important greenhouse gas. Even though, $\mathrm{CO}_{2}$ does not directly harm human health, it is has more global and long-term effects, contributing to the climate change and the global warming.

- Hydrocarbons (HC). HC emissions can be a product of an incomplete or partial combustion, namely when fuel molecules in the engine do not burn or burn only partially. Hydrocarbons in the atmosphere, react with the nitrogen oxides and the sunlight, resulting in the formation of ground-level ozone, a significant component of the photochemical smog. Ozone can cause eyes' irritation and lungs' damages leading to respiratory problems. Some of exhaust hydrocarbons are also toxic, with the potential to cause cancer.

- Nitrogen oxides $\left(\mathrm{NO}_{\mathrm{x}}\right) \cdot \mathrm{NO}_{\mathrm{x}}$ constitutes a group of chemicals formed by the reaction of nitrogen with oxygen under the high pressure and temperature conditions in an engine. Nitrogen oxides also contribute to the formation of ground-level ozone, while they cause the acidification of water resulting in the acid rain.

- Carbon monoxide (CO). When the combustion is incomplete, carbon is only partially oxidised, producing $\mathrm{CO}$ instead of $\mathrm{CO}_{2}$. Although CO is colourless, is extremely toxic, dangerous to people with heart disease, since it reduces the flow of oxygen in the 
bloodstream. In addition, similarly to $\mathrm{HC}$ and $\mathrm{NO}_{\mathrm{x}}, \mathrm{CO}$ is a precursor to the formation of ground-level ozone.

- Particulate matter (PM). PM is also a product of incomplete combustion and constitutes a mixture of both primary and secondary PM. Primary PM is the fraction of PM that is emitted directly into the atmosphere, while secondary PM is formed after the release in the atmosphere of precursor gases. Regarding the human health, PM is one of the most significant pollutants. It can penetrate into sensitive regions of the respiratory system and cause cardiovascular and lung diseases or even cancer.

Vehicles can also emit pollutants which are not currently regulated by the European Union road transport legislation, such as ammonia $\left(\mathrm{NH}_{3}\right)$ and sulphur dioxide $\left(\mathrm{SO}_{2}\right)$, the carcinogenic and toxic polycyclic aromatic hydrocarbons (PAH) and persistent organic pollutants (POP), dioxins, furans and several heavy metals.

Evaporative emissions. This type of emissions is associated with the vapours that escape from the vehicle's fuel system. Evaporative emissions can be distinguished at the following types:

- Diurnal losses. They occur when a vehicle is stationary and the engine is turned off. As the ambient temperature rises and heats the fuel tank, gasoline evaporation becomes higher venting vapours.

- Hot-soak losses. While the engine remains hot for some period after the vehicle has stopped and is parked, gasoline evaporation continues forming the hot-soak losses.

- Running losses. The fuel that is vaporised when the vehicle is running due to the high temperature on the engine and the exhaust system.

- Refueling losses. When the fuel tank is filled with liquid fuel, the saturated vapours are displaced and vented into the atmosphere.

The most significant evaporative emission is the Volatile Organic Compounds (VOC) that can have direct toxic effects to human health, while their indirect effects are associated with the photochemical ozone formation. Petrol fuel vapour also contains a variety of different Hydrocarbons, leading to the negative human health effects discussed above. 
Abrasion emissions. The last group of emissions regards the emissions produced by the mechanical abrasion and corrosion of vehicle parts including the abrasion of the vehicle's tyres, brakes and clutch, the road surface wear and the corrosion of the chassis. The most important abrasion emissions are related to PM, while some heavy metals can also be emitted.

A further detailed description of the on road emission pollutants and their effects to human health can be found in Losses, (1994), Pastorello and Melios, (2016), and Keller and Kljun, (2007).

\subsubsection{Emission Modelling}

The first modelling step depicted in Figure 2.1 regards the emission modelling. One basic component of the emission models is the socalled emission factors. Emission factors are functional relationships of the traffic state, expressing the quantity of a pollutant that a vehicle emits per a specified, depending on the type of the model, distance driven $\left(\mathrm{g} \mathrm{km}^{-1}\right)$, time $\left(\mathrm{g} \mathrm{s}^{-1}\right)$ or mass of fuel burned $\left(\mathrm{g} \mathrm{kg}^{-1}\right)$. The emission factors can be obtained from dynamometer test or other experimental data corresponding to a specific driving cycle. A detailed review of the experimental approaches that have been used in practice for the development of emission factors can be found in Franco et al., (2013).

Depending on the required level of detail of an analysis, emission models can be categorised in microscopic, where emissions are estimated at the level of an individual vehicle, and macroscopic, where emissions are estimated at a road link or a network level. In addition, according to Smit et al., (2009) emission models can be categorised in three types, based on the way that driving behaviour or traffic state is incorporated on the model. The first type regards models that have incorporated speed-time profiles in their development phase, the second is related to the models that generate speed-time profiles as part of the emission modelling process, and the third regards models that require speed-time profiles data as input. The first two types are associated with macroscopic emission models while the third type with microscopic models. 


\section{Microscopic emission models}

Microscopic or instantaneous emission models are used to estimate second by second emissions for individual vehicles, while the emission factors are usually expressed in $g \mathrm{~s}^{-1}$. The two main categories of microscopic emission models are the cycle-variable and the modal models. In cycle-variable models, emission factors are function of various driving cycle variables, such as idle time or average (from minutes to seconds) speed and acceleration. In the case of modal models, emission factors depend on specific engine or vehicle operating modes at a higher resolution, such as second to second speed and acceleration. Both types of models require quite detailed information about the vehicle state. Due to the high level of detail and complexity their application is restricted to small size areas, i.e. specific road sections.

\section{Macroscopic emission models}

Alternatively, macroscopic emission models can be applied on a larger scale, e.g. at a city or a national level. They are also called aggregate emission models, due to the aggregate nature of the input traffic data. The total emissions are calculated by multiplying the traffic activity, usually expressed in vehicle kilometre travelled with the corresponding emission factor expressed in $\mathrm{g} \mathrm{km}^{-1}$. Macroscopic emission models can be categorised, sorted by increasing complexity in average speed models, traffic situation models and traffic variable models (Smit et al., 2010).

Today, COmputer Programme to calculate Emissions from Road Transport (COPERT) and HandBook on Emissions FActors for road transport (HBEFA) are the two leading macroscopic emission models in Europe. COPERT (Ntziachristos et al., 2009) is used by several European countries to officially report their national inventories of emissions from road transport. The emission factors depend on the vehicle class and the road type, and are defined as continuous functions of average speed. HBEFA (Keller, 2010) is an emission factor database which is developed on behalf of several European countries (Germany, Austria, Switzerland, Norway and Sweden). The HBEFA database includes emission factors as a discrete function of vehicle legislative class, road category and traffic situation. The vehicle classes are defined by the exhaust emission limits according to the European emissions standards, while the traffic situations are defined by in- 

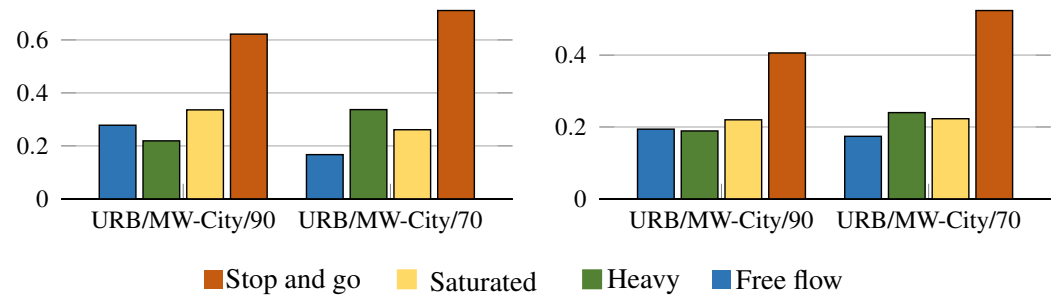

Figure 2.3: HBEFA emission factors for the Swedish road conditions and fleet composition; (a) $\mathrm{CO}_{2}$ (b) $\mathrm{NO}_{\mathrm{x}}$.

dicative flow and speed levels. HBEFA emission factors rely on four traffic situations: Free flow, Heavy, Saturated, and Stop and go. The road categories are divided by road environment (rural and urban), speed limit and road type (Motorway, Trunk road/ Primary, Distributor/ Secondary, Local/ Collector, Access/ Residential). Figure 2.3 illustrates an example of the HBEFA emission factors for two typical pollutants, $\mathrm{CO}$ and $\mathrm{NO}_{\mathrm{x}}$. A detailed description of the two models can be found in Franco et al., (2012).

\subsubsection{Applications of emission data}

By combining traffic data with some other information on road conditions and fleet composition, emission models provide emission data in mass per time period. The resulting emission data, then can be used for several applications and studies such as policy use, air-quality modelling, pre-after studies, evaluation of congestion pricing, ramp metering, traffic light optimisation and other traffic control measures. For a better comprehension of the next chapters, this section discusses some of the basic applications of emission data.

\section{Policy use}

In 1992, considering the threat of global warming and climate change, many countries joined an international treaty, the United Nations Framework Convention on Climate Change (UNFCCC). UNFCCC was a framework for international cooperation to combat the undesired effects of air pollution. The parties of UNFCCC, namely the countries that have ratified the convention, in 1997 adopted the Ky- 
oto Protocol (Protocol, 1997). The Kyoto Protocol legally binds the parties of UNFCCC, which today has near-universal membership, to emission reduction targets. Each country party is obligated to submit annual National Emission Inventories (NEI) to benchmark progress towards the goals of the UNFCCC (Peters, 2008). An emission inventory can be defined as the accounting of the amount of pollutants discharged into the atmosphere in a certain geographical area and within a specified time span, usually a specific year.

Specifically, the member states of European Union (EU) are obligated to also report their NEI, for the United Nations Economic Commission for Europe (UNECE), since EU ratified the UNECE's convention on long-range transboundary air pollution in 1982 (EEA, 2017). The main idea of the convention is associated with the principal that countries cannot protect human health and the environment within their territory by national action alone. Since 1984, eight protocols have come into force, setting the upper limits for each country and pollutant. Member states are then responsible for selecting the activity data, emission factors and other parameters used for their NEI. However, the reported NEI should meet the quality criteria for transparency, consistency, completeness, comparability and accuracy. For this reason, member states should follow the reporting guidelines (UNECE, 2014) and apply the methodologies contained in the EMEP/EEA Guidebook (EEA, 2016b).

Road transport emissions is a significant part of the NEI, with the road transport sector being the major source of the ground-level ozone. According to EEA, (2017), in 2015 road transport sector contributed $38 \%, 21 \%$ and $10 \%$ to total emissions of $\mathrm{NO}_{\mathrm{x}}, \mathrm{CO}$ and nonmethane VOC respectively, in the EU. Since NEI are estimated at a yearly and a national level, emission models usually rely on a quite aggregate in time and space traffic data. The simplifications regarding the spatio-temporal variations of the input traffic data may not significantly influence the resulting NEI. However, this is not true for other applications of the emission data; as it is described in the next sections, they are more sensitive to the spatio-temporal resolution of the traffic data.

\section{Dispersion modelling and air quality}

The estimated emissions derived from the traffic analysis together with the so-called dispersion models determine the air quality at a spe- 
cific location. Dispersion models are based on mathematical models describing the chemical and the physical processes within the plume, calculating pollutant concentrations at specific locations. The traditional dispersion models can be distinguished into four basic families: Box models, Gaussian models, Lagrangian models and Computational fluid dynamic models. A detailed review of the dispersion models that have been used in practice can be found in Holmes and Morawska, (2006).

One significant input considered by the road emissions dispersion models is street geometry or in terms of dispersion modelling the canyon geometry. As street canyon can be defined as a street with buildings lined up continuously along both sides (Nicholson, 1975). The canyon geometry is characterized by the so-called aspect ratio, which is the height of the canyon divided by the width. Generally, high aspect ratios are associated with increased level of pollution because the natural ventilation is reduced (Vardoulakis et al., 2003).

Meteorological conditions are also an important factor affecting the dispersion of the vehicular emissions. Depending on the wind speed (magnitude and direction) and the corresponding aspect ratio, the flow inside a street canyon can be isolated roughness, wake interference or skimming (Oke, 1988). Next, by taking into account the background pollution, dispersion models result in the final concentration of each pollutant in $\mathrm{g} / \mathrm{m}^{3}$ at each location. Since dispersion modelling is sensitive to the local street geometry as well as to local and temporal meteorological conditions and background pollution, the input to the emission model, traffic data, should also be detailed and spatio-temporally disaggregated.

\section{Exposure modelling}

With the distribution of a pollutant's concentration in time and space known, exposure modelling estimates the human exposure to the pollutant. The term exposure was first defined in Ott, (1982) as: "an event that occurs when a person comes in contact with the pollutant". The basic aim of the exposure models is to estimate the number of people being exposed to a pollutant and potentially will inhale the pollutant, as well as the duration and the place that this exposure occurred. The output of exposure studies can be subject of epidemiologic studies, where the emission effects are assessed by correlating the symptoms of a group of people with their exposure to air pollution. 
An important concept usually used in exposure studies is the $m i$ croenvironment defined as a "chunk of air space with homogeneous pollutant concentration" (Duan, 1982). The personal exposure can then be derived by the time fraction each individual spent in each microenvironment (Monn, 2001). While concentrations of air pollutants are subject to high temporal variability, every individual also has unique activity patterns. Thus, exposures take place over time and in multiple location, and for this reason many studies have highlighted the difficulty to accurately estimate the relevant exposures (Zeger et al., 2000; Steinle et al., 2013).

Depending on the required level of detail, exposure studies can either rely on the simplified traditional location-based, proximity methods or on more sophisticated dynamic modelling techniques, including modelling of time-activity patterns. According to the traditional methods, the distribution of the population is static (Steinle et al., 2013), while the exposure only depends on the distance from the source (Jerrett et al., 2005). More complicated methods combine the place of residence with time-activity models (Briggs, 2005; Gulliver and Briggs, 2011). Independently on the applied method, Geographical Information Systems (GIS) offer powerful tools commonly used in practice for exposure assessment.

Considering the nature of the exposure modelling techniques, we can conclude that exposure heavily rely on the spatio-temporal distribution of both population and pollutant concentrations. By not taking into account the variations of traffic conditions and using aggregate traffic data as input to the emission modelling can potentially lead to inaccurate exposure. This can have a direct effect to economic analyses, such cost-benefit analyses, where the damage cost of the emitted pollutants strongly depends on the number of people exposed and affected (Eliasson, 2009). Therefore, from an exposure modelling point of view, traffic data should incorporate the corresponding spatial and temporal traffic variations.

\subsection{Traffic Modelling}

The required input to an emission model, traffic data, can be derived from traffic models. A vehicular traffic network as a system can be placed between a social and a physical system. It can be described by mathematical equations and physics laws, however it is 
always affected by the human factor, such as the driving behaviour or the households' decisions. Traffic models are mathematical models designed to describe phenomena that can affect the operation of a vehicular traffic network. A categorisation among the different traffic models may be developed using various dimensions such as the level of detail in the analysis, the purpose that are used for, the planning horizon or the capability of describing dynamics phenomena.

Traffic models can be used either for the strategic assessment of road network improvements or for the management of the current infrastructure, optimising the flow. Therefore, based on the scope of modelling a distinction can be done between traffic strategic planning models and traffic operational management models. The former have a long planning horizon and the traffic variables are expressed as averages over relatively long time and space intervals. The later usually give short-term prediction and they can be based on microscopic simulation. In the thesis we present the traffic models following the categorisation proposed in Maerivoet and De Moor, (2005), where traffic models are classified into traffic flow models and transportation planning models. On one hand, traffic flow models, described in Subsection 2.2.1, attempt to describe the physical propagation of traffic flows in a road network. On the other hand, transportation planning or network models, presented in Subsection 2.2.2, are associated with households that make certain decisions, such as transport mode or route choice, which lead to transportation and use of infrastructure.

\subsubsection{Traffic flow modelling}

Traffic flow models have been developed and used to understand, describe and predict how traffic flow propagates through a road network since Greenshields' study of traffic capacity in 1934 (Greenshields, 1934). Greenshields proposed the fundamental diagram of traffic, where traffic flow is a function of traffic density. In 1955, Lighthill and Whitham presented a model where the behaviour of vehicles in traffic flow is similar to the one of particles in a fluid (Lighthill and Whitham, 1955). Since then, traffic flow has been an active subject of research, resulting in a broad scope of models. A comprehensive review and categorisation of traffic flow models can be found in Hoogendoorn, (2001) and in Wageningen-Kessels et al., (2014).

One of the most common classification among the traffic flow models is the distinction between microscopic and macroscopic traffic 
modelling approaches. This categorisation is based on the following aspects: traffic flow in microscopic models is represented by individual driver-vehicle units, while the representation of the traffic flow in macroscopic models is in terms of traffic stream or groups of drivers. The variables most commonly used by macroscopic models are the flow $Q$, the density $\rho$ and the average speed $V$. The most commonly used microscopic variables are the position $x$, the individual speed $\dot{x}$ and acceleration $\ddot{x}$ of each vehicle. Finally, a third approach, the mesoscopic, can be placed in between microscopic and macroscopic, where individual vehicles are not distinguished, but the individual behaviour can be specified, while traffic is represented by groups of traffic entities.

\section{Microscopic models}

Microscopic traffic simulation models attempt to describe the behaviour of individual driver-vehicle units and they are usually used for traffic operational management. The core model of microscopic longitudinal simulation is the car-following model. Car-following models describe the manner in which one vehicle following another one, reacts to its surrounding traffic environment and more specifically to the fluctuations of the leading vehicle's traffic conditions. Equation (2.1) and (2.2) describe the general form of the model, that is a set of coupled ordinary differential equations, with the acceleration $\ddot{x}$ of the following vehicle $\nu-1$ at time $t, \ddot{x}_{\nu-1}(t)$, being a function $a_{c f m}$ of its speed, $\dot{x}_{\nu-1}(t)$, of the speed of the leading vehicle, $\dot{x}_{\nu}(t)$, and of the distance between the two vehicles, $s(t)=x_{\nu-1}(t)-x_{\nu}(t)$,

$$
\begin{aligned}
\dot{x}_{\nu-1}(t) & =\frac{d x_{\nu-1}(t)}{d t} \\
\ddot{x}_{\nu-1}(t) & =a_{c f m}\left(\dot{x}_{\nu-1}, \dot{x}_{\nu}(t), s(t)\right) .
\end{aligned}
$$

The early car following models, such the one proposed in Pipes, (1953), were based on empirical driving rules, with distance $s(t)$, being a linear function of the speed $\dot{x}_{\nu-1}$. Since then, numerous models have been suggested in the literature, including the so-called stimulusresponse (Chandler et al., 1958; Gazis et al., 1961), safe-distance (Gipps, 1981) and psycho-physical models (Wiedemann, 1973; Fritzsche, 1994). Besides the description of the longitudinal movement, microscopic models have been developed to describe the lateral movement, such the ones described in Gipps, (1986) and Ahmed, (1999). 
The basic output of microscopic traffic flow models is the trajectory $x_{\nu}(t)$, namely the position of each vehicle $\nu$ in time. The trajectory data is the most comprehensive traffic data available, since it contains direct or indirect information about the position, speed and acceleration of each individual vehicle. By considering each vehicle individually, the level of detail in microscopic traffic flow models is high, and thus, computing power, memory and calibration requirements for a simulation are extensive. For this reason, the application of microscopic traffic flow simulation is limited to small areas, for instance to an intersection or a motorway segment. Microscopic traffic flow models are usually coupled with microscopic emission models, since they provide the appropriate input trajectory traffic data.

\section{Macroscopic models}

In contrast to microscopic models, macroscopic traffic flow models are commonly used for larger areas and longer analysis time periods. Traffic flow is described at an aggregate level and the drivers' behaviour is assumed to be homogeneous. Therefore, macroscopic traffic models describe collective phenomena such as the evolution of congestion and the propagation of traffic waves (Treiber and Kesting, 2013). They are also called hydrodynamic models, since, are commonly derived from the analogy between the traffic flow and the flow of continuous media such as fluid or gases. The basic dynamic macroscopic variables, in correspondence to fluid-dynamics, are the density $\rho(x, t)$, the flow $Q(x, t)$ and the mean speed $V(x, t)$, with the independent variables being the location $x$ and the time instant $t$. Density refers to the expected number of vehicles per length unit, $x+d x$, at time instant $t$, while flow concerns the expected number of vehicles per time unit, $t+d t$, at the location $x$ (Hoogendoorn, 2001).

Since traffic flow in macroscopic models is treated as a one dimensional compressible fluid, two basic assumptions are considered: traffic flow is conserved and there is a one-to-one relationship between flow and density (Nathan Gartner, 2001). Vehicles conservation is a fundamental aspect considered in macroscopic traffic models relationship, analogous to the fluid conservation law of mass, meaning that at a road segment with finite length, no vehicles appear or disappear other than the ones that enter and exit the road segment. The continuity Equation 2.3, 


$$
\frac{\partial \rho}{\partial t}+\frac{\partial Q}{\partial x}=0
$$

describes how a change in density over time relates to a change in flow over space, in a way that vehicle conservation is ensured. One more fundamental relation, is the Hydrodynamic Flow Relation

$$
Q=\rho V,
$$

where $V$ is the space-mean speed. An assumption considered in Hydrodynamic Flow Relation is that traffic conditions at location $x$ and time $t$ are in a steady-state, $[Q(x, t), \rho(x, t), V(x, t)]$. Considering Equation (2.4), the continuity Equation (2.3) becomes

$$
\frac{\partial \rho}{\partial t}+\frac{\partial \rho V}{\partial x}=0
$$

Lighthill and Whitham, (1955) and Richards, (1956) considered a supplementary to Equation (2.3), static relation, where flow is expressed as a function $Q_{f}$ of density or speed as a function $V_{f}$ of density

$$
\begin{aligned}
& Q(x, t)=Q_{f}(\rho(x, t)) \quad \text { or } \\
& V(x, t)=V_{f}(\rho(x, t)) .
\end{aligned}
$$

Equation (2.6) is known as the fundamental diagram of traffic flow. The resulting non-linear, first-order partial differential equation constitutes the Lighthill, Whitham and Richards (LWR) model

$$
\frac{\partial \rho}{\partial t}+\frac{d Q_{f}(\rho)}{d p} \frac{\partial \rho}{\partial x}=0
$$

or considering the Equation (2.5)

$$
\frac{\partial \rho}{\partial t}+\left(V_{f}+\rho \frac{d V_{f}}{d p}\right) \frac{\partial \rho}{\partial x}=0
$$

Solving Equation (2.9) with the method of characteristics curves (or waves) (Newell, 1993; Leutzbach, 1988), implies that traffic state remains constant along a characteristic curve or wave. At the boundary between two different traffic states a shock waves is formed. propagating with a velocity of

$$
w=\frac{d Q_{f}(\rho)}{d \rho}=\frac{d\left(\rho V_{f}(\rho)\right)}{d p} .
$$



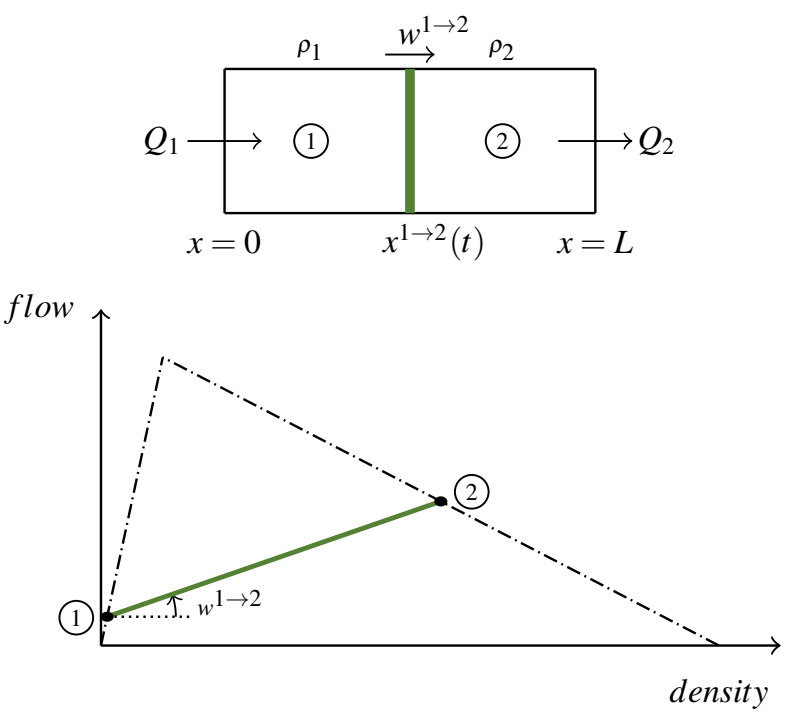

Figure 2.4: A forward moving shock-wave.

In first-order flow models, the characteristic curves are straight lines corresponding to a uniform motion of each region of constant density. Figure 2.4 illustrates the transition from a free flow region, 1 , to a congested one, 2 , for a road section with length $L$. At the instantaneous location of the transition, $x^{1 \rightarrow 2}(t)$, we assume constant flow and densities for both sides. Then, the propagation velocity of a shock-wave that moves between two regions, 1 and 2, associated with different flow $Q_{1}$ and $Q_{2}$ and density $\rho_{1}$ and $\rho_{2}$ respectively is given by

$$
w^{1 \rightarrow 2}=\frac{Q^{2}-Q^{1}}{\rho^{2}-\rho^{1}},
$$

(see Treiber and Kesting, (2013) for the proof of Equation (2.11)).

Danganzo proposed the Cell Transmission Model (CTM) (Daganzo, 1994) which constitutes a time and space discrete numerical solution scheme for the LWR model. The road is divided into homogeneous cells of length $\Delta x$, and for each cell and time step $\Delta t$ a discrete version of Equation (2.8) is solved. Accordingly, the Link Transmission Model (LTM) (Yperman, 2007) is discrete in time, but in contrast to the CTM, the road is divided in homogeneous links, that can be longer than the cells. LTM is based on Newell's simplified kinematic wave theory (Newell, 1993), where the traffic state depends 
only on the boundary conditions of each link, and more specifically on the cumulative flow, $N(x, t)$. Cumulative flow is the number of vehicles that have past the position $x$ by time $t$. Since whole links can be treated at one, the LTM computational efficiency can be higher compared to traditional numerical solution schemes for the LWR model (Nathan Gartner, 2001).

\section{Mesoscopic models}

In macroscopic models, traffic flow is represented as stream with the traffic variables being derived from the analogy to fluid dynamics, while in microscopic models each vehicle follows an individual behaviour. Regarding the level of detail, mesoscopic models describe traffic flow at a medium level, and they can be placed in between microscopic and macroscopic models. Traffic flow can be modelled at an aggregate level, for instance by homogeneous groups of traffic entities. Each one of those groups, is following, though, an individual behaviour. A typical example could be a vehicle platoon with an individual travel behaviour or a lane-change manoeuvre which might be determined by an individual decision, with the decision variables being the macroscopic relative lane densities, and speed differential (Hoogendoorn, 2001). Thus, Mesoscopic models can be applied to larger areas with limited computing and calibration effort, while they provide a better representation of the traffic dynamics and individual travel behaviour compared to the corresponding macroscopic models (Burghout et al., 2006). The three basic families of mesoscopic models used in practice are the so-called headway distribution, cluster, and the gas-kinetic models (Maerivoet and De Moor, 2005).

\subsubsection{Transportation planning models}

Traffic flow models describe the physical propagation of a predefined traffic flow, i.e. number of vehicles per time unit, at a road section. On the other hand, transportation planning models estimate the number of travellers that will choose to travel through this road section. The basic assumptions considered by transportation planning models are that travel demand and supply depend on a number of socio-economic factors and travellers make rational decisions. The transportation planning process, also referred to as four-step model, commonly includes the following steps: trip generation, trip distribu- 
tion, modal split and traffic assignment (Patriksson, 2015; Mcnally, 2000).

The study area is divided in zones, where each zone is assumed to generate and attract trips. During the first step, trip generation, the number of trips originating and terminating in different zones of the study area is determined. Then, transportation networks are assumed to be in equilibrium between the demand, that could be the number of travellers that want to go from an origin to a destination, and the supply, referring to available infrastructure, transport modes or level of congestion. Therefore, considering those aspects, travellers select their destination and time of their trip (trip distribution), the transportation mode to use (modal split) and which way to get there (traffic assignment).

The analysis of the first three steps and the investigation of their effects on emission modelling is outside of the scope of the thesis. The thesis focuses on the last step, traffic assignment. The demand between each origin destination pair, i.e. number of private vehicles that want to go from a specific origin zone to a specific destination zone, is assumed to be known and reliably determined during the previous three steps. Traffic assignment models then assign the demand (static or dynamic) to specific routes of the network, based on some behavioural principals; one principal for instance could be that travellers try to minimize their own travel cost. The basic results of traffic assignment are the traffic volumes and travel times on different roads of the network. One significant assumption considered by the majority of traffic assignment models, is that travellers are experienced, meaning that they are using the traffic network for a sufficiently long period in order to select the most profitable routes.

\section{Static Traffic Assignment}

STA models are commonly used for the strategic assessment of the current or the future efficiency of a transportation network. The traffic demand defined from the previous steps of the four-step model, is static with respect to time, commonly regarding a relatively long period of the day, such as the morning peak period. Thus, the resulting traffic variables, i.e. traffic volume and travel time on each road are also expressed by temporal mean values over the analysis period. The behavioural principle usually considered for the individual route choices is the User Equilibrium (UE). UE is based on Wardrop's first 
principle (Wardrop, 1952) which states that "no driver can unilaterally reduce his/her travel costs by shifting to another route".

Consider the traffic network as a directed graph, $\mathcal{G}=(\mathcal{N}, \mathcal{A})$, which includes a set of nodes $\mathcal{N}$ and a set of links $\mathcal{A}$. Let $\mathcal{R}$ be the set of origin nodes associated with the origin zones, $\mathcal{R} \subseteq \mathcal{N}$, and $\mathcal{S}$ the set of destination nodes concerning the destination zones, $\mathcal{S} \subseteq \mathcal{N}$. Each link of the network, $a \in \mathcal{A}$, is associated with different attributes, such as capacity, $C_{a}$ or speed limit. The desired number of movements from an origin node $r \in \mathcal{R}$ to a destination node $s \in \mathcal{S}$, denoted by $q_{r s}$, is represented by an origin-destination matrix having $|\mathcal{R}|$ number of rows and $|\mathcal{S}|$ number of columns. Furthermore, $\mathcal{K}^{r s}$ is the set of different alternative link sequences, called routes or paths that connect each node $r \in \mathcal{R}$ with a destination node $s \in \mathcal{S}$.

The problem of traffic assignment, then, concerns the way that the Origin-Destination (O-D) matrix will be assigned into the network, namely how the demand will be distributed among the possible paths $k, k \in \mathcal{K}^{r s}$, given that link cost, $c_{a}$, is a function of link flow, $x_{a}$, for each $a \in \mathcal{A}$ (Sheffi, 1985). Each network link, $a$, is associated with a generalized cost, $c_{a}$, which includes all the aspects affecting how onerous the travel is, such as travel time, travel cost, etc. The $\mathrm{UE}$ is a stable condition where all the available paths $k, k \in \mathcal{K}^{r s}$ between the origin $r$ and destination $r$, have the same travel cost and no traveller can improve his or her travel cost by unilaterally changing routes. To find the equilibrium solution in a network, the problem was mathematically formulated as an optimisation problem by Beckmann (Beckmann et al., 1956),

$$
\min z(x)=\sum_{a \in \mathcal{A}} \int_{0}^{x_{a}} c_{a}(\omega) d(\omega),
$$

subject to

$$
\begin{aligned}
& \sum_{k \in \mathcal{K}^{r s}} f_{k}^{r s}=q_{r s} \forall r, s, \\
& f_{k}^{r s} \geq 0 \quad \forall k, r, s,
\end{aligned}
$$

and the definitional constraint

$$
x_{a}=\sum_{r \in \mathcal{R}} \sum_{s \in \mathcal{S}} \sum_{k \in \mathcal{K}^{r s}} f_{k}^{r s} \delta_{a, k}^{r s},
$$

where $f_{k}^{r s}$, is the number of travellers that chose path $k$ between origin $r$ and destination $s$, and $\delta_{a, k}^{r s}$ is the so-called incidence matrix, having 
the value of 1 if link $a$ is on path $k$ between $r$ and $s$, and 0 otherwise. Solving Problem (2.12) will lead to optimal link flows, $x_{a}$, where the travel cost between each O-D pair, $r s$, is the minimum possible and equal for every $k \in \mathcal{K}^{r s}$ with positive flow.

In most of the cases the travel cost, $c_{a}$, is associated with the travel time of each link, $c_{a}=t_{a}$. The link travel time, $t_{a}$, is a function of link flow $x_{a}$, known as the link cost function or Volume Delay Function (VDF). The VDF provides a mathematical relationship between travel time, $t_{a}$ and traffic volume $x_{a}$, representing the measure of the disutility of each corresponding link. Beckmann also proved that Problem (2.12) has a unique solution if

$$
\begin{aligned}
& \frac{\partial c_{a}\left(x_{a}\right)}{\partial x_{b}}=0 \quad \forall a \neq b, \\
& \frac{\partial c_{a}\left(x_{a}\right)}{\partial x_{a}}>0 \quad \forall a .
\end{aligned}
$$

Therefore, according to Equation (2.13), the travel cost of each link should not depend on the flow on other links, and according to Equation (2.14) the VDF should be strictly convex. The latter means that the generalised cost should increase with increasing volume. Specifically, in most of the cases, travel time $t_{a}$ increases strictly with the Volume Capacity Ratio $(\mathrm{V} / \mathrm{C}), x_{a} / C_{a}$. The VDF type named Bureau of Public Roads (BPR, Equation (2.15)) is a commonly used function,

$$
t_{a}\left(x_{a}\right)=t_{0}\left(1+\gamma\left(\frac{x_{a}}{C_{a}}\right)^{\theta}\right),
$$

where $t_{0}$ is free flow travel time and $\gamma, \theta$ are parameters of the VDF. Numerous types of VDFs have been proposed and used in practice including the Davidson's (Davidson, 1966) and the asymmetric Akcelik's function (Akcelik, 1991b), or even more complex functions incorporating delays and queues length (Skabardonis and Dowling, 1997). However, the Problem (2.12) has a unique solution only if the VDF satisfies the conditions 2.13 and 2.14. Several algorithms have been developed to solve the static user equilibrium with the most popular being the Frank Wolfe algorithm (Frank and Wolfe, 1956) and the method of successive averages.

At the simple UE case, the demand of private cars that is subject to assignment. The number of travellers actually chose to travel with 
car instead of public transport, walking etc. has been derived from the previous step of the four-step model, the modal split. An extended version of UE incorporates variable demand (Sheffi, 1985), meaning that the demand of private traffic depends on the resulting minimum route generalised cost for each mode. Considering the generalised cost of the alternative transportation modes, travellers can change their preferred mode. Eventually, the system will reach an equilibrium between the last two steps of the four-step model, where no traveller can improve his/her generalised cost by unilaterally changing routes or transport modes. Due to the relatively simple nature, STA models can be efficiently coupled with demand models, such as discrete choice models.

However, the inherent simplicity of STA can lead to considerable limitations. The classical Beckmann formulation does not explicitly take link capacities into account. In this way, unrealistic flows can be produced, since the assigned volume for a link can be higher than its capacity. However, capacity in static models does not correspond to the maximum flow, it rather represent the saturation flow. By adding simple capacity constraints to the Beckmann formulation, the capacitated (or extended) Beckmann UE formulation is obtained. In Larsson and Patriksson, (1995) a theoretical analysis of the capacitated model is presented and the different types of side constraints and are discussed. Nevertheless, adding these constraints the problem becomes more tedious to solve and more complex algorithms are needed. However, the equilibrium solution can be approximated by the uncapacitated traffic assignment problem using different dual/penalty techniques such as the inner penalty approach and the augmented Lagrangian multiplier that are studied and implemented in Nie et al., (2004). Even though, link flows are more realistic at this extended UE version, route choice could still be unrealistic, since queues and propagation phenomena are not taken into account.

Thus, one of the most significant limitations of STA, which could substantially influence the emission modelling, is associated with the inability of describing flow propagation phenomena. Interactions between links are explicitly not considered, due to condition 2.13 , and therefore the formation and the propagation of the queues are neglected. The latter can have a direct impact to the spatial distribution of emission, affecting all the spatially sensitive applications of emissions discussed in Section 2.1.3. Additionally, in static models, inflow is always equal to the outflow (Chiu et al., 2011), and in principal 
the network loading is instantaneous and the assigned demand determines the steady-state flow and travel time. However, despite the above limitations, STA models fit nicely into the concept of long-term planning, due to low computing cost and low number of parameters for calibration (Maerivoet and De Moor, 2005).

\section{Dynamic Traffic Assignment}

The rationale behind the DTA models is similar to the one considered in STA, namely each traveller in the traffic network follows a certain route based on a behavioural principle. The most significant differences between the two approaches are: i) the demand is not static any more but varies over time and the O-D matrix is time-sliced, ii) the arrival time at a link is different than the departure due to dynamic network loading.

The analysis period is divided in smaller sub-periods, commonly covering some minutes, and the resulting traffic flow and average speed obtain temporal variation. With DTA models being based on the similar behavioural assumptions of individual routing decisions as STA, UE, a major class of DTA problems regards the Dynamic User Equilibrium (DUE). Since, the O-D matrix is time sliced the corresponding DUE can be achieved when all used routes between an origin-destination pair have the same instantaneous or experienced travel time and no unused route has a lower travel time. However, considering the time-varying O-D matrix, the condition of equal travel times, now apply only to travellers who are assumed to depart at the same time.

According to the considered interpretation of travel time, DUE can either be reactive, associated with instantaneous travel times, meaning that traffic conditions in a dynamic network are not predictable, travellers can only get the information on the current states of the network, or predictive, related to the experienced travel time where traffic conditions in a dynamic network are predictable and travellers can get the information on the current and future states of the network. Therefore, regarding the first case, travellers try to minimize their individual travel time by continuously updating their route choices according to the current states of the network. Contrariwise, in the case of a predictive DUE, travellers make their choices considering the information of the future state of the network and the predicted travel time. 
Solving the DTA problem becomes more complex than the static one, and usually the solution algorithms iterate between the route choice and the dynamic network loading, increasing the computing time and memory requirements. At every iteration of the algorithm, a route choice is performed where according to the predefined travel times from the previous iteration and depending on the optimisation algorithm, a part of the demand $q_{r s}$ is assigned into different paths $k \in \mathcal{K}^{r s}$, determining new inflow rates for each link $a$. Next during the network loading phase, the inflow is propagated based on traffic flow models, resulting into new link travel times, $t_{a}$, to be subject of the next iteration's route choice. Then, this procedure is repeated until some convergence criteria, that could regard for instance the travel time of the alternative routes between an O-D difference, are satisfied. Tong and Wong, (2000) provide a solution for the predictive DUE using an iterative algorithm that is based the method of successive averages.

The different approaches of dynamic network loading proposed in the literature can be classified into: simulation based and analytical link-based approaches (Peeta and Ziliaskopoulos, 2001). On one hand, simulation approaches being based on microscopic or mesoscopic traffic flow models, are more realistic but they can be computationally extensive (Nie and Zhang, 2005). On the other hand, analytical approaches consider macroscopic traffic flow variables, and hence can be more efficient. The analytical approaches can either regard mathematical formulations including the work of Merchant and Nemhauser, (1978) and of Janson, (1991) where a heuristic approach is employed to solve the predictive DUE, or optimal control formulations (Friesz et al., 1989). Traffic flow propagation during the dynamic network modelling can also be based on the macroscopic traffic flow models, described in Section 2.2.1, such as the CTM (Ziliaskopoulos, 2000) or the LTM (Gentile, 2015; Yperman, 2007; Raadsen et al., 2016).

One significant mechanism incorporated in models such as the LTM and CTM is the spill-back when inflow exceeds capacity. Figure 2.5 illustrates the way that LTM propagates shock-waves into a network. Consider two simple consecutive links $j$ and $j+1$, where the inflow of link $j, Q_{j}^{1}$, is greater than the capacity of link $j+1$, $C_{j+1}$. Because link $j+1$ cannot receive flow greater than its capacity, the actual flow that will be transmitted from link $j$ to link $j+1, Q_{j}^{2}$, equals to the capacity of link $j+1, C_{j+1}$. LTM blocks the link's up- 

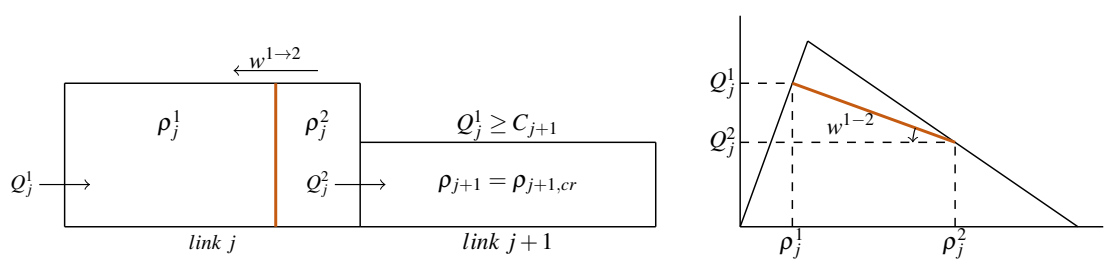

Figure 2.5: Backwards moving shock-wave created by reduced capacity on link $j+1$.

stream boundary up to link's capacity, by applying a node model that is associated with node capacity, sending and receiving flows, similar to the supply-demand method used in CTM. Therefore, $C^{j+1} \Delta t$ vehicles are actually transmitted during a time period $\Delta t$ from link $j$ to link $j+1$, and the rest vehicles $\left(Q_{\alpha}^{1}-C_{j+1}\right) \Delta t$, form a queue (state 2 ), with higher density $\rho_{j}^{2}>\rho_{j}^{1}$. The density at the state 1 is, according to the inverse of the fundamental diagram, $\rho_{j}^{1}=\rho_{j}\left(Q_{j}^{1} \mid\right.$ uncongested $)$ (if we assume that $Q_{j}^{1}<C_{j}$ ) and the speed $V_{j}^{1}=Q_{j}^{1} / \rho_{j}^{1}$, while the state 2 is associated with a flow $Q_{j}^{2}$, a density $\rho_{j}^{2}=\rho^{j}\left(Q_{j}^{2} \mid\right.$ congested $)$ and a speed $V_{j}^{2}=Q_{j}^{2} / \rho_{j}^{2}$. The state 2 is propagating through the link $j$ (see Equation (2.11) with a speed $w^{1 \rightarrow 2}$

$$
w^{1 \rightarrow 2}=\frac{Q_{j}^{2}-Q_{j}^{1}}{\rho_{j}^{2}-\rho_{j}^{1}} .
$$

Figure 2.5 illustrates a very simple network, where no traffic is merged or diverged. However, realistic networks are more complicated, nodes represent merges, diverges or intersections, and therefore two or more links can enter or/and leave one single node. LTM algorithm includes a node model, in which the splitting probability is demand proportional and vehicles are served in a First In First Out (FIFO) sequence, similar to the CTM's merging and diverging model (Daganzo, 1995).

Nevertheless, in CTM and LTM, time is discretised and Equation (2.8) is solved at every time step. Hence, for short discretisation intervals, high computing times discourages their usage for large networks. The time discretisation can be eliminated by the event-based Link Transmission Model (eLTM) algorithm proposed in Raadsen et al., (2016). According to the event-based method, the simulation step is updated only when an event occurs. There are three possible types of events, when a backward shock-wave hits the most upstream end of 
a link, when a forward meets the downstream end, and finally when a shock-wave meets another one somewhere along the link. Thus, the number of time steps is significantly decreased, reducing the computing time, as well as the required memory.

Concluding this section, DTA models can be considered as more suitable than STA to generate input data for emission models since they result in a higher resolution of time average flows and speeds and provide techniques for realistic flow propagation. However, despite the intensive research during the last years, DTA still remains a challenging issue in traffic modelling (Gentile, 2015). In general, a unique equilibrium solution does not exist, which may create difficulties during the comparison of different policy measures. Additionally, DTA heavily rely on the availability of high resolution data, both in terms of demand and calibration. Furthermore, when DTA are coupled with demand models, the complexity is increased in comparison to the corresponding STA case. In addition to the above limitations, the extensive computing effort needed, discourage the use of DTA in larger areas.

\section{Quasi-Dynamic Traffic Assignment}

Quasi-dynamic models can be considered as a bridge between STA and DTA since they are placed in the middle of these two approaches both in terms of computing efficiency and reliability. While demand between each O-D, $q_{r s}$, is usually constant during the analysis period, network loading is quasi-dynamic. The distribution of the demand, $q_{r s}$, along the paths $k, k \in \mathcal{K}^{r s}$ can be computed solving Problem (2.12) similarly to STA. Assume that $\mathcal{D}^{r s, k}$ is the set containing the consecutive links of path $k, k \in \mathcal{K}^{r s}$. The term quasi-dynamic means that part of the demand $f_{k}^{r s}$ is loaded in the path $k$ instantaneously, similarly to the static case, until a link flow $x_{a}, a \in \mathcal{D}^{r s, k}$, reaches its capacity $C_{a}$. Then, path $k$ is blocked at the upstream end of the link and the rest of the demand $f_{k}^{r s}$ is forming a queue propagating upstream of link, a, dynamically. Real time variable doesn't exist, but the propagation of the queues is based on pseudo-time from the time that the flow of link $a$ reaches the capacity $C_{a}$.

The most characteristic models belonging in this family are suggested by Bundschuh et al., (2006a) and Bliemer et al., (2012). Regarding the first model, the resulting flows, $f_{k}^{r s}$, after the STA problem is solved, are incrementally loaded until the capacity of a link $C_{a}$, 
$a \in \mathcal{D}^{r s, k}$ is reached. Then, queues are propagated backwards relying on the queuing capacity of each link, upstream the link $a$. When the whole demand has been fed into the network, the traffic stored in the queues is propagated forward and queues are dissolved in small time slices, until the network become empty. The final link delays are computed depending on the congestion distribution among the above time slices.

The rationale behind the model proposed by Bliemer et al., (2012), Static Traffic Assignment with Queuing (STAQ), is similar to the one of Bundschuh et al., (2006a), with the basic difference being that queue propagation is more realistic since it is based on the LWR theory. STAQ actually relies on the eLTM model, considering two static assumptions: the demand is stationary over a given period and traffic propagates instantaneously.

The model consists of two phases, the squeezing and the queuing phase. During the squeezing phase, in which there is no time variable, the predefined demand from STA, of each path $k, f_{k}^{r s}$, is loaded into the network through a more efficient compared to Bundschuh et al., (2006a), incremental assignment which ensures that no link flow $x_{a}, a \in \mathcal{D}^{r s, k}$, exceeds capacity. When the capacity of link $a$, $a \in \mathcal{D}^{r s, k}$, is reached, the remaining demand forms a vertical queue at the upstream end of link $a$. Next, during the queuing phase the effect of spill-back is considered, and queues propagate to the upstream links. The propagation of the queues is determined by the shockwaves speeds calculated according to Equation (2.16), while the node model is similar to one employed by LTM . The queuing phase ends when all traffic demand has reached its destination. The average link travel time can then be calculated in terms of delays obtained from the cumulative inflows and outflows.

For both models the route choice is considered as exogenous. Using the same iterative algorithms as in the case of DTA, the travel times of each path between an $\mathrm{O}-\mathrm{D}, k, k \in \mathcal{K}^{r s}$, can be equilibrated. Nevertheless, the condition 2.13 is not satisfied due to spill-back, and hence the uniqueness of the solution is not ensured. Quasi-dynamic assignments are computationally more efficient than DTA, but since link interactions are considered, similarly to the DTA case, concerns are raised regarding the uniqueness of the equilibrium solution. A non-unique solution can potentially lead to a problematic evaluation of different alternative policies. However, using quasi-dynamic loading as post-processing of STA results, can improve applications such 
as emission modelling, since the location of the congestion is more realistically described. In Chapter 5 of this thesis, a version of the STAQ model, suitable for emission estimation, is proposed and its application is evaluated.

\subsection{Traffic measurements}

For a more reliable estimation of the real traffic conditions, traffic models are commonly used in combination with traffic measurements. Traffic measurements are used for calibration and validation of traffic models, for real-time traffic control or for the direct determination of the traffic state. The basic types of traffic measurements and the methods of collecting the traffic measurements data are described in Section 2.3.1, while Section 2.3.2 presents some methods for estimating the traffic state from measurements.

\subsubsection{Measurements types and devices}

Most of the existing devices for measuring traffic are fixed-point sensors, collecting Eulerian or cross-sectional data. Cross-sectional data measured from stationary sensors usually is expressed by temporal mean quantities related to the macroscopic traffic flow variables, such as traffic flow, mean speeds or occupancy for a specific location. Stationary sensors cannot directly measure traffic density since it is a spatial defined quantity (Treiber and Kesting, 2013). Nevertheless, traffic density can be estimated using, for instance, the hydrodynamic relationship of Equation (2.4).

The most commonly used devices for collecting cross-sectional data, are the induction loop detectors and the radar sensors, being installed in the major streets of many European cities. Loop detectors are the most widespread sensors, which sense the presence of a conductive metal object by inducing currents in the object while the loop inductance is reduced (Neudorff et al., 2003). The most important disadvantages associated with the use of loop detectors are the vulnerability and the installation and maintenance cost. Since they are installed in the roadway surface, for any maintenance traffic is interrupted. On the other hand, radar sensors, being usually installed on bridges above the traffic can be maintained without traffic interruption. They transmit energy and a portion of this energy is 
scattered by the vehicle back toward the sensor, where it is detected and converted into traffic information (Neudorff et al., 2003). Radar sensors are strongly influenced by temperature and they also become inadequate for high congestion levels when speeds are very low or close to zero. Other devices usually used in practice for collecting cross-sectional are the light-based and the magnetic sensors.

During the last decades, due to the extensive research on the field of Intelligent Transportation Systems (ITS), Lagrangian measurements are also available, providing microscopic, trajectory data. Trajectory data refers to the space-time location of each individual vehicle-driver unit and it can be considered as one of the most comprehensive traffic data available (Treiber and Kesting, 2013). Microscopic variables, such as individual location, speed and acceleration can be directly or indirectly estimated from a trajectory, while macroscopic variables can also be estimated from a trajectory data set, including all vehicles within a given road segment and period. A trajectory data set, can be obtained from camera-based methods, where video image processing techniques are employed to estimate the position of each vehicle in time from video footages. The algorithms, however, needed for these methods have to be robust, increasing the cost while luminosity and visibility changes can affect the accuracy of the measurements.

Global Positioning Systems (GPS) equipped devices are an alternative source of Lagrangian measurements. The corresponding collected traffic data, is also called Floating Car Data (FCD). FCD can be obtained from different probe client types, such as navigation systems, fleet management clients and insurance black-box systems. Other methods of collecting floating-car data are associated with cellular networks and Automated Vehicle Identification (AVI), such as license plate recognition and re-identification of Bluetooth or Wi-Fi physical address. An overview of traffic sensors and floating-data collection techniques can be found in (Allström et al., 2017). Since floating-car data regards only a few probe vehicles, some macroscopic traffic variables essential from an emission modelling point of view, such as traffic flow or average speed can be estimated only under extensive assumptions. 
(a)

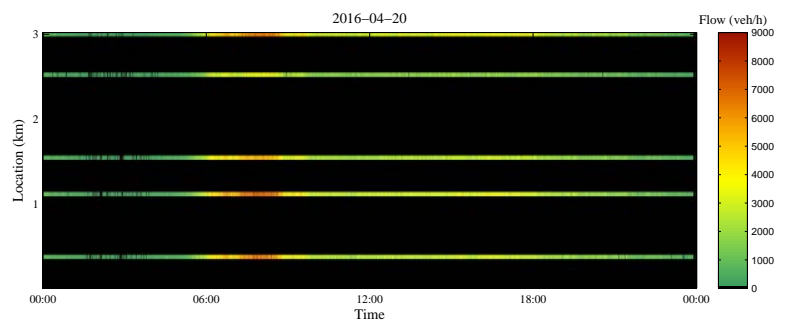

(b)

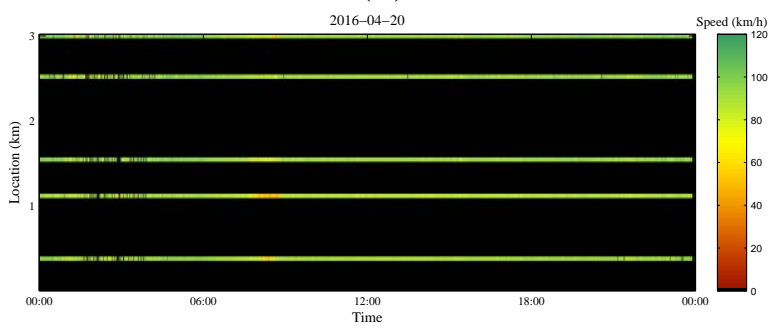

Figure 2.6: Cross-sectional data from radar sensors installed on a motorway stretch in Stockholm; (a) flow (b) speed.

\subsubsection{Traffic state estimation}

The term traffic state estimator refers to the process of the inference of traffic state variables (traffic flow, traffic density, speed) on road segments using partially observed traffic data (Seo et al., 2017). Since it is not financially feasible to equip every part of a road network with sensors, the collected traffic data usually contains gaps in space and time. Figure 2.6 is an illustrative example of the missing information. It depicts the output of 5 radar sensors installed on a $3 \mathrm{~km}$ long motorway segment in Stockholm, for a typical day in 2016, plotted on the space-time domain. The nature of the data collected by radar sensors is cross-sectional, and hence the measured values regard only specific locations, while there is no indication of the traffic conditions in between two sensors. In addition, we can notice some time gaps in the data because possibly the sensors were temporally out of order. Thus, the main aim of an estimator is to reconstruct the space-time field as well as to remove the measurements' noise, providing more reliable and comprehensive information about the traffic state. Traffic state estimators are a useful tool for applications such as traffic management and control. 
The two basic components of a traffic state estimator are the priori knowledge of traffic and the partial observations. Depending on the type of the prior knowledge that are based on, Seo et al., (2017) categorise the state estimating methods into three groups: model-driven, data-driven and streaming-data-driven. The approaches belonging into the first category, employ traffic flow models for the state estimation. The data-driven approaches depend on historical data and rely on statistical methods, while the third category combines the two previous elements. Partial observations can regard data from one but as well as from more than one heterogeneous data sources; the procedure of combining this data is called data fusion.

The level of detail, the complexity, as well as the aggregation level of the output traffic parameters varies among the different state estimation approaches, depending mainly on their potential application. For large scale applications, such as emission estimation on a city level, usually data-driven, non-parametric methods, such as AADT, are applied for estimating the required missing data.

\section{Estimation of annual average daily traffic}

The basic aim of the AADT approach is to estimate the traffic state and traffic activity for each day or hour of the year and for network parts where there are not permanent measurement data available. Each such part of a network, is assigned into a road group based on short-term measurements. Then, seasonal factors, computed from permanent measurements at different network locations, are applied over an estimated annual average daily traffic flow.

Based on data from permanently installed sensors, here referred as Permanent Traffic Counters (PTC), a time (day/season) variation factor can be computed, describing the average daily flow for a specific type of days and road segment in proportion to the AADT. This factor can include characteristics related to specific type of days (e.g. weekdays and weekends) and to seasons (e.g. months). However, since PTCs can be installed only at some sites of the traffic network, we assume that short period traffic counts (Short Period Traffic Counts (SPTC)) are commonly available for the rest of the network, providing data for shorter time periods (e.g. weeks or months). The AADT for every SPTC site, is then estimated based on the time variation factor derived from a PTC located in the same road category.

For every PTC site, $\alpha$, the AADT can easily be computed, just 
by finding the mean of the annual daily flow. A key issue is then to group the days of the year into clusters, and group PTCs into road categories, for which we expect the time variation factor to be similar. This can be done using methods such as the k-means clustering (Hartigan and Wong, 1979) or fuzzy C-means clustering (Bezdek et al., 1984). A common grouping of days is to cluster based on day of week and month of the year. Let $I$ denote the set of road categories, and for a specific road category group $i \in I, C_{i}$ denotes the set of sites belonging to this category. Let $J$ be the set of day clusters. For road category, $i \in I$, and day cluster, $j \in J$, the time variation factor can be computed as

$$
f_{i, j}=\frac{1}{\left|C_{i}\right|} \sum_{\alpha \in C_{i}} \frac{q_{j, \alpha}^{A D T}}{q_{\alpha}^{A A D T}},
$$

where $q_{j, \alpha}^{A D T}$ is the average daily flow at site s for day cluster $j$, and $q_{\alpha}^{A A D T}$ is the annual average daily flow at site $s$.

The next step is to assign the SPTC sites, to one of the road categories and estimate their average annually flow. This can for instance be done using artificial neural networks (Gastaldi et al., 2013) or regression models (Zhong et al., 2004). Once a SPTC site, $\beta$, is assigned into a road category group $i$ and the average annually flow, $q_{\beta}^{A A D T}$, is defined, the average daily flow for the day cluster $\mathrm{j}$ can be estimated as

$$
q_{j, \beta}^{A D T}=q_{\beta}^{A A D T} \cdot f_{i, j}
$$

Depending on the use of the AADT, it may be necessary to also include an hourly factor. Emissions are sensitive to temporal traffic variation, and as highlighted in Batterman et al., (2014), they should be estimated in a daily and in an hourly level. As discussed in Tsanakas et al., (2017), one hour is a sufficiently short period to capture the effects of flow temporal variations in an emission estimation analysis. The hourly pattern can also be determined from the PTC sites, and can be included in the clustering analysis (Ha and Oh, 2014) and in the estimation procedure as proposed in Gastaldi et al., (2013). Therefore, for every road category $i$, and day cluster $j$, the hourly factor can be computed as

$$
g_{i, j, h}=\frac{1}{\left|C_{i}\right|} \sum_{\alpha \in C_{i}} \frac{q_{j, h, \alpha}^{H T}}{q_{j, \alpha}^{A D T}},
$$


where $q_{j, h, \alpha}^{H T}$ is the hourly average flow for hour $h$, on day cluster $j$, at site $\alpha$. Using the hourly factor together with estimated AADT and time variation factor, hourly average flows can be computed for all SPTC sites:

$$
q_{j, h, \beta}^{H T}=q_{j, \beta}^{A D T} \cdot g_{i, j, h}
$$

However, for a comprehensive emission estimation, together with the flow, the temporal variation of speeds should also be included. If we assume that the same type of data as flow from the PTC sites is available also for the average speed, following the same methodology, the corresponding Annual Average Daily Speed (AADS) can be calculated. The final output of the method is a coherent set of hourly average speeds $V_{j, h}^{H T}$ and flows $Q_{j, h}^{H T}$, for every $h$ hour of the $j$ day of the year.

\section{Spatio-temporal interpolation}

A more sophisticated, streaming-data-driven traffic state estimation approach, which is based on spatio-temporal interpolation and smoothing techniques, is suggested in Treiber and Helbing, (2002) and Treiber and Kesting, (2013). The ASM, fully reconstructs the measured speed and flow field, by both estimating temporally missing data and filling the spatial gaps between stationary sensors. The output of the interpolation are a complete speed $V(\kappa, \tau)$ and flow $Q(\kappa, \tau)$ fields as a function of the discretised space and time intervals $\kappa, \tau$. In practise there is a need to discretise, because of both computational burden and emission modelling issues. Regarding the latter, an macroscopic emission model should be applied on stable traffic conditions, for which the driving cycles can be expected to be valid. For this reason we introduce the $V_{\kappa, \tau}$ and $Q_{\kappa, \tau}$ which denote the discretised speed and flow measurements.

The interpolation method relies on a discrete convolution, with a symmetric exponential function as the weighted kernel, $\phi_{0}$, operating actually as a low-pass filter, smoothing temporal variation and spatial fluctuations. More specifically, the method considers that all the traffic perturbations are propagating either downstream, in free traffic conditions, with a wave speed $c_{f r e e}$, or upstream, in congested conditions, with a wave speed $c_{\text {con }}$. Assume that $z\left(\kappa_{i}, \tau_{j}\right)$, is the value of the $z$ traffic variable (in our case speed and flow) counted by the stationary sensor $i$, at position $\kappa_{i}$ and time $\tau_{j}$, where $j$ denotes the 
the index of the aggregation time intervals. At each position $\kappa$ and time $\tau$, two linear anisotropic low-pass filters $z_{\text {free }}$ and $z_{\text {con }}$ can be computed as

$$
z_{\text {free }}(\kappa, \tau)=\frac{\sum_{i} \sum_{j} \phi_{0}\left(\kappa_{j}-\kappa, \tau_{j}-\tau-\frac{\kappa-\kappa_{i}}{c_{\text {free }}}\right) \cdot z\left(\kappa_{i}, \tau_{j}\right)}{\sum_{i} \sum_{j} \phi_{0}\left(\kappa_{j}-\kappa, \tau_{j}-\tau-\frac{\kappa-\kappa_{i}}{c_{\text {free }}}\right)}
$$

and

$$
z_{\text {con }}(\kappa, \tau)=\frac{\sum_{i} \sum_{j} \phi_{0}\left(\kappa_{j}-\kappa, \tau_{j}-\tau-\frac{\kappa-\kappa_{i}}{c_{c o n}}\right) \cdot z\left(\kappa_{i}, \tau_{j}\right)}{\sum_{i} \sum_{j} \phi_{0}\left(\kappa_{j}-\kappa, \tau_{j}-t-\frac{\kappa-\kappa_{i}}{c_{c o n}}\right)},
$$

The Kernel $\phi_{0}$ is given by

$$
\phi_{0}(\kappa, \tau)=\exp \left(\frac{|\kappa|}{\sigma}-\frac{|\tau|}{\psi}\right)
$$

where $\sigma$ and $\psi$ are the spatial and temporal smoothing widths. Finally, the estimated traffic variable constitutes a superposition of the two low-pass filters

$$
z(\kappa, \tau)=w(\kappa, \tau) z_{\text {con }}(\kappa, t)+(1-w(\kappa, \tau)) z_{\text {free }}(\kappa, \tau),
$$

with $w(\kappa, \tau)$ being a weight factor $0 \leq w \leq 1$. 



\section{Chapter 3}

\section{Emission estimation based on traffic measurements}

The traffic data required as input to emission models can either be derived from traffic models or traffic measurements. This chapter focuses on the use of traffic data that comes from measurements, and specifically on the use of cross-sectional data from stationary detectors in an emission estimation analysis. Section 3.1 provides an overview of the approaches commonly used to estimate emissions from traffic measurements including both cross-sectional and trajectory data. In the same section we also explain our choice to use cross-sectional data, since stationary detectors still remains a cost-efficient method of collecting traffic data to be used in emission modelling. Next, in Section 3.2 the main problems related to the use of cross-sectional data in emission modelling are described. Those issues are mainly associated with the simplified traditional approaches of extrapolating the traffic data in time and space. For this reason, we suggest the use of a more sophisticated extrapolation method and we evaluate its application compared to the traditional approaches following the methodology described in Section 3.3. Section 3.4 presents the results while Section 3.5 concludes and discusses the basic findings of this chapter. 


\subsection{Review of the methods}

Traditionally, macroscopic emission models, rely on outputs from the transportation planning models based on STA. However, many studies (Aguilera and Lebacque, 2010; Bai et al., 2007a,b) have highlighted the difficulty of using such data as input to emission modelling, since the modelling of traffic dynamics is limited. Therefore, many researchers have moved towards the exploitation of the increasingly accessible data from traffic sensors (see Section 2.3), either to be used in combination with traffic models, or on its own as the basis for computing emissions.

\subsubsection{Approaches based on trajectory data}

Traditionally, traffic measurements were mainly available from fixed sensors, but today there might also be additional sources of data, such as data from vehicle probes. It is important to note that new sources of traffic data, such as AVI or GPS, mainly contribute to the collection of speeds or travel times, while for the traffic volumes additional sources of data are needed.

In Gately et al., (2017), emissions are estimated by assimilating GPS data with speed and flow data derived from stationary detectors or a traffic model. Nyhan et al., (2016) use GPS trajectory data from taxis, available for a large urban area in Singapore, to estimate emissions. However, they conclude that Singapore is a special case, where taxi data can be used to infer general traffic pattern and the total volume, but this is not necessarily true for other urban areas. Jing et al., (2016) estimate emissions for Beijing based on hourly average segment speed form GPS floating car data. The flow is derived from average speed using a functional relationship between speed and flow. Nevertheless, the floating car data covered only two weeks of data for the entire city. In Ryu et al., (2015), GPS data is used to obtain speeds as input to the emission calculation, but only for specific vehicles and network routes.

Therefore, although trajectory data is becoming more and more accessible, it is not always possible to have a complete set of both speeds and flows. Additionally, these approaches can be expensive, require significant participation of the drivers and have privacy concerns (Jeng et al., 2013). 


\subsubsection{Approaches based on cross-sectional data}

On the other hand, cross-sectional data, from stationary detectors (inductive loop detectors or radar sensors), are able to more efficiently provide comprehensive data for an emission estimation analysis. However, they only provide this data for specific points in the traffic network, and there is no information on what is happening in between two detectors. An example of the use of cross-sectional data for emission modelling purposes is given by Jeng et al., (2013), where a methodology for estimating emissions using inductive loop signature data to derive flow, space-mean speed and vehicle fleet composition is proposed.

Nevertheless, even in the case of stationary detectors, a complete, with respect to time and space, data set, is not always economically feasible. For a reliable emission inventory, the available measured data should be extended in time and space using methods for missing data imputation. A common approach to estimate the traffic activity on a road with missing data or without permanent counting station, is to estimate the annual average daily traffic flow AADT and then multiply it by seasonal or hourly variation factors (see Section 2.3.2). The seasonal curves and the hourly traffic profiles can be computed with the help of permanent counting stations located in different parts of the network. The roads where those permanent stations are located are then divided in categories. Each road with missing data, is assigned to the appropriate road category based on short-period measurements, usually using regression models or neural networks, and the corresponding seasonal curves are applied. A detailed review of the traditional methods for estimating missing counts can be found in Zhong et al., (2004). Commonly, only the traffic activity is estimated through AADT, and the traffic state (usually represented by the average speed) is derived from other sources or through some analytical relationship between speed and flow. There are also examples where flow and capacity ratios are used (e.g. Trafikverket, 2012), to directly determine emission factors. These approaches, however, introduce the problem that there is not a one-to-one mapping between average speed and flows, and the same flow can be measured both at an uncongested (high speed) and a congested (low speed) traffic state.

To improve the accuracy of emission estimations, Fu et al., (2017) attempt to extend short-period data to AADT using neural networks. 
Accordingly, Kholod et al., (2016) derive AADT from video based detection at several locations and types of roads, while Coelho et al., (2014) use manual traffic counts to estimate AADT. In each one of these three studies, GPS data is used to obtain the average speed on each road segment or road category. Alternatively, Lindhjem et al., (2012) use cross-sectional data to develop temporal allocation profiles for the traffic flow, and speed is derived from flow, using the Bureau of Public Roads function (BPR) travel time function. In many other recent studies (Batterman et al., 2014, 2015; Basarić et al., 2014), the estimation of emissions is based on AADT.

\subsection{Limitations associated with cross-sectional data}

For a comprehensive emissions analysis, the ideal situation would be to install permanent traffic sensors on every road segment in the network, obtaining faultless traffic data, 365-days around the year. However, due to limitations such as costs related to installation and maintenance, this is not feasible and real data sets usually contain a significant percentage of missing data. The required data associated with the parts of the network where there are no permanent traffic sensors, usually is estimated by approaches such as AADT. With respect to emission modelling, there are two main limitations with using AADT: (1) average conditions do not capture the most congested days or hours, and (2) applying air quality analysis based on average traffic conditions but with specific background pollution and meteorology data risks to introduce errors in the actual concentration of pollutants. Moreover, all the studies mentioned in the previous section, focus more on the seasonal, or within day variations of flow, while average speed is considered as temporally constant. Even though, temporally constant average speed may not affect applications of emissions modelling such as NEI, speed variations is a significant factor with regard to dispersion or exposure modelling.

Additionally, cross-sectional measurements are by their nature discrete in space, and by taking the spatial average of such measurement along a homogeneous segment (Ferm and Sjöberg, 2015, Muller-Perriand, 2014), the spatial variations of speed and flow are neglected. Despite the fact that average segment speeds can predict travel times sufficiently accurate, their non-linear relationship to 
emissions could lead to considerable errors in an emission estimation analysis.

\subsection{Comparison of the traffic estimation methods}

Considering the limitations related to the use of the traditional data imputation approaches such as the AADT described above, our hypothesis is that the use of a more detailed traffic state estimator, will lead to reliable total yearly emissions and distribution of those emissions in time and space. In order to investigate this hypothesis, we calculate emissions for a road stretch in Stockholm and for the whole 2016 considering two different methods for obtaining the traffic data. The first method relies on the traditional estimation approaches while the second is based on the ASM, corresponding to the traffic data set $i$ and traffic data set ii accordingly in Figure 1.3. By evaluating the differences between the resulting emissions derived from the two different approaches (emission data set $i$ and emission data set $i i$ ), both from a NEI and from air-quality perspective, considering also the efficiency of each approach, we give answer to the research Q1 of this thesis.

\section{Introduction to the study area}

Stockholm, due to the rapidly increasing population, is one of the most congested cities in Western Europe. Local and national road authorities try to alleviate congestion and decrease the travel times, both at a strategical planning level, by establishing a congestion charging scheme, and at an operational management level, by ramp-metering, variable-speed-limits or any other local setting. However, for the efficient application of any congestion mitigation strategy, real measured data for feedback or calibration is needed. For this reason, Swedish Transport Administration collects traffic data, mainly from fixed sensors but also from probe vehicles.

There are approximately 1500 fixed detectors, located all around Stockholm, installed on motorways and arterials (Figure 3.1). Almost two third of them are radar sensors, located at the E4 motorway, being an integral part of a Motorway Control System on the main highway that passes through Stockholm (Allström et al., 2011). 


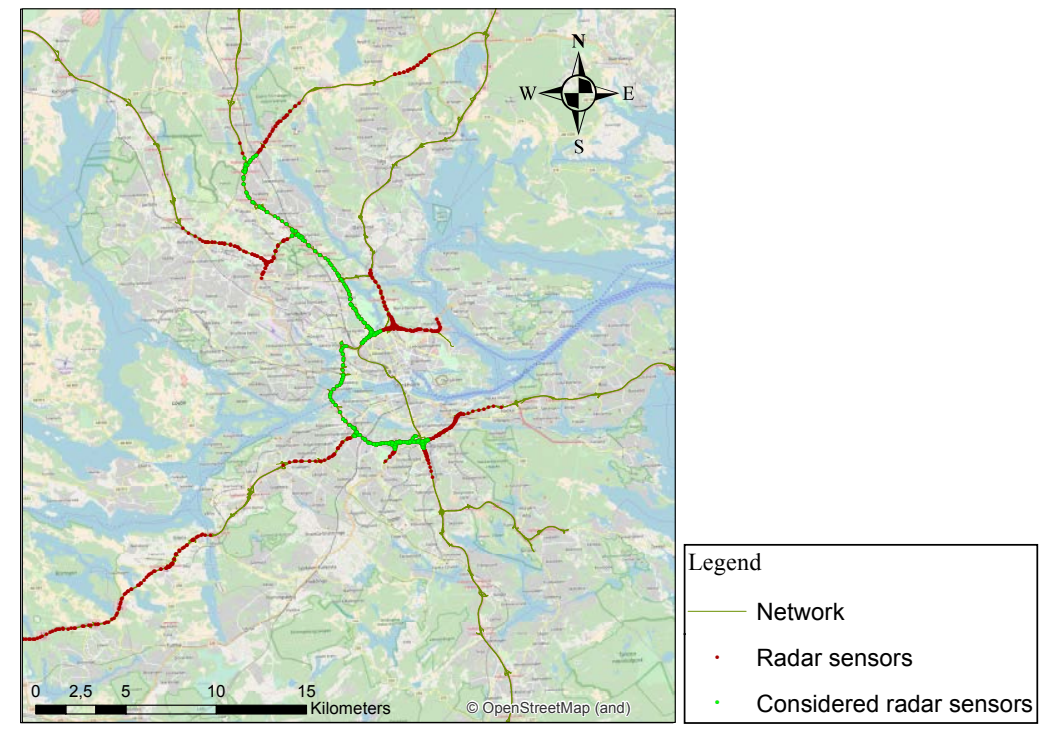

Figure 3.1: Modelled motorway section and the sensors' position.

Our case-study segment is a $23.4 \mathrm{~km}$ long section, which is part of the E4 (Figure 3.1). The segment consists of 83 network links, which are grouped, based on geographical and other characteristics such as the number of lanes, into 19 homogeneous road segments. Moreover, 92 detector stations, are located every 250-300 metres along this segment of the motorway. Each detector station includes a number of radar sensors equal to the number of lanes at the location which collect one-minute average time mean speed and traffic flow data. Here we use the mean flows and speeds across all lanes, from the first of January 2016 to the last of December the same year.

\section{Emission model}

In this study we use the HBEFA emission factor described in Section 2.1.2 adapted for the Swedish roads and traffic conditions. Using information of the Swedish vehicle fleet composition (see Appendix A, Table A.1), we calculated the weighted average of the emission factors corresponding to different vehicle classes, fleet mix and mileage. Finally, our estimations are based on emission factors for five pollutants ( $\mathrm{HC}, \mathrm{CO}, \mathrm{NO}_{\mathrm{x}}, \mathrm{CO}_{2}$ and $\mathrm{PM}$ ), for four traffic situations (Free flow; Heavy; Saturated; Stop and go) and for two different road 
Table 3.1: Volume/Capacity ratio thresholds for the traffic situation determination suggested by the Swedish Traffic Agency Handbook for road traffic and air pollution (Trafikverket, 2012). Each row is associated with a different road type indicated by the speed limit.

\begin{tabular}{lllll}
\hline $\begin{array}{l}\text { Speed } \\
\text { limit }(k m / h)\end{array}$ & $\begin{array}{l}\text { Free } \\
\text { flow }\end{array}$ & Heavy & Saturated & $\begin{array}{l}\text { Stop } \\
\text { and go }\end{array}$ \\
\hline 90 & $<0.65$ & $0.65-0.85$ & $0.85-1.35$ & $\geq 1.35$ \\
70 & $<0.39$ & $0.39-0.84$ & $0.84-1.35$ & $\geq 1.35$ \\
$<50$ & $<0.52$ & $0.52-0.78$ & $0.78-1.22$ & $\geq 1.22$ \\
\hline
\end{tabular}

types (urban motorway (URB/MW) with a speed limit of 90 and 70 $\mathrm{km} / \mathrm{h}$ ). We should highlight here that we consider only exhaust hot emissions and only light duty vehicles. An example of the $\mathrm{CO}_{2}$ and $\mathrm{NO}_{\mathrm{x}}$ HBEFA emission factors that reflect the Swedish conditions is illustrated in Figure 2.3, while Table A.2 in Appendix A contains the values of the corresponding emission factors for every considered pollutant.

The only existing official description (Trafikverket, 2012), of how the traffic situation in HBEFA should be determined for road in Sweden, is based on the $\mathrm{V} / \mathrm{C}$ ratio of each network link (Table 3.1). This is relevant when applying HBEFA with outputs from a STA model, where flow can exceed capacity. Static assignment models allow the volume to be higher than the capacity of a road since the underlying VDF represents travel times as a function of demand rather than the actual flow. However, in reality it is not possible for the flow to be higher than the capacity and accordingly, by applying the $\mathrm{V} / \mathrm{C}$ thresholds on measurements the Stop and go situation cannot occur. However, as it is depicted in Figure 2.3, the Stop and go situation corresponds to the higher emission factor, and hence the accurate prediction of this situation becomes essential.

Therefore, the V/C thresholds, presented in Table 3.1 cannot directly be applied to the measurements. For this reason, we transform the $\mathrm{V} / \mathrm{C}$ thresholds into speed thresholds using the fundamental diagram of traffic flow. In contrast to the VDF, according to the fundamental diagram the relationship between flow and speed is not monotonic. Low flows can correspond either to low speeds in the case of saturated conditions, or to high speeds in the case of non-saturated 
conditions. Since flow cannot by itself determine the traffic situation, we converted the $\mathrm{V} / \mathrm{C}$ ratio thresholds into speed thresholds. Using linear-hyperbolic fundamental diagram, proposed by Work et al., (2010), the flow thresholds are projected to the speed axis in order to determine the speed thresholds, as illustrated in Figure 3.2 (a) Therefore, HBEFA emission factors are expressed as a discrete function, $\tilde{e}^{p}(v)$, of average speed $v$ for each pollutant $p$.

In order to avoid large changes between the emissions factors for small changes in the average speed, we suggest a continuous version of HBEFA derived by linear interpolation between the emission factors corresponding to adjacent traffic situations (Figure $3.2(\mathrm{~b})$ ), similar to the interpolation done in Wismans et al., (2013). Finally, the emission factor is expressed as a continuous function, $e^{p}(v)$, of average speed $v$ for every pollutant $p$.

As mentioned in Section 3.3, each radar sensor collects one-minute mean speed and traffic flow data, which can be used as input for HBEFA emission factors determination. However, the nature of the HBEFA emission factors are not instantaneous, they rather represent an aggregate situation, relying on average conditions during a driving cycle. Hence, the temporal interval of one minute, may not be a sufficiently long interval to describe a HBEFA traffic situation. For this reason, we aggregate the sensors outputs, speed and flow, temporally over an hour. The temporal interval of one hour is more representative for the HBEFA traffic situations while at the same time the speed variations and the demand fluctuations can be sufficiently caught (Tsanakas et al., 2017). After the aggregation of the data, the average hourly data availability for the 92 sensors and the for whole 2016 is $89.9 \%$, with a $14.4 \%$ standard deviation among the sensors. The highest data availability for a sensor is $98.2 \%$, while the lowest $27.4 \%$.

\section{Seasonal, hourly factors and AADT estimation}

Following the AADT estimation method described in Section 2.3.2, we estimate the corresponding traffic state for the parts of the segment associated with sensors where the data availability is low. For 38 of the 92 detector stations we managed to create complete AADT and AADS data, and hence these stations sites are considered as the PTC sites, while the remaining 54 detector stations, sites are assumed to be the SPTC. For the grouping of PTC sites, the Fuzzy C-means 
(a)

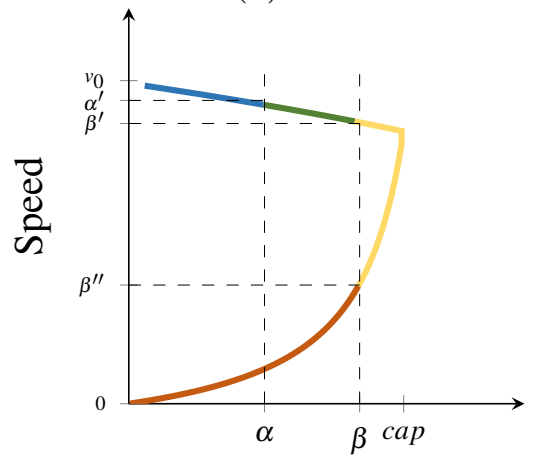

Flow (b)

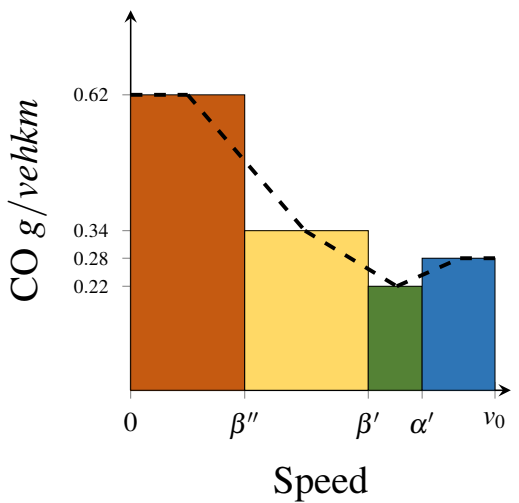

Heavy $\quad$ Free flow

Figure 3.2: (a) Linear hyperbolic fundamental diagram and derivation of speed thresholds (Speed $>\alpha^{\prime}$ : Free flow, $\alpha^{\prime} \geq$ Speed $>\beta^{\prime}$ : Heavy, $\beta^{\prime} \geq$ Speed $>\beta^{\prime \prime}$ : saturated, Speed $\leq \beta^{\prime \prime}$ : Stop and go); (b) CO emission factor as a discrete (bars) and as a continuous (dashed line) function of average speed.

algorithm (Bezdek et al., 1984) is used, similarly to Gastaldi et al., (2013). The Fuzzy C-means algorithms requires the optimal number of clusters as input. For the evaluation of the number of clusters we use the pseudo-F statistic criterion (Caliński and Harabasz, 1974). Finally, the optimal number of road groups is found to be $3,|I|=3$, for both speed and flow. The resulting time variation factors, $f_{i, j}$ are illustrated by Figure 3.3, while Figure 3.4 shows the hourly factors, $g_{d, i, h}$ for each road group $i$.

For each one of the SPTC sites, $\beta$, the annual average daily traffic, $q_{\beta, i}^{A A D T}$, is estimated through linear regression, and finally the SPTC site is assigned to a specific road group. The same methodology is used for the estimation of the annual average speed. Finally, the average hourly values of flow, $Q_{j, h}^{H T}$, and speed, $V_{j, h}^{H T}$, that are missing for each sensors, are estimated using the Equations (2.18) and (2.20).

\section{Spatial aggregation methods}

Cross-sectional data from stationary detectors needs to be extended in space, before a macroscopic emission model can be applied. A common approach for extending the measurements to network links, is to assume that traffic conditions are homogeneous for the whole 
(a)

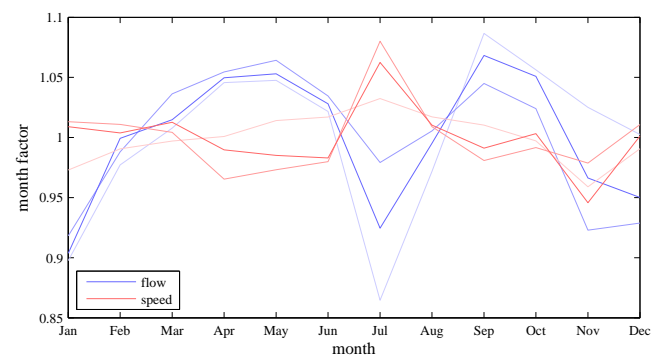

(b)

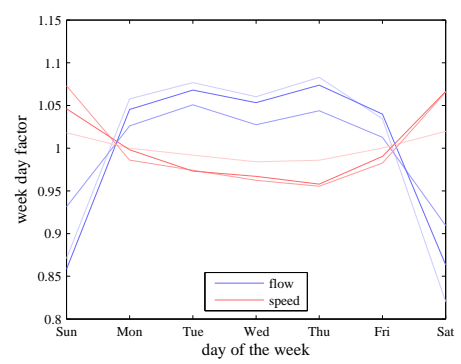

Figure 3.3: Seasonal factors (a) week day (b) month. The shades of blue and red are associated with different road groups.

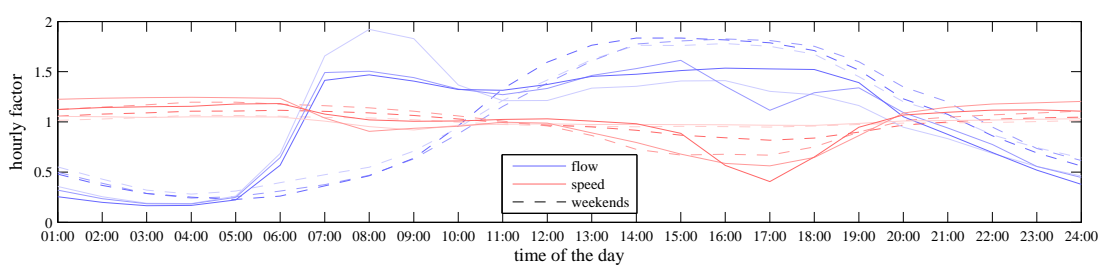

Figure 3.4: Flow and speed hourly factors, for the different road groups.

length of a link, referred to this study as the Average Homogeneous Segment (AHS) approach. Assume, that $S_{a}$ is the set of the sensors, $s$, located along the homogeneous link $a$. Let $L_{a}$ be the length of link $a$, and let $V_{j, h, s}^{H T}$ and $Q_{j, h, s}^{H T}$ be the hourly mean speed and flow, of each sensor $s$. Then, based on the spatially aggregated speed $V_{j, h, a}^{H T}$ and flow $V Q_{j, h, a}^{H T}$ the total emissions, $E_{j, h, a}^{p}$, for each link $a$ and pollutant $p$, during day $d$ and hour $h$, can be expressed as

$$
E_{j, h, a}^{p}=e^{p}\left(\frac{1}{\left|S_{a}\right|} \sum_{s \in S_{a}} V_{j, h, s}^{H T}\right) \cdot \frac{1}{\left|S_{a}\right|} \sum_{s \in S_{a}} Q_{j, h, s}^{H T} \cdot L_{a} .
$$

Alternatively, one can assume that each sensor has an Area Of Influence (AOI). This area could, for instance, be the half of the distance between two adjacent sensors. This can easily be achieved by divining the network into segments so that each segment contains exactly one sensor. Assume that the sensors $s$ are consecutively numbered based on their longitudinal position $x_{s}$. Then, the total emissions, $E_{j, h, s}^{p}$, of pollutant $p$, during day $d$ and hour $h$, at area of influence of sensor s, can be expressed as 


$$
E_{j, h, s}^{p}=e^{p}\left(V_{j, h, s}^{H T}\right) \cdot Q_{j, h, s}^{H T} \cdot \frac{\left(x_{s+1}-x_{s}\right)+\left(x_{s}-x_{s-1}\right)}{2} .
$$

\section{Emission estimation based on ASM}

ASM, described in Subsection 2.3.2, fully reconstructs the measured speed and flow field, filing the spatio-temporal gaps in the data. Therefore, ASM provides the appropriate traffic state and activity information required for an emission estimation analysis. Wang et al., (2011) in their study use ASM to estimate emissions from crosssectional data. However, they use a microscopic emission model and ASM is employed to produce vehicle trajectories. In this thesis, we utilize ASM to get the required traffic information for an macroscopic emission model. As mentioned in Subsection 2.3.2, $\kappa$ and $\tau$ are associated with the discretised space and time respectively. Given that $\lambda$ and $\mu$ are the lengths of each space and time interval, in kilometres and hours respectively, at each section $\kappa$ and time interval $\tau$, emissions can be computed as

$$
E_{\kappa, \tau}^{p}=e^{p}\left(V_{\kappa, \tau}\right) \cdot Q_{\kappa, \tau} \cdot \lambda \cdot \mu,
$$

where $Q_{\kappa, \tau}$ and $V_{\kappa, \tau}$, are the ASM based average flow in $v e h / h$ and speed in $\mathrm{km} / \mathrm{h}$.

Figure 3.5 illustrates the sensors outputs for a typical day of 2016. Note the cross-sectional nature of data and the lack of traffic situation information between sensors. Applying ASM, we obtain a continuous in space and time field of the traffic variables (Figure 3.6). Finally, based on the reconstructed speed and flow field, emissions for five pollutants are estimated using Equation 3.3. The space interval is set to 100 meters, therefore $\lambda=0.1$, while the temporal is set to 5 minutes, and thus $m=5 / 60$. The resulting emissions for $\mathrm{NO}_{\mathrm{x}}$ and $\mathrm{CO}$ are illustrated in Figure 3.7.

\subsection{Computational results}

This section presents the emissions resulting from three different approaches of obtaining the traffic data: (i) AADT combined with spatial aggregation AHS, (ii) AADT combined with or AOI and (iii) ASM, for two different cases. In the first case we take into account 
(a)

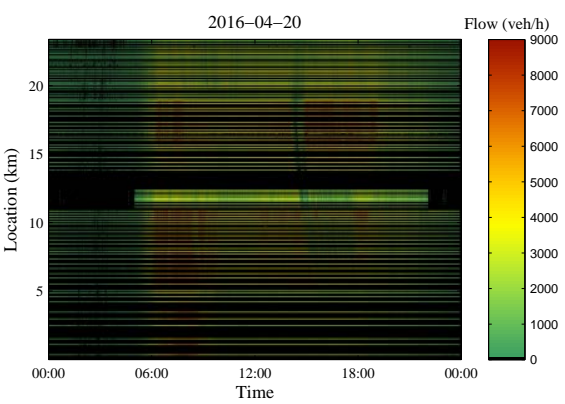

(b)

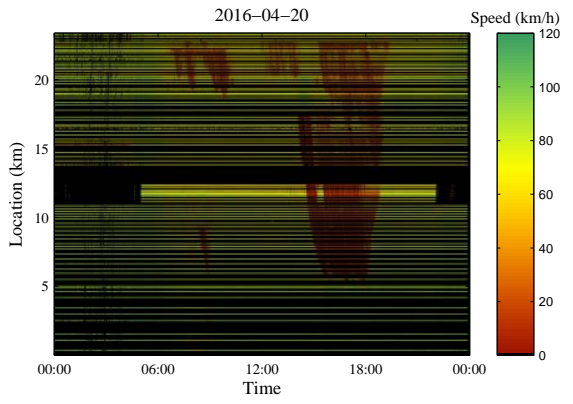

Figure 3.5: Cross-sectional data from stationary detectors (a) flow, (b) speed.

(a)

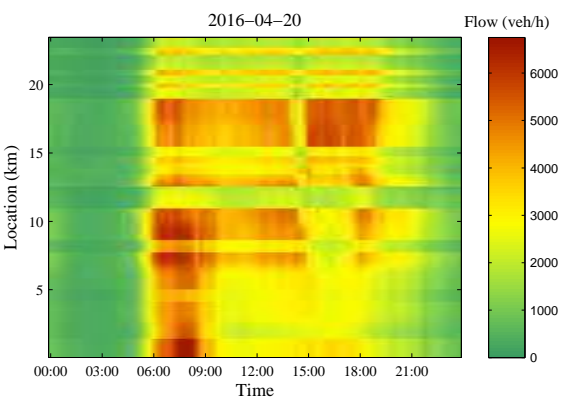

(b)

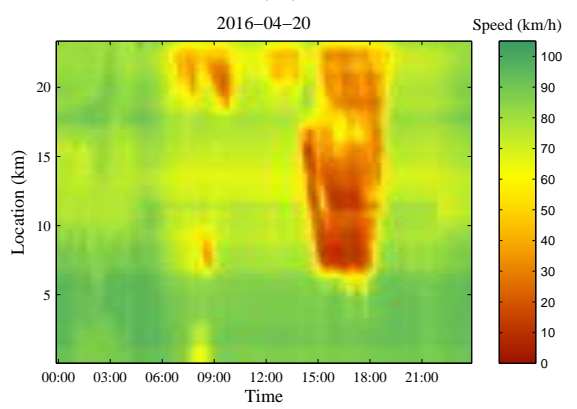

Figure 3.6: ASM based reconstructed field (a) flow, (b) speed.

(a)

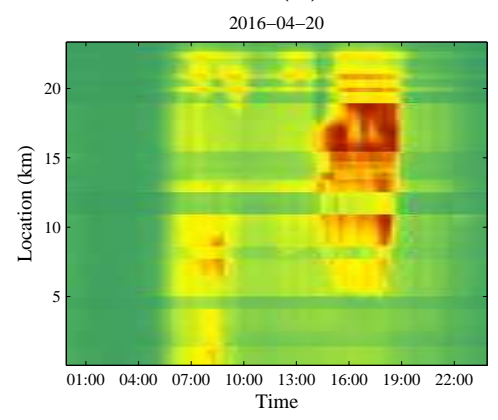

(b)

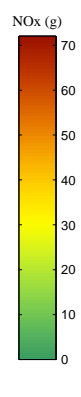

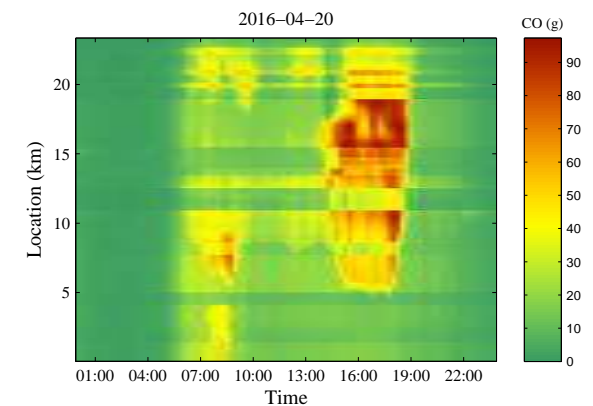

Figure 3.7: Emission estimation (grams per 100 metres and 15 minutes) (a) $\mathrm{NO}_{\mathrm{x}}$, (b) CO. 
Table 3.2: Annual emissions for case 1.

\begin{tabular}{llllll}
\hline \multirow{2}{*}{ Case 1: full data } & \multicolumn{5}{c}{ Tones of pollutant emitted } \\
\cline { 2 - 6 } & $\mathrm{HC}$ & $\mathrm{CO}$ & $\mathrm{NO}_{\mathrm{x}}$ & $\mathrm{CO}_{2}$ & $\mathrm{PM}$ \\
\cline { 2 - 6 } $\mathrm{ASM}$ & 10.500 & 120.07 & 103.60 & $6.14 \cdot 10^{4}$ & 1.6084 \\
$\mathrm{AADT}+\mathrm{AHS}$ & 10.390 & 118.80 & 102.29 & $6.06 \cdot 10^{4}$ & 1.5873 \\
$\mathrm{AADT}+\mathrm{AOI}$ & 10.554 & 120.05 & 103.35 & $6.12 \cdot 10^{4}$ & 1.6016 \\
\hline
\end{tabular}

Table 3.3: Percentage difference with the base-line, at a day level for the case 1 ( $\mathrm{Std}=$ Standard deviation).

\begin{tabular}{llllll}
\hline \multirow{2}{*}{ Case 1: full data } & \multicolumn{5}{c}{ Percentage difference with the base-line } \\
\cline { 2 - 6 } & Pollutant & mean & Std & $\min$ & $\max$ \\
\hline AADT +AHS & $\mathrm{HC}$ & -1 & 2.9 & -13.3 & 17.2 \\
& $\mathrm{CO}$ & -1.1 & 2.8 & -12 & 18.4 \\
\multirow{4}{*}{$\mathrm{AADT}+\mathrm{AOI}$} & $\mathrm{NO}_{\mathrm{x}}$ & -1.3 & 2.5 & -11.3 & 14.2 \\
& $\mathrm{HC}$ & 0.5 & 3.4 & -22.9 & 25.1 \\
& $\mathrm{CO}$ & -0.1 & 3.3 & -11.9 & 25.2 \\
& $\mathrm{NO}_{\mathrm{x}}$ & 0.2 & 2.7 & -10.9 & 18.8 \\
\hline
\end{tabular}

the full data set that is available for the 2016. In the second case, we assume that a significant part of the data is missing.

Case 1 (full data): The data availability for the SPTCs is for this case high $(84.5 \%)$, resulting in an accurate AADT estimation. Table 3.2 presents the annual total emissions for the case-study road segment, based on the three different approaches.

Assuming that ASM provides a reliable space-time field of speed and flow, the resulting emission estimates for the full dataset are regarded as the base-line emissions. In order to evaluate the accuracy of the other two approaches (AADT+AHS and AADT+AOI), their resulting emissions are compared against the base-line. In Table 3.2 we notice that at an annual level the differences between the approaches are relatively small, mainly due to the high data availability. However, considering specific days, the differences are high; Table 3.3 presents the mean, the standard deviation, the minimum and the maximum percentage difference among the 366 days of 2016, between the two approaches (AADT+AHS and AADT+AOI) and the base-line for the three pollutants $\left(\mathrm{HC}, \mathrm{CO}\right.$ and $\left.\mathrm{NO}_{\mathrm{x}}\right)$ where we noticed the highest differences. 
(a)

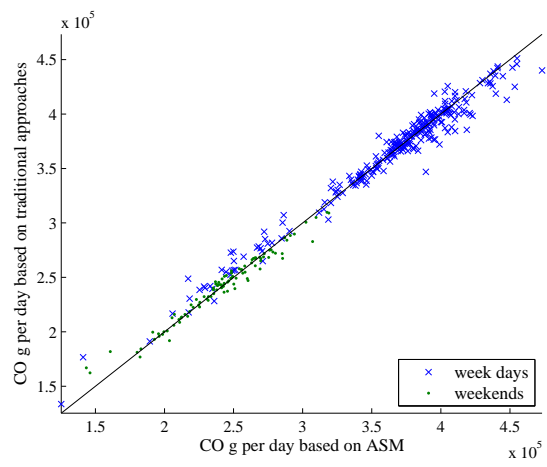

(b)

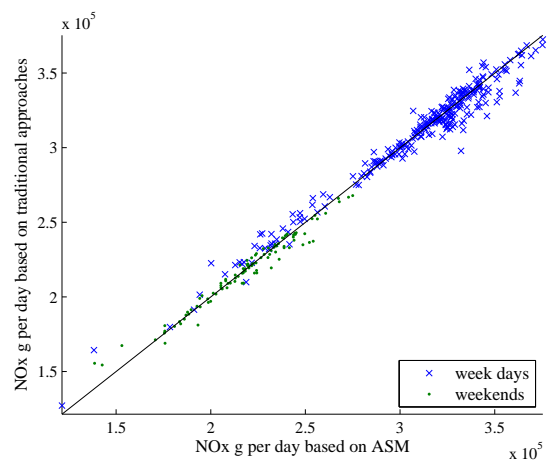

Figure 3.8: Emissions based on full data and the traditional approaches (AADT + AOI full data) versus the base-line (ASM full data) for each day of 2016 and for (a) $\mathrm{CO}$ (b) $\mathrm{NO}_{\mathrm{x}}$.

Although at an annual level the differences between the approaches are not so vital, at a daily level the overestimation could reach to $25.2 \%$ while the underestimation to $22.9 \%$. The differences can be even higher considering the spatial distribution of the estimated emissions. Figure 3.9 illustrates the resulting emissions for each 100 $m$ of the segment that are based on ASM versus the corresponding emissions that are based on the traditional approaches. The spatial allocation of annual emissions, per 100 metres, is illustrated by Figure 3.10. Despite the fact that the whole segment's annual estimated emissions do not significantly differ between the three approaches, according to Figure 3.10 their spatial allocation crucially changes. These differences can have a direct effect on the spatially sensitive applications of emission modelling.

Case 2 (reduced data): In large-scale networks the data availability usually is not so high as the one in our case-study segment. However, we chose this segment in order to construct a reliable base-line for our comparisons. In the case 2 the available data of the segment is significantly reduced in order to more realistic represent the data availability of a large network. By leaving only two weeks counts for 64 sensors, the average sensors data availability is diminished to $26.3 \%$ with a standard deviation of $39 \%$. The number of the PTC is reduced from 38 to 14 , while the data availability for the SPTC sites is declined to $13.5 \%$. For most of the SPTC, the assignment into the road groups and the AADT estimation is based on limited measurements with a two weeks duration. Table 3.4 presents the 
(a)

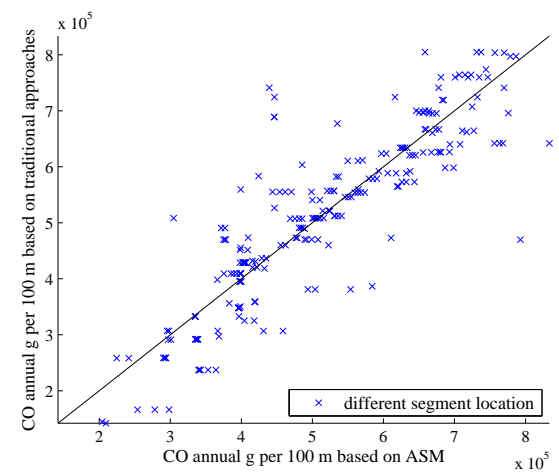

(b)

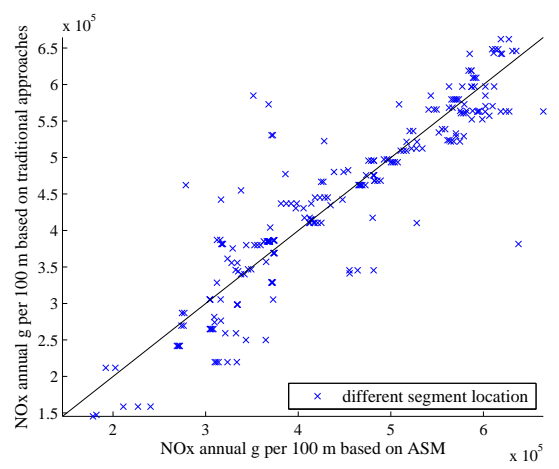

Figure 3.9: Annual emissions based on full data and the traditional approaches (AADT + AOI full data) versus the base-line (ASM full data) for each $100 \mathrm{~m}$ of the segment and for (a) CO (b) $\mathrm{NO}_{\mathrm{x}}$.

(a)

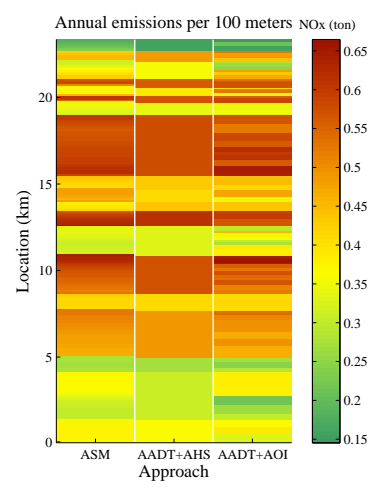

(b)

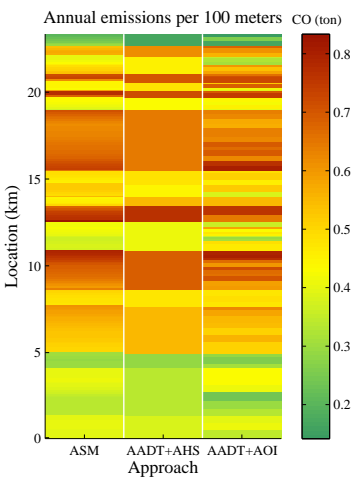

(c)

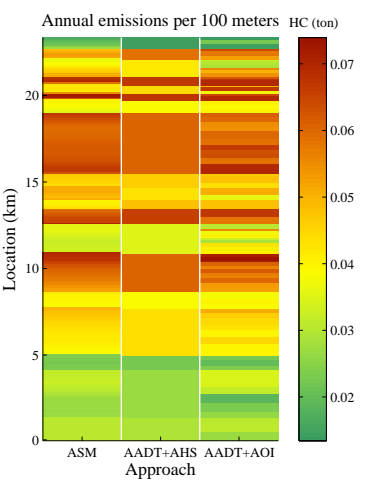

Figure 3.10: Annual emission estimates per 100 metres considering the case 1 (full data), and for the pollutants (a) $\mathrm{NO}_{\mathrm{x}}$, (b) $\mathrm{CO},(\mathrm{c}) \mathrm{HC}$. 
Table 3.4: Annual emissions for case 2 .

\begin{tabular}{llllll}
\hline \multirow{2}{*}{ Case 2: reduced data } & \multicolumn{5}{c}{ Tones of pollutant emitted } \\
\cline { 2 - 6 } & $\mathrm{HC}$ & $\mathrm{CO}$ & $\mathrm{NO}_{\mathrm{x}}$ & $\mathrm{CO}_{2}$ & $\mathrm{PM}$ \\
\cline { 2 - 6 } $\mathrm{ASM}$ & 10.42 & 118.59 & 102.41 & $6.09 \cdot 10^{4}$ & 1.587 \\
$\mathrm{AADT}+\mathrm{AHS}$ & 10.61 & 121.74 & 104.74 & $6.21 \cdot 10^{4}$ & 1.625 \\
$\mathrm{AADT}+\mathrm{AOI}$ & 10.30 & 123.88 & 106.33 & $6.30 \cdot 10^{4}$ & 1.647 \\
\hline
\end{tabular}

Table 3.5: Percentage difference with the base-line, at a day level for the case 2 (Std=Standard deviation).

\begin{tabular}{llllll}
\hline \multirow{2}{*}{ Case 2: reduced data } & \multicolumn{5}{c}{ Percentage difference with the base-line } \\
\cline { 2 - 6 } & Pollutant & mean & Std & $\min$ & $\max$ \\
\hline ASM & $\mathrm{HC}$ & -0.8 & 1.9 & -5.2 & 4.2 \\
& $\mathrm{CO}$ & -1.3 & 2 & -6.6 & 3.7 \\
AADT +AHS & $\mathrm{NO}_{\mathrm{x}}$ & -1.2 & 1.8 & -5.1 & 3.1 \\
& $\mathrm{HC}$ & 1.1 & 14.5 & -22.9 & 115.8 \\
& $\mathrm{CO}$ & 1.4 & 13.7 & -20 & 115.9 \\
AADT +AOI & $\mathrm{NO}_{\mathrm{x}}$ & 1.1 & 11.3 & -16.4 & 93.21 \\
& $\mathrm{HC}$ & 3.4 & 16 & -20.7 & 132.7 \\
& $\mathrm{CO}$ & 3.2 & 14.8 & -17.9 & 128.3 \\
& $\mathrm{NO}_{\mathrm{x}}$ & 2.6 & 11.9 & -14.8 & 101.4 \\
\hline
\end{tabular}

resulting annual estimated emissions for the three approaches related to this second case, while Table 3.5 presents the daily variation of the differences.

The level of annual differences between the three approaches and the base-line, for this case is again not very high. However, considering the results presented in Table 3.5, ASM seems to have a better performance when the data is reduced, especially at a daily level. Concerning the other two methods, both of them but especially the AADT + AOI approach, tend to overestimate the total annual emissions compared to the base-line. Nevertheless, the most significant difference between the two methods and the base-line relies on the temporal, at a day level, variations of the annual emissions.

Figure 3.11 illustrate the estimated emissions of $\mathrm{CO}$ and $\mathrm{NO}_{\mathrm{x}}$ based on AADT+AOI when the data is reduced versus the base-line. The results demonstrate that the estimated seasonal curves cannot efficiently capture the traffic variations and consequently the magni- 
(a)

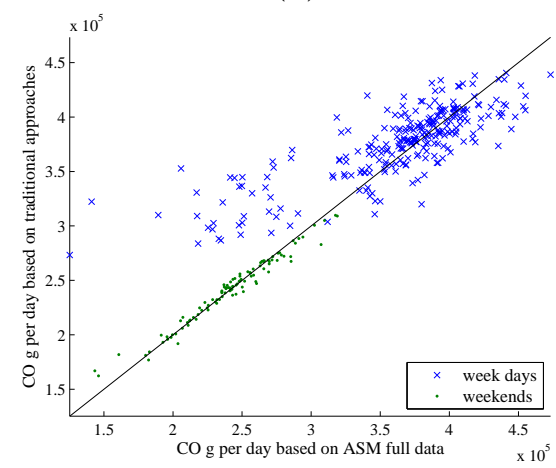

(b)

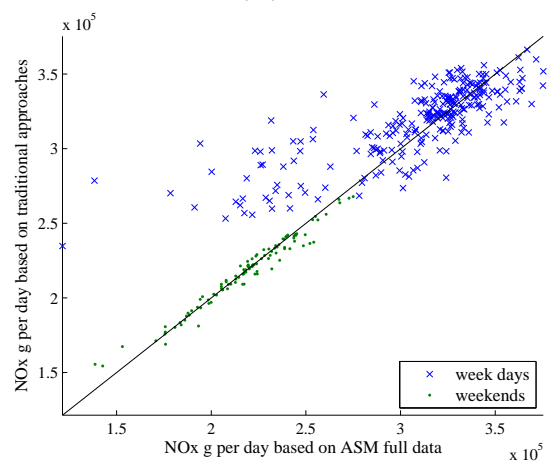

Figure 3.11: Emissions based on reduced data and the traditional approaches (AADT + AOI reduced data) versus the baseline (ASM full data) for each day of 2016 and for (a) CO (b) $\mathrm{NO}_{\mathrm{x}}$.

tude of the emitted pollutants for some extreme days (either intensely congested or days with sparse traffic). On the contrary, ASM, being capable to estimate the propagation of traffic perturbation, performs better with limited input data (Figure 3.12).

It is important to note here that although ASM had a better performance, computational time and required computer memory, remain an issue, since they are significantly higher compared to the other two approaches. The computational time of ASM, can be influenced by the input data size, but mainly depends on the length of the space and time discretisation intervals $\lambda, \mu$. For that reason, we experiment with different sets of spatio-temporal aggregation values. The accuracy and the efficiency of the aggregation is evaluated based on the emission estimation results. The results presented in Table 3.2 and Table 3.4 are based on our most detailed estimation, where the discretisation space interval is 100 metres and the time interval is 15 minutes (ASM 100/15). By gradually increasing the aggregation level, we examined the effect on computational time, data size and annual emissions estimates. The first column in Table 3.6 describes the considered alternatives regarding the spatial (metres)/temporal (minutes) aggregation levels. The rest columns contain the values of the percentage differences between each alternative and the ASM $(100 / 15)$.

We can conclude that by modifying the aggregation level, the magnitude of the estimated emissions is not drastically changed, while 
(a)

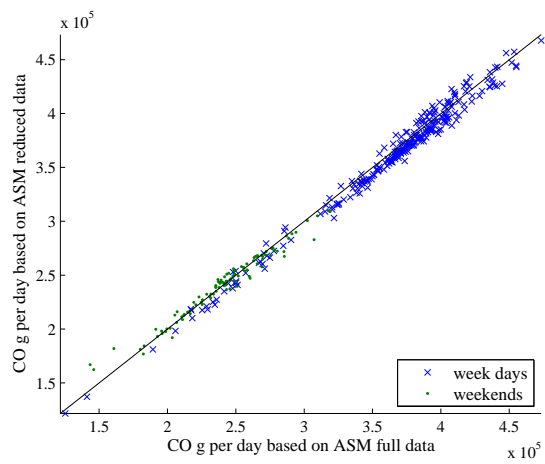

(b)

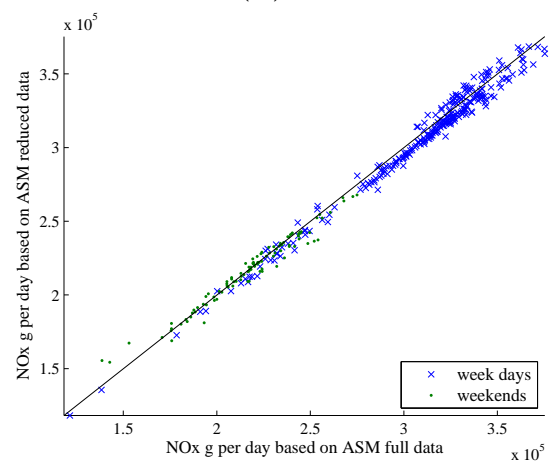

Figure 3.12: Emissions based on ASM (reduced data) versus the base-line (ASM full data) for each day of 2016 and for (a) $\mathrm{CO}(\mathrm{b}) \mathrm{NO}_{\mathrm{x}}$.

Table 3.6: The effects of ASM aggregation level (Com. time $=$ Computing time, Req. memory=Required memory).

\begin{tabular}{|c|c|c|c|c|c|}
\hline \multirow{2}{*}{ Case 2} & \multicolumn{5}{|c|}{ Percentage difference with ASM 100/15 } \\
\hline & $130 / 25$ & $150 / 30$ & $160 / 40$ & $180 / 50$ & $200 / 90$ \\
\hline $\mathrm{HC}$ & 0.2903 & 0.1240 & 0.0111 & 0.4362 & 1.0132 \\
\hline $\mathrm{CO}$ & 0.4382 & 0.3541 & 0.2831 & 0.7062 & 1.4913 \\
\hline $\mathrm{NO}_{\mathrm{x}}$ & 0.3078 & 0.2746 & 0.3738 & 0.6888 & 1.8053 \\
\hline $\mathrm{CO}_{2}$ & 0.2911 & 0.2784 & 0.4091 & 0.7070 & 1.9365 \\
\hline PM & 0.3061 & 0.3031 & 0.4293 & 0.6957 & 1.9266 \\
\hline Com. time & -65.18 & -75.89 & -83.93 & -88.39 & -96.73 \\
\hline Req. memory & -52.76 & -65.17 & -74.88 & -81.69 & -85.44 \\
\hline
\end{tabular}


the computing time and the required memory are significantly decreased. If we compare the estimated emissions from ASM 160/40 to the base-line (ASM 100/15 full data), the total annual emissions are underestimated from $0.65 \%$ to $1.63 \%$ depending on the pollutant, while the standard deviation of this underestimation between the 366 days of the year ranges from $1.7 \%$ to $2.2 \%$. Therefore, ASM with longer space and time discretisation intervals can more efficiently fill the spatio-temporal gaps. We should note here that longer space and time intervals may be more appropriate to use also from an HBEFA modelling perspective. HBEFA is a macroscopic emission model and HBEFA emission factors represent average traffic conditions.

\subsection{Discussion}

The installation of stationary detectors is a cost-efficient method of collecting the traffic data required during an emission estimation analysis. However, a temporally comprehensive data set is not always feasible for every road of a network and cross-sectional measurements is by their nature non-continuous in space.

Traditionally, the temporal variations of traffic are estimated by the application of seasonal factors over an estimated AADT, while spatially homogeneous segments are considered. Since the spatiotemporal variations of traffic conditions is a crucial factor from an emission estimation point of view, in this study we suggest the use of a more detailed traffic state estimator (ASM). Having cross-sectional data as input, ASM provides a complete space and time field of the traffic variables. Considering two different cases of data availability, we estimate the traffic state for a whole year, first based on ASM and then based on the traditional traffic estimation approaches. Next we compare the resulting from ASM emission rates with the corresponding ones derived from the traditional traffic estimating approaches.

For the first case, where the temporal availability of cross-sectional data is high, the main differences between the approaches are associated with the temporal and spatial distribution of the total annual emissions. However, despite the fact that the spatial allocation of emissions differs significantly, we noticed convergence among the different approaches regarding the total annual emissions. Concerning the case of reduced temporal data availability, the differences between the variations in terms of daily traffic, are higher, mainly due to the 
fact that AADT and seasonal factors cannot describe extreme days. ASM showed a better performance, being able to describe the traffic variations more accurately.

Therefore, considering the research Q1 of this thesis, we can conclude that for applications of emissions such as NEI, the use of a more detailed traffic state estimator may not be necessary, since the traditional approaches provide sufficiently accurate results. However, for applications such as air quality modelling, which are sensitive to spatio-temporal variations of emissions, ASM leads to more reliable results, especially when the availability of cross-sectional data is limited.

Computational time and memory requirements are an issue for ASM, which limits the usage mainly to off-line estimation. However, by increasing the length of the spatial and temporal discretisation intervals, the computing effort can be reduced without significant affecting the quality of the results.

Additionally, we should highlight here that our results can be regarded as case specific, since the geographical and other characteristics of each segment are similar. We had a relatively accurate AADT estimation (compared to many real world cases), leading to not so high differences regarding the annual emissions. Furthermore, we used linear regression for the AADT estimation. However, Zhong et al., (2004) conclude that neural networks could have better performance. Furthermore, it would also be interesting for the future to use emission related seasonal factors, instead of the traditional flow seasonal factor estimation.

Moreover, we consider constant vehicle fleet composition, and the emission factors are only depending on average speed and road type. It would be of interest in future to exploit the sensor data for a dynamic and location specific estimation of vehicle fleet composition. 


\section{Chapter 4}

\section{Emission estimation based on static traffic models}

Since it is not feasible to install and maintain traffic flow sensors throughout a whole network, traffic models are an alternative approach for generating the required traffic input. Also, traffic models are important tools for planning purposes, and commonly joint with emission analyses. The thesis focuses on the last step of transportation planning models, traffic network assignment, examining how emission modelling is affected by different assignment approaches. Although DTA approaches can be considered as more suitable for providing traffic data to emission models, due to complexity and calibration issues most emission estimations are based on outputs of STA models.

Section 4.1 describes how the outputs of a STA model can be incorporated in an emission modelling analysis. Additionally, in Section 4.1 the problems associated with the use of STA for generating the input traffic data to emission models are described. In the same section we also categorise these issues as problems either related to the large aggregation periods or problems regarding the static network loading. The main goal of this chapter is to examine if the inherent simplistic nature of STA can influence the final emission estimates and lead to under- or over-estimations. In Section 4.2 we attempt to 
identify and quantify those errors using the same case-study area as in Chapter 3. Finally, Section 4.3 discusses the main findings of this chapter and aims to give answer to research Q2 of the thesis.

\subsection{Definition and categorisation of the problems}

HBEFA emissions factors are originally given as a discrete function of traffic situation. When the traffic situation has to be determined from a STA model, this distinction is usually based on V/C thresholds, such as the thresholds suggested by the Swedish Traffic Agency Handbook for road traffic and air pollution (Trafikverket, 2012) presented in Table 3.1.

In this way, the optimal link flow obtained from the solution of the STA (Problem 2.12), is used to determine the traffic situation for each link in the network. Then, with the traffic situation known, the emission factor, $\tilde{e}^{p}$, for every link $a$ and pollutant $p$ can be determined. Next, the emission factor multiplied by the traffic activity expressed in vehicle kilometre travelled, $x_{a} \cdot L_{a}$, where $L_{a}$ is the length of link $a$, gives the total grams of each pollutant, $p$, emitted at link $a, E_{a}^{p}$,

$$
E_{a}^{p}=\tilde{e}_{a}^{p} \cdot x_{a} \cdot L_{a} .
$$

STA problem has unique solution if VDF satisfies the conditions (2.13), namely if the travel cost of each link does not depend on the traffic volume of other links. In this way, links interactions are implicitly not considered and due to static network loading, important traffic flow phenomena, such as spill-back, are neglected. More specifically, in the case of a bottleneck, $(V / C>1)$, all the time delays and excess emissions arise at the bottleneck link, while the links upstream the bottleneck remain unaffected. However, in reality higher delays and emissions are observed upstream the bottleneck, due to the propagation of the queues. Nevertheless, a well calibrated STA model may result to an accurate total route travel time, since the modelled delays at the bottleneck could compensate the delays, observed in reality upstream the bottleneck. Although the accurate spatial allocation of delays may not be so important in the case of travel time, in the case of emissions that have location specific effects, interactions of links become a significant factor. 
When a traffic analysis is oriented towards travel times, the most critical aspect is the total route travel time, namely the time that travellers need to reach from their origin to their destination. The exact location of the congestion, where the travellers actually have experienced the delay, is not usually of great importance. However, the accurate estimation of congestion's location becomes significant from an air quality, dispersion and exposure modelling point of view. Using location specific meteorological, demographic and background pollution data but with spatially inaccurate distribution of road emissions, can lead to an erroneous exposure. The latter could have a direct effect during an economic analysis, e.g. cost-benefit analysis, where the monetary cost of grams of pollutant emitted is analogous to the number of people exposed and affected (Eliasson, 2009). Accordingly, in such an analysis, the travel time costs are associated with route travel times. Thus, STA may be sufficiently accurate to predict path travel times, since they don't depend on the delay location. In most of the cases, though, STA is the basis for both travel times and emissions estimation. However, it should be highlighted here that this is not the case for all the types of pollutants, for instance $\mathrm{CO}_{2}$ has long-term and global effects and therefore the total network's emissions are only of interest.

The first group of problems that potentially can lead to emission estimation errors is therefore related to static loading and the incorrect location of congestion. Another modelling assumption of STA models that can also influence the emission estimation, is related to the aggregation of traffic variables in time and space. The traffic conditions are assumed to be temporally constant during the whole analysis period, while links are assumed to be spatially homogeneous. Thus, traffic variables are expressed in both temporal and spatial averages. Therefore, STA cannot capture the variations of traffic conditions, which are crucial from an emission modelling perspective. The difficulty of using mean speeds and flows as input to emission models have been discussed in several papers (Nesamani et al., 2007; Muller-Perriand, 2014; Ryu et al., 2015).

\subsection{Quantification of the errors}

The two basic limitations associated with STA described in the previous section can introduce errors into the emission modelling. In 
this section we attempt to quantify these errors. For the same case study road segment as in Section 3.3, we compute emissions based on STA model outputs. Next, we compare the resulting emissions with emissions which rely on the ASM approach and we evaluate the results.

\subsubsection{Methodology}

In this section we use as a case study part (19-km long) of the segment described in Section 3.3 (see Figure 4.1), where the most congestion is observed. We divide the section in 27 homogeneous links based on the location of the on-ramps and off-ramps. During the morning peak one of the links becomes an active bottleneck resulting in queues spreading over several of the upstream links. For this reason, we set our analysis period to be the morning peak between 6:30 and 9:30, which is also a sufficiently long-time interval for the complete formation and dissolution of the queues. Additionally, the road section is equipped with radar sensors, measuring average speed and flow per lane during one-minute intervals. The sensors data provides a sufficiently realistic description of the traffic conditions and thus we use this data to construct the ground-truth for our experiments. Specifically, counts from three months (January, February and March in 2013) are considered. Based on the traffic state and traffic activity obtained by the radar sensors counts of speed and flow, we, initially, estimate emissions for the motorway section. These estimates are considered as ground-truth. Next, from the same stretch we estimate emissions based on $\mathrm{V} / \mathrm{C}$ ratios and a static model, considering a stable demand during the analysis period. By comparing these emissions with the ground-truth, we can calculate the errors that a static traffic model can introduce in the emission modelling.

To construct a more reliable ground-truth, capable of describing the spatio-temporal variations of traffic conditions, together with the sensors data we use the ASM methodology described in Section 3.3. Figure 4.2 depicts the reconstructed space-time speed field for the considered motorway section and for a typical day. Considering this figure, it will be helpful here to give a small description of the network's traffic situation during the morning peak. The upstream end of the bottleneck link is located at the position of $10.8 \mathrm{~km}$, that is also the starting point of the downstream propagated queues. The four lanes upstream this point, become two downstream, decreasing 


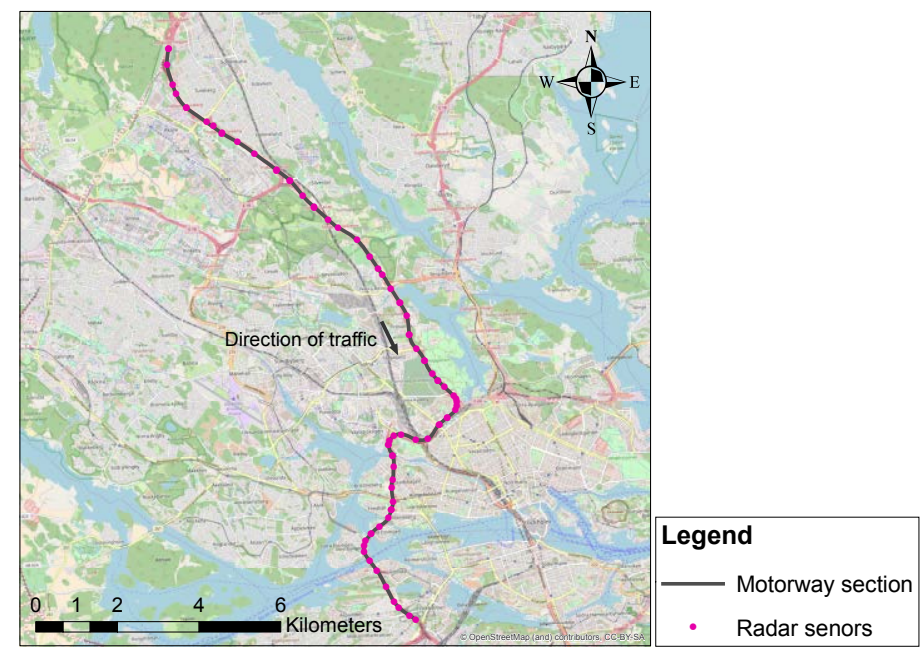

Figure 4.1: Modelled motorway section and the sensors position.

the capacity and creating a lane-drop bottleneck. The bottleneck is mainly active during the second hour of our analysis period. The position of $12.1 \mathrm{~km}$ is associated with the downstream end of the bottleneck link.

Following the methodology described in Subection 3.3 and using the Equation (4.2) we compute the grams of pollutant $p, E_{\kappa, \tau}^{p}$, emitted for each discretisation interval $\kappa, \tau$ and for the analysis period of each day.

$$
E_{\kappa, \tau}^{p}=\tilde{e}_{\kappa}^{p}\left(V_{\kappa, \tau}\right) \cdot Q_{\kappa, \tau} \cdot \lambda \cdot \mu .
$$

Figure 4.3 depicts the $\mathrm{NO}_{\mathrm{x}}$ emission field for a typical day. We should note here that we use the original discrete version of HBEFA, where emission factors are expressed as discrete functions of average speed. Among the 35 days, the average estimated emissions field is used as ground-truth for our experiments.

\subsubsection{Errors due to spatio-temporal aggregation}

As we have mentioned in the introduction of this section, we categorise the errors that static modelling can introduce to the emission estimation in two types: errors that concern the spatio-temporal resolution of average speed and flow, and errors related to inaccurate 


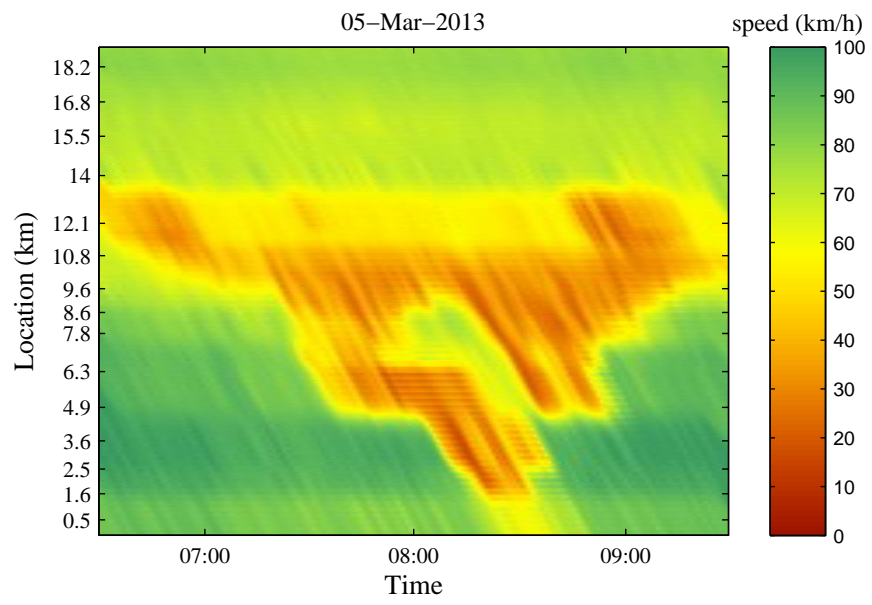

Figure 4.2: Speed field as a function of space and time, for the morning peak hours of a typical day.

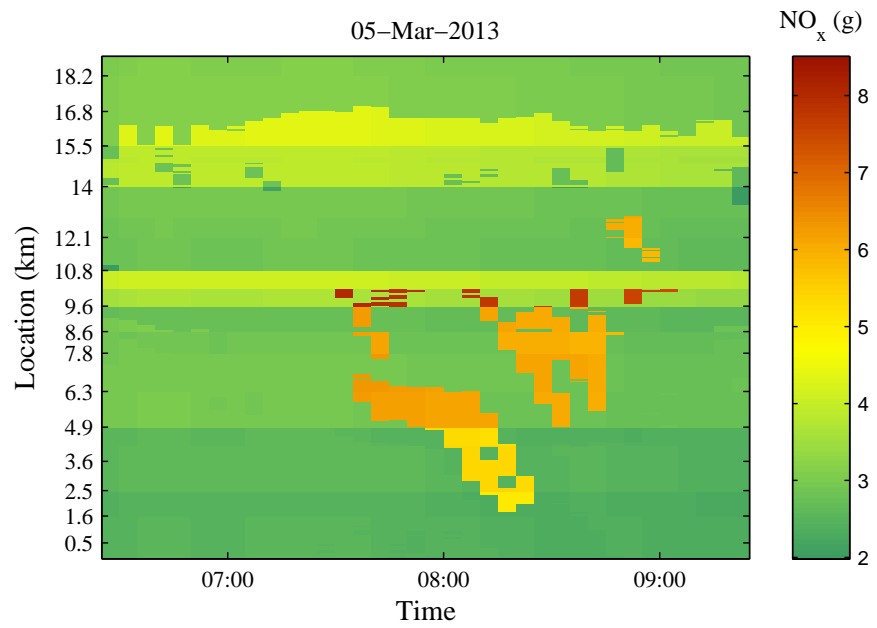

Figure 4.3: $\mathrm{NO}_{x}$ space-time field with $\lambda=50$ meters and $\mu=5$ minutes, for the morning peak hours of a typical day of our analysis period. 
congestion's location. If we assume that STA can accurately predict the traffic conditions, similarly to the ASM, but aggregated over longer spatial and temporal intervals, we can isolate the errors that concern the first category. We calculate emissions using the traffic estimator outputs aggregated spatially at a link level and temporally at an 3 hourly level as

$$
\begin{aligned}
& \tilde{E}_{\kappa, \tau}^{p}= \\
& \frac{1}{\left|\mathcal{D}_{a}\right|} \frac{1}{|\mathcal{T}|} \sum_{\kappa \in \mathcal{D}^{\kappa}} \sum_{\tau \in \mathcal{T}} Q_{\kappa, \tau} \cdot \lambda \cdot \mu \cdot \tilde{e}^{p}\left(\frac{1}{\left|\mathcal{D}^{a}\right|} \frac{1}{|\mathcal{T}|} \sum_{\kappa \in \mathcal{D}_{\kappa}} \sum_{\tau \in \mathcal{T}} V_{\kappa, \tau}\right), \\
& \forall \tau \in \mathcal{T}, \forall \kappa \in \mathcal{D}^{a}, \forall a \in \mathcal{A},
\end{aligned}
$$

where $\mathcal{T}=\left\{1,2, \ldots, n_{\tau}\right\}$ and $n_{\tau}$ is the number of the time intervals contained in 3 hours analysis period, $n_{\tau}=3 \cdot 60 / \mu, \mathcal{D}^{a}$ is the set of the space intervals, $\kappa$, included in link $a$, and $\mathcal{A}$ the set of the segment's links.

If we assume that a STA model is accurate and the errors are only related to the spatio-temporal aggregation, the emissions $\tilde{E}_{\kappa, \tau}^{p}$ correspond to the emissions derived from this "perfect" STA model. To quantify the influence of using longer aggregation intervals we compare the emissions $\tilde{E}_{\kappa, \tau}^{p}$ with the ground-truth, $E_{\kappa, \tau}^{p}$. For a more illustrative way to present our result we introduce here the term Errors Due to Spatial and Temporal Aggregation (ESTA), given by

$$
E S T A_{\kappa, \tau}^{p}=\frac{\tilde{E}_{\kappa, \tau}^{p}-E_{\kappa, \tau}^{p}}{E_{\kappa, \tau}^{p}} 100 .
$$

ESTA represents the emission under- or over-estimations in percentage, resulting from the spatio-temporal aggregation, for pollutant $p$ and for each space and time interval, $\kappa, \tau$.

Being consistent with our initial hypothesis, Figure 4.4 (a), demonstrates that longer aggregation periods significantly affect the emission estimation. The underestimation at specific locations can reach up to $40 \%$ for sensitive to speed variations pollutants such as the $\mathrm{HC}$ and the $\mathrm{CO}$. The underestimations are mainly located where the congestion is observed, namely upstream the bottleneck. At the congested links the speed variations are higher and thus, there is a clear correlation between ESTA and congestion. Moreover, the error depends on the link length, since in long links the spatial average speed 
(a)

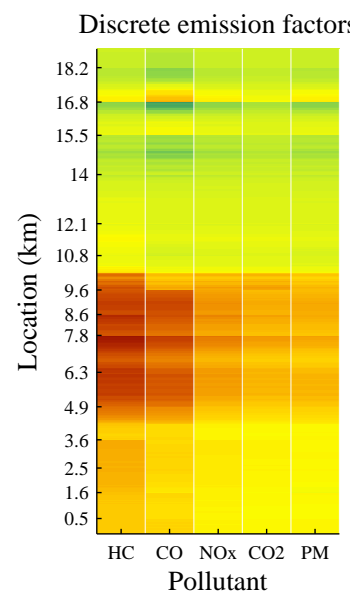

(b)

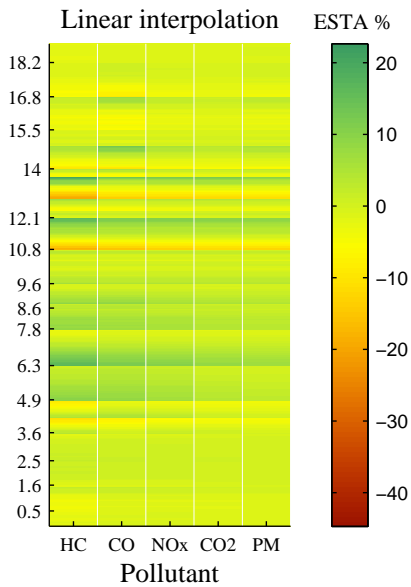

Figure 4.4: Average errors over the analysis period emission due to spatial and temporal aggregation (ESTA) when HBEFA emission factors are expressed as a (a) discrete (b) continuous function of average speed.

cannot capture the spatial variations. Therefore, even if we assume that a static model would accurately predict the same speeds and flows with the measurements, there are still substantial errors because of the low spatio-temporal resolution.

However, by applying the continuous version of HBEFA, as presented in Section 3.3, using $e^{p}(v)$ instead of $\tilde{e}^{p}(v)$ in Equation (4.2), the errors caused by the spatio-temporal resolution can be significantly reduced. According to Figure 4.4 (b) the emissions calculations become less sensitive to the aggregation period when the continuous version is used. Figure 4.5 illustrates the emission space-time field, when the HBEFA emission factors are given as continuous function of speed. The upstream end of the bottleneck, located at the position of $10.8 \mathrm{~km}$, is more distinct in this figure.

\subsubsection{Errors due to static loading}

In this section we attempt to identify and quantify emission errors that concern the inaccurate modelling of congestion's location by static models. For this reason, we compare our ground-truth with emissions estimation derived from static modelling outputs. Considering the fact that there is no queue formed before the start of the 


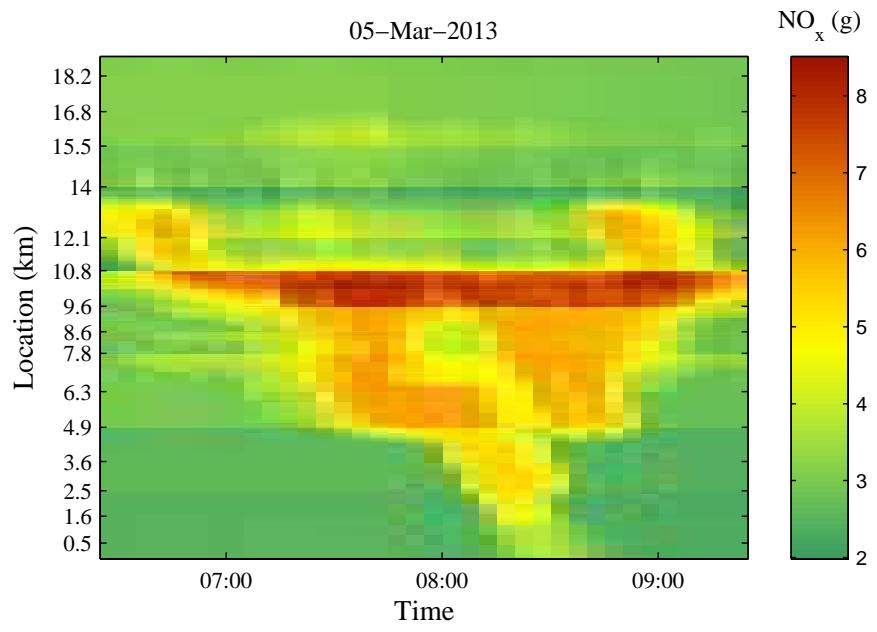

Figure 4.5: $\mathrm{NO}_{x}$ space-time field using the continuous version of HBEFA, for the morning peak hours of a typical day.

first hour, and all the queues have been discharged after the end of the third hour of the analysis period, we assume that the three-hour demand is equal to the total three-hours inflow of the segment. The bottleneck is active only during the second, intermediate hour of the considered analysis period. During the first and the third hour traffic conditions are stable, and since there are not any dynamic traffic flow phenomena observed, we assume that a well calibrated static model can lead to sufficiently accurate emission estimations. Therefore, the first and the third hour are excluded from our experiments. The demand period is divided, then, into three smaller, one hour long subperiods based on the demand variation indices suggested in Björketun and Carlsson, (2005). Specifically, to the first hour (6.30-7.30), $27 \%$ of the total demand is assigned, while to the second (7.30-8.30) and to the third (8.30-9.30), 37.5\% and $35.5 \%$ respectively. The capacities as well as the other parameters of the static model are estimated from calibration, using the flow and the speed counts from the radar sensors.

For a comprehensible illustration of the results we introduce the term Excess due to Congestion Emissions $E C E_{\kappa, \tau}^{p}$, defined as

$$
E C E_{\kappa, \tau}^{p}=Q_{\kappa, \tau} \cdot \lambda \cdot \mu\left(e^{p}\left(V_{\kappa, \tau}\right)-e^{p}\left(V_{\kappa}^{0}\right)\right),
$$


while the percentage difference given by

$$
E C E P_{\kappa, \tau}^{p}=\frac{e^{p}\left(V_{\kappa, \tau}\right)-e^{p}\left(V_{\kappa}^{0}\right)}{e^{p}\left(V_{\kappa}^{0}\right)} 100,
$$

where $V_{\kappa}^{0}$ is the free flow speed of space interval $\kappa$, and $e_{\kappa, i n}^{p}\left(V_{\kappa}^{0}\right)$ the emission factor, if at time $\tau$ and space $\kappa$ there was not any congestion. Therefore, ECEP expresses the difference in emission estimates between the current situation and the hypothetical situation where there would be not congestion at all. In this way we can isolate the additional emissions resulting from congestion and obtain their exact location. We should note here that the continuous version of HBEFA is used.

Figure 4.6 illustrates the value of ECEP at every location of the segment for both ground-truth and STA. In Figure 4.6 (a) we can notice that the excess emissions due to congestion, are located between the positions 10.8 and 9.6, namely at the first links upstream the bottleneck. Additionally, there are also some excess emissions upstream the location of $9.6 \mathrm{~km}$ because of the queues' spill-back. Especially, for some pollutants which are sensitive to congestion, such as the $\mathrm{HC}$, excess emissions are high even for the first kilometres of the segment.

In contrast to the ground-truth, the static model, without considering any capacity constraint assigns all the delays at the bottleneck link, due the high link's $V / C$ ratio (Equation (2.15)). Consequently, the excess emissions are also gathered at the same location. Figure 4.6 (b) demonstrates this fact since the highest excess emissions are located between the positions of $10.8 \mathrm{~km}$ and $12.1 \mathrm{~km}$, namely at the bottleneck link. The static model is not able to capture the propagation of the queues, neglecting an important part of the network's emissions. Moreover, according to the static model, higher excess emissions than the ground-truth are estimated downstream the bottleneck, especially for the $\mathrm{CO}$. The demand ratio may be high compared to the capacity for some links downstream the bottleneck, but in reality, the inflow rate at those links is equal to the bottleneck's capacity. The static model, which does not take any spill-back into account, assigns the whole demand, overestimating the emissions downstream the bottleneck. However, the differences regarding the total segment's emissions are low ranging from $1.30 \%$ to $1.42 \%$ depending the pollutant, because the excess emissions located at the bottleneck compensate for the excess emissions downstream the bottleneck observed in reality. Although the differences in the total seg- 
(a)

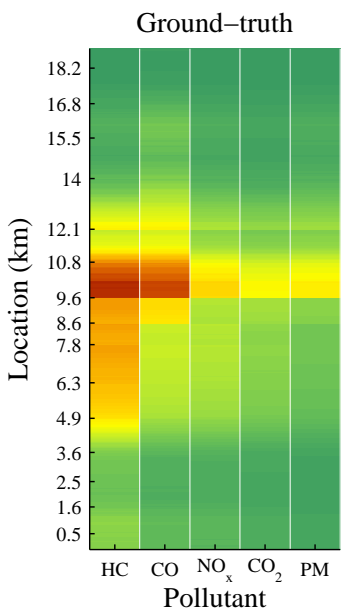

(b)

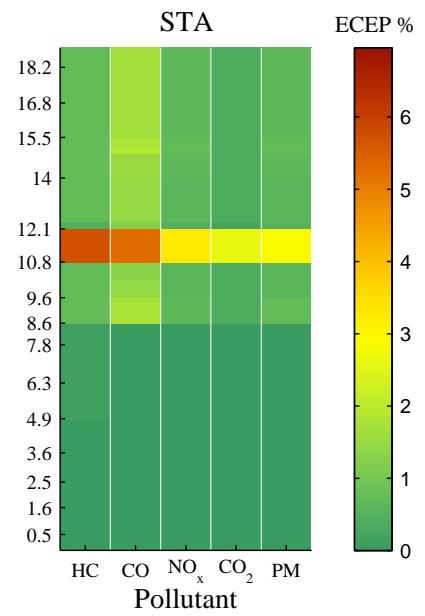

Figure 4.6: Excess due to congestion emissions based on (a) the ground-truth (b) static loading.

ment's emissions, between the ground-truth and STA are low, the excess emissions location differs significantly, leading to the undesired effects discussed in Section 4.1. Therefore, from an emission modelling perspective, an ideal model should be able to propagate the queues and as well as to block the paths after the bottleneck up to bottleneck's capacity, predicting the congestion's location accurately.

\subsection{Discussion}

For large scale emission estimation analysis, the required traffic conditions are usually obtained by static models. In this chapter, we show that the simplified way of representing traffic can lead to considerable emission estimation errors, giving an answer to research Q2 of this thesis. Temporal and spatial average traffic conditions tend to underestimate the emissions, especially in the case of a discrete emission models such as the HBEFA. Nevertheless, we demonstrate that using a continuous emission model, the spatio-temporal resolution problem can be satisfactorily moderated.

In addition, the STA models' inability of describing dynamic traffic flow phenomena can also cause significant emissions estimation errors. The inaccurate modelling of congestion's location may lead 
to unreliable spatial distribution of emissions. More specifically, the excess emissions can be estimated at the bottleneck link, instead of upstream the bottleneck where they are observed in reality. Even though STA results can be sufficient for travel time based analyses, we support that they need supplementary post-processing in the case of emission estimation. 


\section{Chapter 5}

\section{Improved emission estimation through post-processing}

In Chapter 4, we show that static models can lead to significant emission estimation errors, related to static network loading and to poor spatio-temporal resolution of traffic variables. These errors can have a direct effect on air quality and exposure modelling. Conversely, dynamic traffic models include representation of the traffic dynamics and might therefore be better suited as a basis for computing reliable emissions. However, they require additional computational time and calibration. Furthermore, such models cannot guarantee a unique equilibrium solution and therefore are often unsuitable for policy analyses. An alternative approach, which requires lower computing power than DTA but at the same time provides a more detailed representation of traffic conditions than STA, is the post-processing of STA outputs.

Section 5.1 gives an overview of the post-processing methods aiming to an improved emission estimation, applied until now. Our hypothesis is that a post-processing technique capable to capture link interactions can lead to more accurate emission estimation. Such a post-processing approach is presented in Section 5.2 and its use is tested and evaluated in Section 5.3.1 considering the same case-study road segment as in Chapter 4. In Section 5.3.2 the network effects 
of the suggested post-processor are examined. Section 5.4 concludes and discusses the main results. Chapter 5 aims to give an answer to research Q3 of this thesis.

\subsection{Post-processing of static modelling re- sults}

During the past years, several studies have highlighted the problem of using average speeds from static models in emission estimation. Some of these studied have attempted to address this issue by proposing post-processing techniques. Many researchers have moved towards the development of congestion correction techniques, capable to reflect the speed spatio-temporal variability (Negrenti, 1999; Nesamani et al., 2007; Smit et al., 2008; Ryu et al., 2015). The approach suggested in Negrenti, (1999) is based on a congestion correction factor, representing the speed variability along a link, and which can finally improve the accuracy of emissions estimations. Additionally, the proposed model in Nesamani et al., (2007) can provide a more precise prediction of emissions, by capturing traffic variations. Focusing on a set of factors that correlate congestion on each link, a dynamic variation in speed is constructed. Smit et al., (2008) also highlight the significant underestimations of emissions when using assignment outputs such as the average link speed. They identify that speed may vary on a link, supporting that the emission estimation can be improved by using speed distributions instead of a mean link speed. Finally, in Ryu et al., (2015) a corrected average speed model is suggested, by analysing the traffic characteristics that can cause emission estimation errors.

Bai et al., (2007b) consider direct speed post-processing techniques for improving emission estimates, based on intersection delays and queuing analysis approaches. Their methods mainly rely on the speed-flow curve and queueing analysis proposed by Akcelik, (1991a) and Dowling and Skabardonis, (1992). They conclude that that different post-processors generate dissimilar impacts on emissions. However, by applying such post-processing methods, queues are represented as vertical points and propagating phenomena like spill-back are again not taken into account. As is mentioned in Dowling and Skabardonis, (1992), by applying such post-processing techniques it is not feasible to track the upstream or downstream impact 
of queuing.

All the aforementioned approaches mainly focus on post-processing of link speeds, without taking the network topology and interactions between links into consideration. An alternative approach would be to apply a simulation-based network loading. Such techniques are commonly applied in dynamic traffic assignment models, and can be distinguished in micro, meso or macroscopic simulation approaches. All such approaches rely on dynamic route and demand information, and the quality of their results is largely depending on the calibration of the model. Micro and meso approaches require more detailed input about the road infrastructure in comparison to what is needed for static traffic models. Macroscopic approaches, however, are more similar to static models, regarding their level of aggregation and simplification of the road infrastructure. Such network loading approaches have been incorporated in static models, in order to take into account interactions between the links (Bundschuh et al., 2006b; Bliemer et al., 2012). However, this type of models has so far only been used for improved estimation of travel times on routes, but not for emissions estimation since their outputs cannot straightforward be applied to an emission model.

In this thesis, we choose to use an emission-based version of the model proposed by Bliemer et al., (2012). The queue propagation in this model, being based on the kinematic wave theory, is more realistically described compared to the method proposed by Bundschuh et al., (2006b).

\subsection{Quasi-dynamic network loading as a post-processing approach}

STAQ (Brederode et al., 2010; Leeuwen, 2011; Bliemer et al., 2012; Brederode et al., 2018) is a network loading model based on the LWR first-order traffic flow model. Although in LWR the transitions between traffic states are not described in detail and could be unrealistic regarding the rates of acceleration (Treiber and Kesting, 2013), shockwave positions are accurately described. The latter is more important for the needs of this study, since HBEFA emission factors are functions of speed and acceleration variations are implicitly modelled, being included in the associated driving cycle. A more complicated emission model, sensitive to acceleration, would require a better description 
of the state's transitions, for instance by a second-order traffic flow model. Consequently, considering the nature of HBEFA model, we use a first-order traffic flow model, assuming that infinite acceleration can happen instantaneously at the transitions, since that doesn't affect the emission estimates.

The network loading model STAQ is a quasi-dynamic model, being based on the dynamic eLTM model and considering two important static assumptions: demand is stationary and part of the demand is loaded instantaneously (see Section 2.2.2). The flow propagation is based on the simplified wave theory of Newell, (1993), using cumulative flows and the triangular fundamental diagram (Leeuwen, 2011; Bliemer et al., 2012). Nevertheless, according to the triangular fundamental diagram the speed is constant at the flowing regime, since there is linear relation between flow and density. Although constant speed at the flowing regime might not have a significant effect regarding the travel time, concerning an emission estimation analysis, those speed variations can be crucial. Specifically, in the case of HBEFA emission factors, the uncongested part of the fundamental diagram may represent both free flow and heavy situation. Therefore, the distinction between those two situations may be difficult using a linear density-flow relationship. In our study, we use a hyperbolic-linear fundamental diagram described in Work et al., 2010, where the speed at the flowing regime is not constant but depends on the density. The fundamental diagram parameters are estimated through calibration using the speed and flow counts.

The main output of STAQ model is average link travel times, calculated by the cumulative number of vehicles. However, by applying the STAQ algorithm it is also possible to keep track of the spatial position of the shock-wave whenever an event occurs. In our study, to make the model outputs suitable for emission estimation, we modified parts of the algorithm described in Brederode et al., (2010). The detailed description of this modified algorithm can be found in Appendix B. Using the queue spill-back information, we split each link into queuing and running space-time areas. Each area can correspond to different regime of the fundamental diagram and different traffic state $[\rho, Q, V]$.

Figure 5.1 illustrates the spatio-temporal evolution of shock-waves defining the transition between four different traffic states, for a network link, $a$. At an event time $\varepsilon^{1}$, a backward moving shock-wave with speed $w_{a}^{1 \rightarrow 2}$, hits the downstream end of link $a, a_{L}$, while a faster 


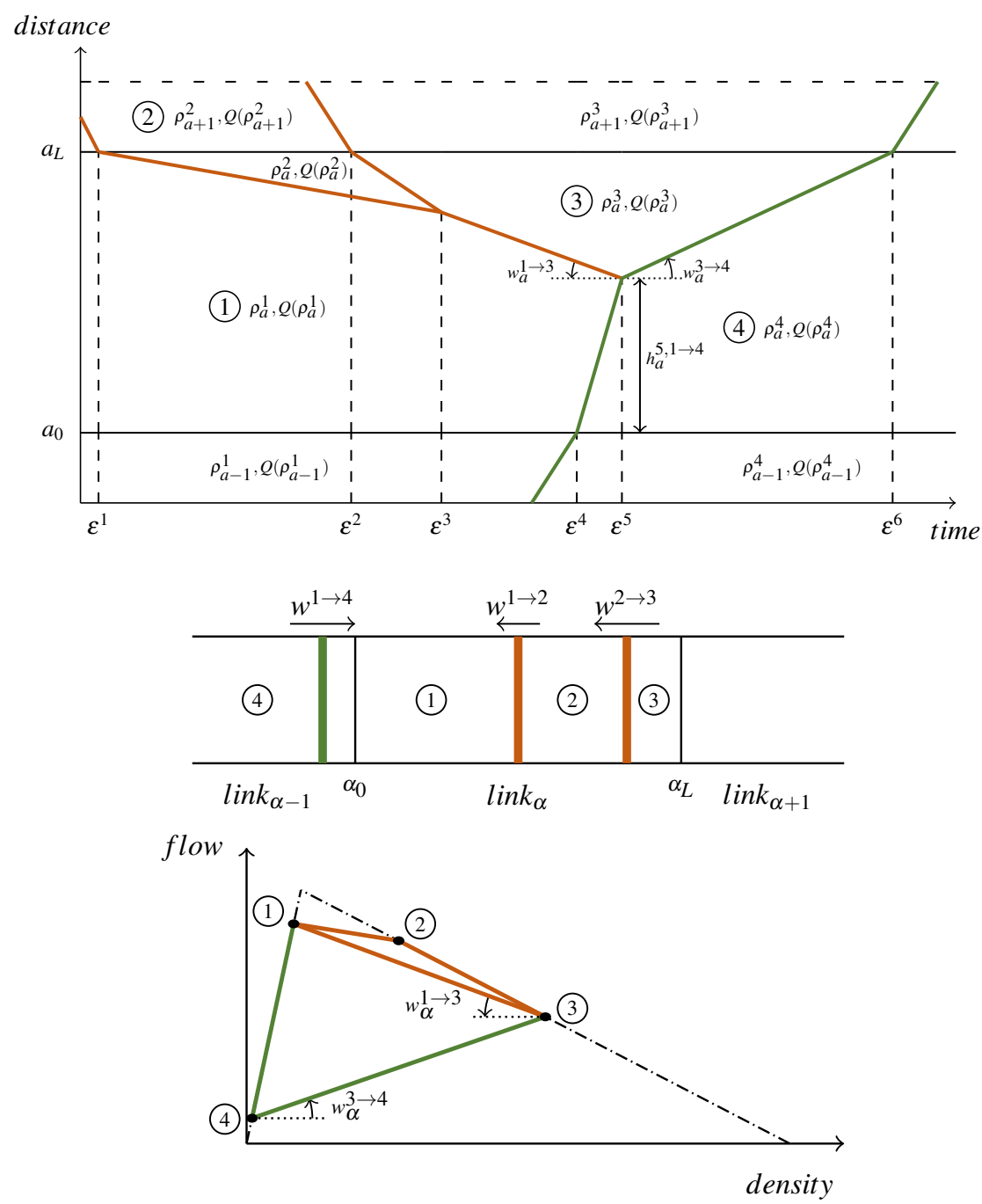

Figure 5.1: Spatial and temporal evolution of shock-waves. 
backward shock-wave with speed $w_{a}^{2 \rightarrow 3}$, hits the same point at time $\varepsilon^{2}$. The second wave will finally meet the first one, and a new event will occur at time $\varepsilon^{3}$. The new wave that will be formed from their merge, will then meet a forward moving wave at $\varepsilon^{5}$. The later had previously hit the upstream end $a_{0}$ at $\varepsilon^{4}$. Finally, at time $\varepsilon^{6}$, a new forward wave will reach the downstream end $a_{L}$. Figure 5.1, illustrates all the possible event types, as are described in Section 2.2.2. Since the algorithm is event-based, time is discretised based on the events and the number of algorithm steps equals to the number of events.

From the time-distance plot we can notice that the shock-waves divide a link into different smaller areas, each one of them associated with different traffic states. Particularly, in the case of Figure 5.1, the link $a$ is divided into four areas. Each area is related to a specific density $\rho$, flow $Q(\rho)$, as well as to a specific average speed $V(\rho)$. Since emission factors can be calculated as a function of average speed $V(\rho)$, each area is also associated with a different emission factor $e^{p}[V(\rho)]$, for every pollutant $p$.

Let $\mathcal{M}=\{1,2, \ldots, N-1\}$, where $N$ is the total number of events during the simulation period. Every link of the network, $a$, at each step $n$, is divided into $P_{a}^{n}$ parts depending on the number of shockwaves, $W_{p}^{n}$, traversing the link at the time of the $n t h$ event, $\varepsilon^{n}$. Let $\mathcal{P}_{a}^{n}=\left\{1,2, \ldots, P_{a}^{n}\right\}$ and $\mathcal{W}_{a}^{n}=\left\{1,2, \ldots, W_{a}^{n}\right\}$. The algorithm keeps track of the position, namely the distance from the downstream end of the link, $h_{a, j}^{n}$, and the shock-wave speed, $w_{a, j}^{n}$, of every shockwave, $j \in \mathcal{W}_{a}^{n}$. The algorithm keeps also track of the traffic state, $\left[\rho_{a, i}^{n}, Q_{a, i}^{n}, V_{a, i}^{n}\right]$ for each part of the link, $i \in \mathcal{P}_{a}^{n}$. Since STAQ is based on a first order model, the transitions between the different traffic states are linear in the space-time domain and they can be expressed through linear equations of the form $y_{1}=m \cdot y_{2}+b$. The slope $m$ is by definition equal to the shock-wave speed $w_{a, j}^{n}, j \in \mathcal{W}_{a}^{n}$ while the constant $b$ equals to $h_{a, j}^{n}-w_{a, j}^{n} \varepsilon^{n}, j \in \mathcal{W}_{a}^{n}$. Hence, the area in the space-time domain $R_{a, i}^{n}$ of its part $i \in \mathcal{P}_{a}^{n}$, which is defined between two shock-waves $j$ and $j+1, j \in \mathcal{W}_{a}^{n}$ and between two time steps $n, i+1$, can be computed as 


$$
\begin{aligned}
R_{a, i}^{n} & =\int_{\varepsilon^{n}}^{\varepsilon^{n+1}}\left(w_{a, j+1}^{n} \omega+h_{a, j+1}^{n}-w_{a, j+1}^{n} \varepsilon^{n}\right) d \omega \\
& -\int_{\varepsilon^{n}}^{\varepsilon^{n+1}}\left(w_{a, j}^{n} \omega+h_{a, j}^{n}-w_{a, j}^{n} \varepsilon^{n}\right) d \omega, \quad \forall i \in \mathcal{P}_{a}^{n}, \forall j \in \mathcal{W}_{a}^{n},
\end{aligned}
$$

where $w_{a, W_{a}^{n}+1}^{n}=0$ and $h_{a, W_{a}^{n}+1}^{n}=L_{a}$, for all $a \in \mathcal{A}$ and $n \in \mathcal{M}$. The area multiplied by the flow rate at the step $n, Q_{a, i}^{n} \cdot\left(\varepsilon^{n+1}-\varepsilon^{n}\right), i \in \mathcal{P}_{a}^{n}$, gives the vehicle kilometres travelled for the specific state. Finally, for each link $a$ the grams of each pollutant $p$ emitted between time $\varepsilon^{n}$ and $\varepsilon^{n+1}$, can be estimated as

$$
E_{a}^{n, p}=\sum_{i \in \mathcal{P}_{a}^{n}} R_{a, i}^{n} \cdot Q_{a, i}^{n} \cdot\left(\varepsilon^{n+1}-\varepsilon^{n}\right) \cdot e_{a}^{p}\left(V_{a, i}^{n}\right) .
$$

\subsection{Computational results}

\subsubsection{Case study}

Our hypothesis is that the application of a quasi-dynamic model as a post-processing technique could potentially reduce the emissions estimation errors introduced by static models. To test our hypothesis, we applied STAQ for the same Stockholm's test site (see Section 4.2.1) and the results expressed in ECEP terms (see Section 4.2.3), are illustrated in Figure 5.2.

As it is depicted in Figure 5.2 (b), the excess emissions are estimated upstream the bottleneck and the queues are propagated backwards. Therefore, in contrast to the static case (Figure 5.2 (c)), the higher excess emissions are located almost at the same positions as the ground-truth. Figure 5.2 demonstrates that post-processing the static assignment outputs, can actually lead to a more accurate spatial distribution of emissions. It is clear that the errors are now significantly reduced especially for the links upstream the bottleneck. In terms of total segment emissions, we also notice an improvement since the differences with the ground-truth now range from $0.23 \%$ to $1.05 \%$ depending on the pollutant. 
(a)

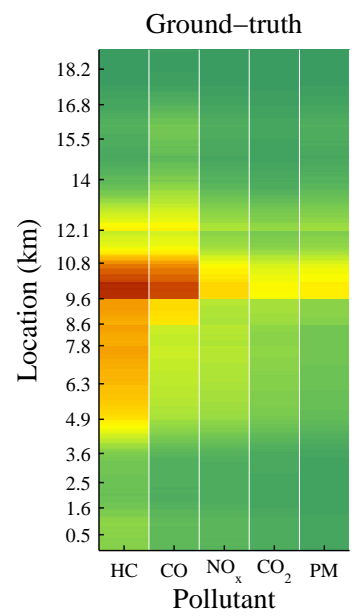

(b)

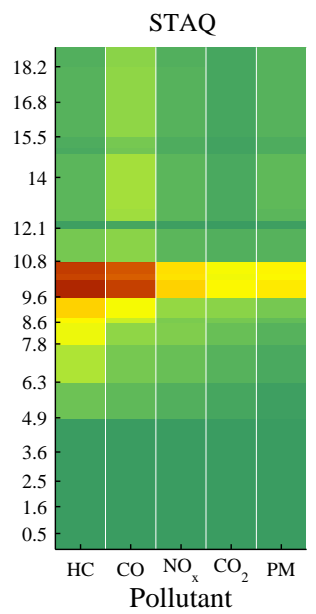

(c)

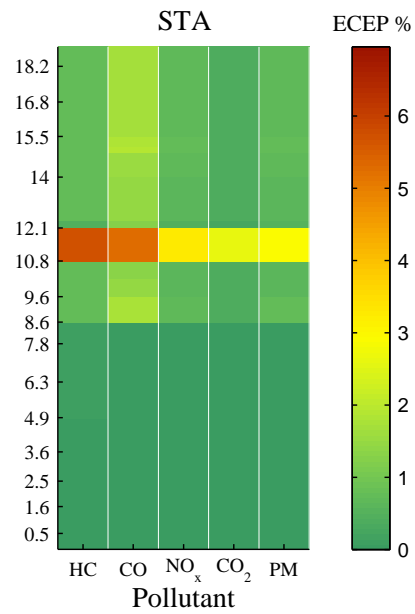

Figure 5.2: Average excess due to congestion emissions (ECEP) for (a) the ground-truth (b) static loading with queuing (c) static loading.

\subsubsection{Network effects}

In the previous subsection we demonstrate that post-processing of STA results can lead to more realistic emission estimates for a simple corridor network including one only bottleneck. In this section we evaluate the use of the STAQ post-processor considering a larger network with actual intersections where traffic is merged or diverged. The evaluation is performed by comparing the total network emissions as well as the spatial distribution of emissions that arise from the simple STA with the corresponding ones derived from the STAQ approach.

\section{Norrköping network}

Norrköping is a city in the province of Östergötland in eastern Sweden with a population of 137.000 inhabitants. Figure 5.3(a), illustrates the Norrköping network which is represented by 155 zones, forming $155 \times$ $155=24025$ O-D pairs, 513 nodes and 1344 links. The city centre, where the main congestion problems can be identified, is depicted in Figure 5.3 (b). The grey dashed line is associated with the connectors, the pseudo-links that connect the zones' centroids with the real nodes of the network. The demand between each O-D pair regards the 
(a)

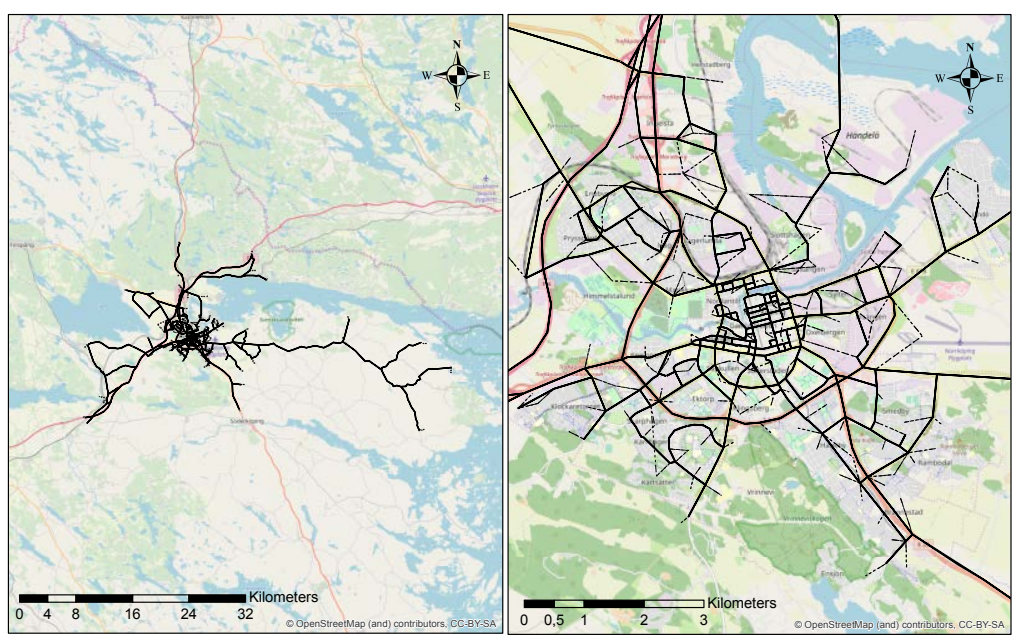

Figure 5.3: (a) Norrköping greater area network, (b) Norrköping's city centre. The real network's links are denoted by the black solid line while the connectors are by the grey dashed line.

number of trips made from private traffic during the peak hour for a typical day.

STAQ squeezing phase, given the path flows, blocks the paths containing a link where flow exceeds capacity through an incremental assignment. Therefore, this phase requires path flow information as input, i.e. the $f_{k}^{r s}$, meaning the part of the demand $f^{r s}$ that is assigned to every possible path $k$ between $r$ and $s$. For this reason, in order to solve the STA Problem (2.12) and equilibrate the network, we use a path-based algorithm, the Disaggregate Simplicial Decomposition (DSD) algorithm (Larsson and Patriksson, 1992).

The resulting optimal path shares, $f_{k}^{r s}$, with the help of the incidence matrix, $\delta$, lead to the equilibrated link flows, $x_{a}$, through Equation (2.12.d). We present the results of DSD algorithm in terms of volume to capacity ratio, V/C (Figure 5.4), since this is the most significant attribute determining the emission factors.

Then, for each link of the network, $a$, the emissions, $E_{a}^{p}$, in grams of pollutant $p$ emitted can be computed by Equation (4.1). Figure 5.5 illustrates the ECEP for the CO per vehicle and kilometre for the central roads of Norrköping. The width of the bars is analogous to the assigned flow. Therefore, the severity of those excess emissions 


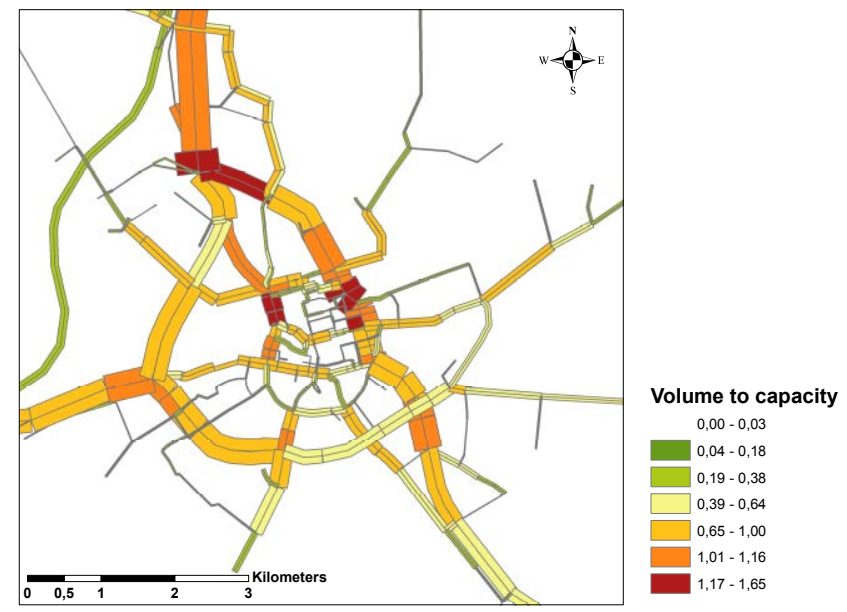

Figure 5.4: The resulting traffic flow pattern of STA presented as volume to capacity ratio for each link.

is analogous to the area of the rectangular representing each link (assigned flow $\times$ length).

We can notice high ECEP values concentrated at three different locations, where also the highest $\mathrm{V} / \mathrm{C}$ are observed (Figure 5.4). The links corresponding to these locations become activated bottlenecks during the peak hour, being the exclusive entrances to the city centre. STA assigns almost all the travel time delays in to those bottlenecks, neglecting the queues that may be formed at the upstream links. As we have highlighted in Section 4.1, from a travel time perspective, the spatial position of the delays is not of high importance, if the total path time is accurately predicted. Nevertheless, STA assigns also all the excess emissions created by the bottleneck at to the bottleneck links, while the upstream links remain unaffected. From an air-quality and exposure point of view the inaccurate spatial allocation of the excess emissions may lead to unreliable results.

For the same network we apply the STAQ model and the emissions are calculated according to the algorithm described in Appendix B. The path shares computed by the DSD are used as input to the squeezing phase. The demand is incrementally assigned to the paths in such a way that no link flow exceeds capacity. When a link flow reaches the capacity, every path containing this link is blocked at this point and all the downstream path links cannot accept any flow during the assignment of any next increment. In this way, when some 


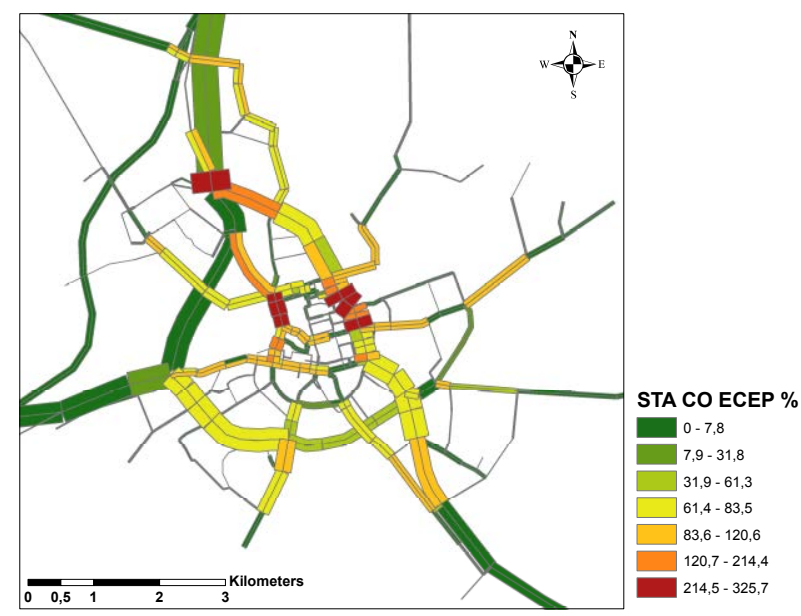

Figure 5.5: CO ECEP for the city centre links based on simple STA. The width of the bars is analogous to the assigned flow in each direction.

additional flow is to be assigned over a path that contain a blocked link, vertical queues are formed at the upstream end of the blocked link. In the Norrköping network, the demand is assigned through 24 increments and the results are illustrated in Figure 5.6. The radius of each circle denotes the length of the vertical queues in number of vehicles. The width of each bar is analogous to link inflow while the colour to the inflow to capacity ratio. Notice that after the squeezing phase there is no link where inflow exceeds capacity.

During the queuing phase, the vertical queues are propagated through the network based on an event-based algorithm (see Appendix B). The algorithm terminates when every vehicle has reached its destination. Applying the algorithm for the Norrköping network 437 events are occurred. Figure 5.7 illustrates the resulting ECEP for the CO. We can see a different pattern now, the excess due to congestion emissions are not concentrated at the bottleneck links. Instead, they are observed at the links upstream the bottlenecks due to of queue spill-back. Therefore, the emission rates based on STAQ model may be more appropriate for being an input to air-quality and exposure modelling. Regarding the total network emissions, the emissions based on STAQ are increased by $0.62 \%$ to $1.98 \%$ depending on the pollutant. 


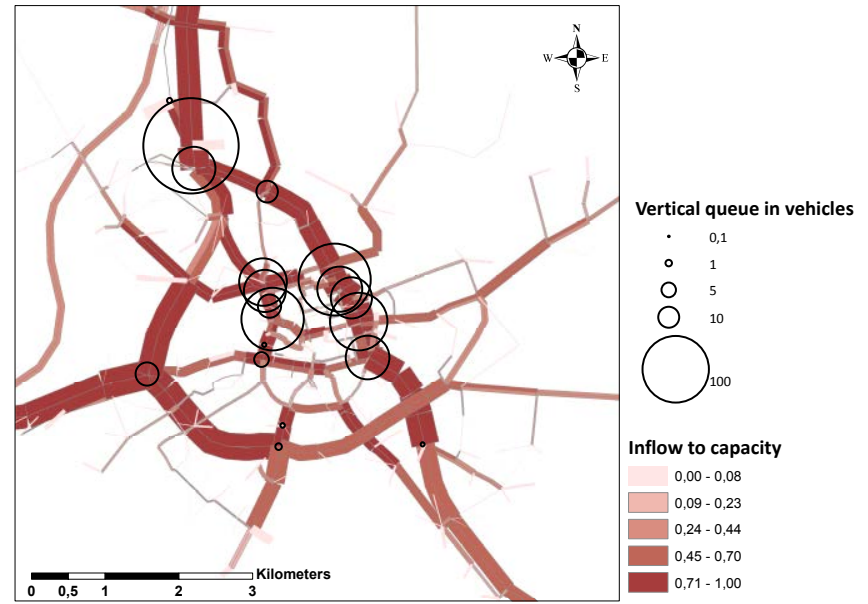

Figure 5.6: Squeezing phase results. The radius of each circle denotes the length of the vertical queues, while the colour of the bars is associated with link inflow.

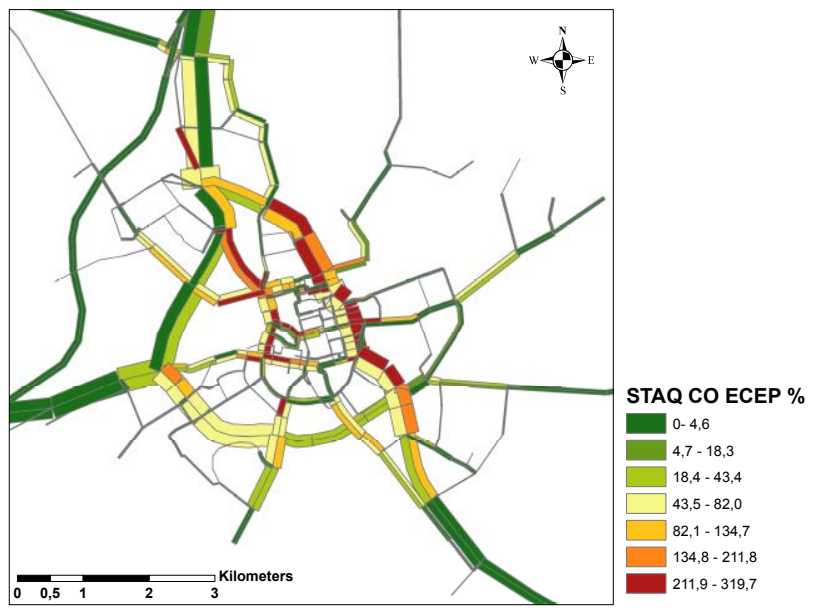

Figure 5.7: CO ECEP for the city centre links based on STAQ. The width of the bars is analogous to the assigned flow in each direction. 


\section{Exposure analysis}

Using STAQ instead of STA could affect the total emissions of a network, but the main differences lie on the spatial distribution of the excess due to congestion emissions. Our initial hypothesis is that the dissimilar, between STA and STAQ, way of modelling congestion may have effects on the applications of emission modelling. In this subsection we attempt to quantify these effects, focusing mainly on exposure modelling. Using a simplified exposure modelling framework, we calculate the number of people exposed to various emission rates, when these rates are estimated based on either STA or STAQ. Our methodology considers several assumptions since dispersion and exposure modelling are outside of the scope of this thesis. Our aim is to demonstrate, even by applying an oversimplified exposure model, that quasi-dynamic loading and queue spill-back does influence the number of people exposed to vehicular emissions.

Road traffic emissions are line emission sources. A line source is a one-dimensional emission source that exhibits a line type of geography. We assume that each line source has an area of influence, meaning that the people inside this area are exposed to the pollutant. This area is defined by a specific distance from the street. Since we apply static traffic modelling, resulting in one-hour average traffic variables, we also assume static population density during the analysis period. Having information on where people live, the number of people being inside the area of influence can be computed. An area of influence has been used in many proximity exposure analyses (Jerrett et al., 2005) and the width of this area varies depending on the pollutant and the application (Gilbert et al., 2003; Zhu et al., 2002). In this study, the area of influence is set to $300 \mathrm{~m}$.

Line emission sources are associated with one-dimensional pollutant emissions, expressed in mass per space and time unit. Dispersion models describe the propagation of the mass over the threedimensional space and time, resulting in concentrations per time unit. The mass propagation, which is based on the wind speed and other meteorological attributes, should ensure the mass conservation. In our static case, we don't take into account any dynamic dispersion. Additionally, a two-dimensional space domain is considered, and finally our results are expressed in densities $\left(\mathrm{g} / \mathrm{m}^{2}\right)$, describing average conditions for the whole analysis period.

Since the pollutant concentrations close to the source are higher 
(Zhu et al., 2002), the pollutant densities are also not homogeneous alongside the area of influence. Thus, we assume that the density is diminished while you are moving away from the source. To be consisted with the mass conservation, we model this density diminution with the distance from the source, using a kernel density function (Silverman, 1986). Kernel functions are used in many Lagrangian particle dispersion models to reconstruct the concentrations. The advantages of using kernel functions instead of simple box models to reconstruct the concentrations are highlighted in Haan, (1999) and Vitali et al., (2006). The two-dimensional space is discretised in square cells with a width of $\tau$. At each cell, the mass, $m_{p}(x, y)$, of pollutant $p$ in grams is computed by summing up the parts of all the line sources that coincide within the cell. Each cell can be assumed as microenvironment, being a chunk of air space with homogeneous pollutant density. The kernel density, $\hat{f}(x, y)$, is computed as

$$
\hat{f}_{p}(x, y)=\sum_{i=1}^{n} \frac{m_{p}\left(x_{i}, y_{i}\right)}{\tilde{h}^{2}} K\left(\frac{d_{i}}{\tilde{h}}\right),
$$

where $\tilde{h}$ is the search radius bandwidth, $n$ the number of the cells inside the circle with $(x, y)$ as centre and radius equal to $\tilde{h}, d_{i}$ is the geographical distance between $\left(x_{i}, y_{i}\right)$ and $(x, y)$, and $K(u)$ is a kernel function satisfying the two following requirements,

$$
\begin{aligned}
K(u) & \geq 0, \forall u \in \Re, \\
\int_{\Re} K(u) & =1,
\end{aligned}
$$

the last requirement ensures the mass conservation. In this study, the search radius bandwidth is equal to the width of influence area, $300 \mathrm{~m}$, and the cell width, $\tau$, is $10 \mathrm{~m}$. Regarding the kernel function, $K(u)$, we use the quartic one,

$$
K(u)=\left\{\begin{array}{cc}
\frac{15}{16}\left(1-u^{2}\right)^{2} & \text { if }|u| \leq 1 \\
0 & \text { else }
\end{array} .\right.
$$

Figure 5.8 illustrates the resulting $\mathrm{CO}$ densities for each cell in grams per square metre for both STA and STAQ approach. According to the STA case, the highest densities are observed close to the bottleneck links, while according to STAQ, the spatial distribution of densities is smoother due to the propagation of the queues. 

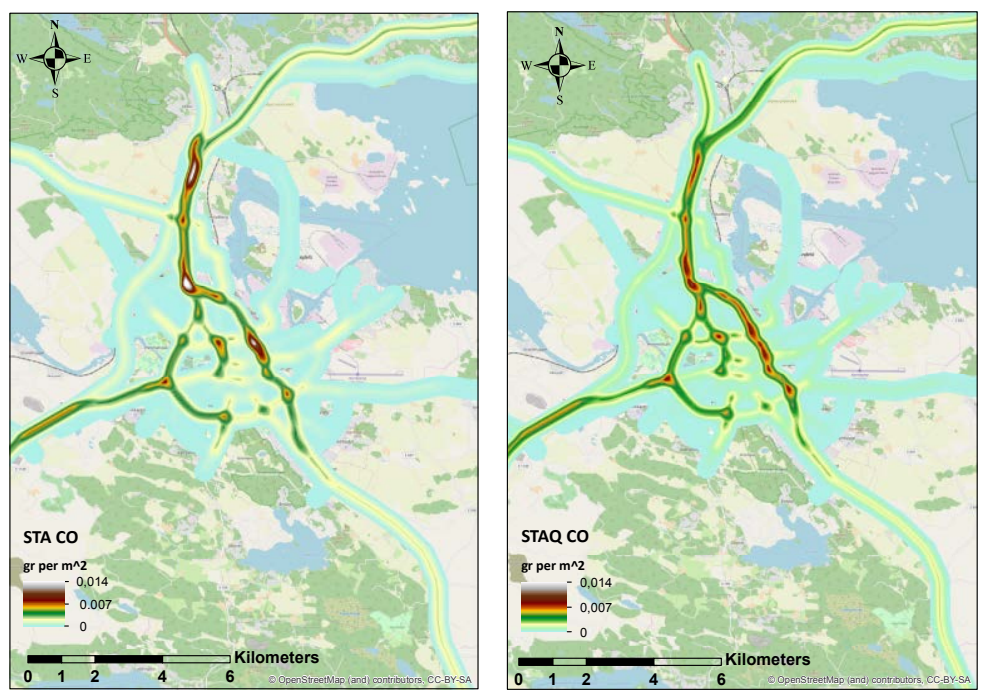

Figure 5.8: CO density in $\mathrm{gr} / \mathrm{m}^{2}$ based on STA (left-hand side) and STAQ (right-hand side).

Having information on where people live, we construct a population density grid, in number of people $/ \mathrm{m}^{2}$, with the same cell resolution. In order to compare the performance of STA and STAQ with respect to exposure, we introduce the term People Grams Exposure (PGE) expressed in $\left(g r / m^{2}\right) \times\left(\right.$ people $\left./ \mathrm{m}^{2}\right)$. We compute the corresponding PGE grid by multiplying the pollutants density by the population density for each cell.

Although term PGE doesn't have any physical meaning, it is a useful tool to identify the locations which are associated with high exposure rates, meaning locations where a large number of people are exposed to high pollutants densities. In addition, PGE helps us to illustrate and evaluate the effects that the two different approaches STA and STAQ have on exposure analyses.

To compare these two different approaches, we calculate the relative differences between the two grids, $P G E_{S T A}^{p}$ and $P G E_{S T A Q}^{p}$ for each pollutant $p$. The statistics (at a cell level) of these differences are presented in Table 5.1. The last row of Table 5.1 illustrates the mean (at the level of a meter) relative difference between the two approaches for the emission rates, $E^{p}$, estimated in grams per meter.

We notice that the PGE differences vary depending on the pollutant and for some cells they can reach the $113 \%$. Moreover, an 
Table 5.1: Min, Max, Mean and Standard Deviation of the relative PGE difference between the two different methods of deriving the traffic data. The last row illustrates the mean relative difference between the two approaches for the emission rates, E.

\begin{tabular}{lccccc}
\hline PGE, STA-STAQ $\%$ & $\mathrm{HC}$ & $\mathrm{CO}$ & $\mathrm{NO}_{\mathrm{x}}$ & $\mathrm{CO}_{2}$ & $\mathrm{PM}$ \\
\hline Min & -113.37 & -97.15 & -63.48 & -53.11 & -55.75 \\
Max & 65.31 & 54.93 & 49.45 & 46.21 & 46.63 \\
Mean & -5.7 & -1.30 & -2.02 & -2.30 & -1.43 \\
Std Dev & 20.34 & 15.03 & 11.15 & 9.78 & 9.90 \\
\hline E, STA-STAQ \% & $\mathrm{HC}$ & $\mathrm{CO}$ & $\mathrm{NO}_{\mathrm{x}}$ & $\mathrm{CO}_{2}$ & $\mathrm{PM}$ \\
\hline Mean & -2.45 & 1.45 & -0.53 & -1.36 & -0.41 \\
\hline
\end{tabular}

interesting finding is that the mean differences in terms of exposure are higher than the corresponding emission rates mean differences. Therefore, quasi-dynamic network loading can influence the emission rates, but can have a stronger, non-linear, effect on applications of emissions such as exposure modelling. Figure 5.9 illustrates the estimated PGE for the HC. HC is the pollutant for which we observe the highest differences between STA and STAQ, since it is the most sensitive to speed variations pollutant. On one hand, PGE based on STA is higher for cells that are located close to a bottleneck. On the other hand, for cells located close to an upstream of a bottleneck link, PGE based on STAQ is higher.

Finally, we can conclude that queue spill-back considered in STAQ affects the exposure modelling. Hence, our initial hypothesis is verified. However, we draw this conclusion by applying a simplified exposure model. The main limitations of our underlying method regard the neglect of wind speed and direction as well as of any other background pollution. In addition, we make the not so realistic assumption that people are present in their homes during the peak hour and that every pollutant has the same area of influence. It would be interesting for the future to follow a more realistic dispersion and exposure modelling framework in order to identify the differences between the STA and STAQ approach. One more interesting topic for future research is to investigate if these differences can have an effect on the damage cost of each pollutant and if this effect can be significant with respect to economic analyses such as Cost-Benefit Analyses. 

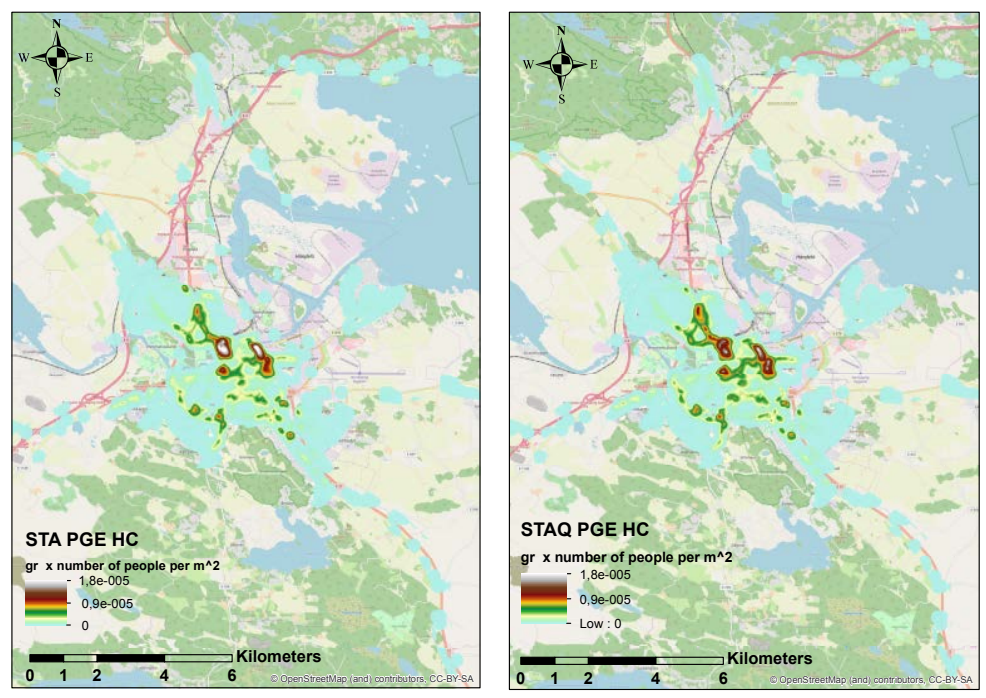

Figure 5.9: HC PGE in $\left(g r / m^{2}\right) \times\left(\right.$ people $\left./ m^{2}\right)$ based on STA (left-hand side) and STAQ (right-hand side).

\subsection{Discussion}

As we show in the previous chapter, STA can lead to inaccurate rates and spatial distribution of emissions, affecting applications of emissions such as dispersion and exposure modelling. In this chapter we suggest the use of a quasi-dynamic network loading model as a post-processor of STA results. Our hypothesis is that quasidynamic network loading, taking into account spill-back effects, can lead to more reliable emission rates but mainly to more reliable spatial distribution of these rates. To test our hypothesis, we apply a quasi-dynamic loading model, STAQ for our case study road segment. Compared to the simple static case, post-processing leads to reduced errors regarding the spatial distribution of emission as well as the total emission rates. The excess due to congestion emissions are now modelled upstream bottleneck as they are observed in reality. Knowing that quasi-dynamic loading has a better performance in a corridor network, we test the same post-processing approach for a larger, more realistic network. The results of the comparison between STA and STAQ show that, although the differences in terms of total network emissions are not so high, the spatial distribution of emissions is substantially changed. Considering the location specific 
effects of emissions, dissimilar location of the excess emissions could influence exposure and air quality modelling.

We should note here that we use a quasi-dynamic model, STAQ, as a post-processor, only for the network loading phase and only for emission estimation purposes. Route choice is exogenous to the model, it relies on route travel times derived from STA. We assume that travellers choose their routes trying to minimize their travel time according to a simple VDF, without being influenced by the quasi-dynamic loading. Therefore, there is an inconsistency regarding the way that travel times and emissions are estimated. By applying STAQ, we can also obtain information on the average speed of each link. Thus, the average link travel times and consequently the route travel times can be estimated once the network loading is performed. Although the network is equilibrated with respect to STA travel times, is not equilibrated in terms of STAQ link travel times. Using an iterative algorithm like the corresponding ones used on DTA, STAQ route travel times can be equilibrated. Nevertheless, similarly to the DTA approaches, the uniqueness of the solution is not guaranteed.

Using STAQ only as a post-processor, the total network emissions or route emissions are not significantly changed. Thus, single quasidynamic loading with a predefined route choice can be adequate for emission estimation purposes. In the thesis, we suggest the use of this approach, only to obtain a more accurate spatial distribution of emissions. The investigation of how suitable STAQ is to be used in any other planning purpose where route travel time matters, is outside of the scope of this thesis. 


\section{Chapter 6}

\section{Conclusions and Future research}

\subsection{Conclusions}

Since road emissions are an important contributor to the total emissions of the main air pollutants, their estimation should be accurate in order to evaluate air pollution mitigation strategies. Traffic emission models are commonly used to estimate the road traffic emissions, by combing traffic data with some other factors such as vehicle fleet composition. The required input traffic data can be derived from either traffic models or sensor measurements. For large scale emission estimation analyses, the derivation of traffic data usually relies on static traffic models or cross-sectional sensor measurements. However, both approaches consider some assumptions regarding the spatio-temporal resolution of traffic variables and the variations of traffic conditions. The overall aim of the thesis is to identify, quantify and finally reduce the potential errors that the assumptions above introduce in an emission estimation analysis.

Firstly, in the case where cross-sectional sensor measurements are used to generate the input to emission models, the main errors that we identify are associated with the traditional approaches of extrapolating the measurements in time and space. For this reason we suggest the use of a more detailed traffic state estimator, the ASM. ASM provides a more detailed description of traffic by capturing the spatio-temporal variations and may lead to more accurate emission 
estimates. Therefore, the answer to the first research question is that traditional approaches of extrapolating traffic measurements in time and space do lead to less reliable emission data compared to ASM. However, the level of reliability depends on the investigated pollutant and the potential use of emission data. On one hand, since the differences between the traditional approaches and ASM in terms of annual emissions are relatively small, traditional approaches can be used more efficiently for emission inventories applications and pollutants such as $\mathrm{CO}_{2}$. On the other hand, their use may be inappropriate for sensitive to the spatio-temporal variations applications such as dispersion and exposure modelling and pollutants with local effects such as $\mathrm{CO}$ and $\mathrm{NO}_{\mathrm{x}}$.

Concerning the case where static traffic models are used to generate inputs to emission models, we distinguish two types of emission estimation errors, giving an answer to research Q2. Errors due to spatio-temporal aggregation and errors due to static loading. The first type of errors can be efficiently limited by using a continuous emission model. Regarding the second type of errors, the inability of STA models to describe dynamic traffic flow phenomena leads to inaccurate location of the excess due to congestion emissions. Excess emissions are estimated at the bottleneck link instead of upstream the bottleneck as they are observed in reality.

Considering the above inabilities of static models, we suggest and evaluate the post-processing of STA outputs. We notice that postprocessing of static modelling using quasi-dynamic loading leads to more accurate emission estimation. This accuracy relies mainly on the location of excess due to congestion emissions. In terms of total network emissions, the differences derived from the post-processing are not so high. Therefore, we can give an answer to research Q3, similar to the one given for research Q1. For large-scale or longterm emission analyses, static traffic models can sufficiently be used to provide the input traffic data. Those analyses can regard emission inventories or $\mathrm{CO}_{2}$ effects. Contrary, for more sensitive to spatiotemporal variations of traffic conditions applications such as exposure and exposure modelling, the accurate estimation of the congestion's location is significant. In such cases, post-processing of static results becomes a crucial step during the emission estimation process. 


\subsection{Future research}

In the thesis we show that post-processing using quasi-dynamic loading leads to an improved spatial distribution of the emitted pollutants. Considering the fact that many applications of emissions data are location sensitive, we conclude that these applications may be affected by post-processing. We draw this conclusion assuming that emission rates have a direct effect on air quality. It would be interesting in the future to try to quantify this effect and evaluate the significance of it.

The emission estimation methodology proposed in the thesis, can be used in future studies to generate inputs for dispersion modelling. In this way, the actual differences between static and quasi-dynamic modelling in terms of pollutants concentrations can be investigated. Future studies could also investigate the association between the differences in the spatial distribution of emissions due to post-processing with the damage cost of each pollutant. In addition, the significance of these spatial distribution differences can be investigated when economic analyses are performed.

In the thesis we demonstrate that quasi-dynamic modelling can lead to more accurate emission estimates, mainly regarding the spatial distribution, compared to the simple static modelling. However, the performance of quasi-dynamic modelling compared to an advanced dynamic model has not yet been investigated. Future research could evaluate the accuracy of this quasi-dynamic post-processor on a network level, having some more advanced DTA models as comparison.

Regarding the case where sensors data is used to generate inputs for an emission estimation, the thesis focuses on Eulerian type of data. However, alternative sensors providing Lagrangian data, such as vehicle probe and Bluetooth data, are becoming more and more available today. Although this type of data is less costly to collect, it only provides travel time data. Traffic flows, which is the most significant input for emission estimation, have to be estimated based on the travel times. Different approaches of estimating traffic flows from travel times may affect the emission estimation. The development of such approaches and the investigation of their impacts on emission modelling provide a good starting point for discussion and further research. 

Appendices 

Appendix A

Vehicle fleet

composition in Sweden

and HBEFA emission

factors 
Table A.1: The considered vehicle composition for passenger cars petrol PC-P and PC-C according to the European standards for Light Duty Vehicles.

\begin{tabular}{ll}
\hline Vehicle class & \% of the total fleet \\
\hline PC-P-Euro-0 & 0.93 \\
PC-P-Euro-1 & 1.14 \\
PC-P-Euro-2 & 7.63 \\
PC-P-Euro-3 & 4.25 \\
PC-P-Euro-4 & 24.25 \\
PC-P-Euro-5 & 9.09 \\
PC-P-Euro-6 & 0.25 \\
PC-Alternative Fuel & 14.55 \\
PC-C-Euro-0 & 0.03 \\
PC-C-Euro-1 & 0.06 \\
PC-C-Euro-2 & 0.37 \\
PC-C-Euro-3 & 1.11 \\
PC-C-Euro-4 & 12.35 \\
PC-C-Euro-5 & 23.00 \\
PC-C-Euro-6 & 0.99 \\
\hline
\end{tabular}


Table A.2: The HBEFA emission factors used for this study in grams of pollutant emitted per kilometre for five pollutants ( $\mathrm{HC}, \mathrm{CO}, \mathrm{NO}_{\mathrm{x}}, \mathrm{CO}_{2}, \mathrm{PM}$ ), four traffic situations (Free flow, Heavy, Saturated, Stop and go) and for two road types, urban motorway with a speed limit of $70 \mathrm{~km} / \mathrm{h}$ (URB/MW/70) and urban motorway with a speed limit of $90 \mathrm{~km} / h$ (URB/MW/90).

\begin{tabular}{llll}
\hline Pollutant & Traffic situation & URB/MW/70 & URB/MW/90 \\
\hline $\mathrm{HC}$ & Free flow & 0.016 & 0.016 \\
& Heavy & 0.022 & 0.016 \\
& Saturated & 0.022 & 0.021 \\
$\mathrm{CO}$ & Stop and go & 0.073 & 0.057 \\
& Free flow & 0.167 & 0.278 \\
& Heavy & 0.337 & 0.219 \\
& Saturated & 0.261 & 0.336 \\
$\mathrm{NO}_{\mathrm{x}}$ & Stop and go & 0.711 & 0.622 \\
& Free flow & 0.174 & 0.194 \\
& Heavy & 0.240 & 0.189 \\
& Saturated & 0.223 & 0.220 \\
$\mathrm{CO}_{2}$ & Stop and go & 0.524 & 0.406 \\
& Free flow & 109.95 & 127.98 \\
& Heavy & 132.88 & 121.50 \\
& Saturated & 133.30 & 132.69 \\
$\mathrm{PM}^{*}$ & Stop and go & 290.80 & 229.54 \\
& Free flow & 0.0027 & 0.0037 \\
& Heavy & 0.0038 & 0.0032 \\
& Saturated & 0.0034 & 0.0037 \\
& Stop and go & 0.0075 & 0.0058 \\
\hline
\end{tabular}



Appendix B

Description of the

modified STAQ

algorithm 


\section{B.1 Squeezing phase}

Solving the problem 2.12 will result into equilibrated route flows $f_{k}^{r s}$ with the link flows $x_{a}=\sum_{r \in \mathcal{R}} \sum_{s \in \mathcal{S}} \sum_{k \in \mathcal{K}^{r s}} f_{k}^{r s} \delta_{a, k}^{r s}, \forall r \in \mathcal{R}, s \in \mathcal{S}$, $a \in \mathcal{A}$ and $k \in \mathcal{K}^{r s}$.

\section{Initialization}

- For each origin-destination pair $r s$, determine a set of sets $\mathcal{D}^{r s}$, where each set $\mathcal{D}_{j}^{r s, k}$ contains the consecutive links $j$ of the route $k \in \mathcal{K}^{r s}$.

- Determine for each link $a$, the $\mathrm{V} / \mathrm{C}$ ratios $\xi_{a}=x_{a} / C_{a}$.

- Determine the set of potential/candidate blocking links $\mathcal{B}, \mathcal{B}=$ $\left\{a \in \mathcal{A} \mid \xi_{a}>1\right\}$.

- Determine the set of potential blocked routes for each origin destination pair $r s, \mathcal{L}^{r s}, \mathcal{L}^{r s}=\left\{k \in \mathcal{K}^{r s} \mid \mathcal{D}^{r s, k} \cap \mathcal{B} \neq \emptyset\right\}$.

- Determine the set of routes that are not blocked $\overline{\mathcal{L}}^{r s}, \overline{\mathcal{L}}^{r s}=$ $\mathcal{K}^{r s} \backslash \mathcal{L}^{r s}$.

- Set $i:=0$.

- Find the set the most restrictive $\operatorname{link}(\mathrm{s}), \tilde{\mathcal{A}}=\left\{a \in A \mid \xi_{a}^{i}=\right.$ $\left.\max _{a \in \mathcal{B}}\left\{\xi_{a}\right\}\right\}$.

- Assign all the route flows that are not blocked and determine the flow at the upstream end of each link $a, q_{a}^{i n, i}$

$$
q_{a}^{i n, i}=\sum_{r \in \mathcal{R}} \sum_{s \in \mathcal{S}} \sum_{k \in \overline{\mathcal{L}}^{r s}} f_{k}^{r s} \delta_{a, k}^{r s}
$$

(static assumption of instantaneous network loading).

- Set the flow at the downstream end of link $a, q_{a}^{\text {out }, i}, q_{a}^{\text {out }, i}=$ $q_{a}^{i n, i} \forall a \in \mathcal{A}$.

First increment

- Set the first increment $\theta^{i}=\frac{1}{\xi_{a}^{i}}, \forall a \in \tilde{\mathcal{A}}$.

- Set the inflow of each link

$$
q_{a}^{i n, i+1}=q_{a}^{i n, i}+\sum_{r \in \mathcal{R}} \sum_{s \in \mathcal{S}} \sum_{k \in \mathcal{L}^{r s}} f_{k}^{r, s} \delta_{a, k}^{r, s} \theta^{i}
$$


so that the most restricted $\operatorname{link}(\mathrm{s})$ will have inflow equal to capacity.

- Since no outflow is blocked yet, set the outflow equal to the inflow $q_{a}^{\text {out }, i+1}=q_{a}^{\text {in, } i+1} \forall a \in \mathcal{A}$.

- Determine a set of links $\tilde{\mathcal{B}}$ which includes the current/actual blocking $\operatorname{link}(\mathrm{s}), \tilde{\mathcal{B}}=\left\{a \in \mathcal{B} \mid q_{a}^{i n, i} / C_{a}=1\right\}$.

- Determine a set of routes $\tilde{\mathcal{K}}^{r s, b}$ which contain the routes that include the actual blocking link $b, \tilde{\mathcal{K}}^{r s, b}=\left\{k \in \mathcal{K}^{r s} \mid \mathcal{D}^{r s, k} \cap b \neq\right.$ $\emptyset\} \forall b \in \tilde{\mathcal{B}}$.

- Create two new incident matrices $\tilde{\delta}_{a, k}^{r s, i n}=\delta_{a, k}^{r s}$ and $\tilde{\delta}_{a, k}^{r s, i n}=\delta_{a, k}^{r s}$ $\forall r \in \mathcal{R}, s \in \mathcal{S}, a \in A$ and $k \in \mathcal{K}^{r s}$, to separate the inflows and the outflows.

Main loop: incremental loading

1 Set $\mathrm{i}:=\mathrm{i}+1$.

2 Determine, for each route $k \in \tilde{\mathcal{K}}^{r s, b}$, the position, $p^{b, k}$ of each actual blocking $\operatorname{link}(\mathrm{s}) b \in \tilde{\mathcal{B}}$, such as $\mathcal{D}_{p^{b, k}}^{r s, k}=b$.

3 For each blocking link $b \in \tilde{\mathcal{B}}$ and for each route $k \in \tilde{\mathcal{K}}^{r s, b}$ determine a set of links $\mathcal{O}^{r s, k, b}$ which contain all the links downstream the actual blocking $\operatorname{link}(\mathrm{s}) b, \mathcal{O}^{r s, k, b}=\left\{\mathcal{D}_{j}^{r s, k} \mid j>p^{b, k}\right\}$.

4 For each blocking link $b \in \tilde{\mathcal{B}}$ and for each route $k \in \tilde{\mathcal{K}}^{r s, b}$ determine the most upstream link of the actual blocking link $b$, $u^{k, b}=\mathcal{D}_{p^{b, k}-1}^{r s, k}$.

5 Block the routes at the upstream end of the blocking $\operatorname{link}(\mathrm{s})$ $b \in \tilde{\mathcal{B}}$, by setting $\tilde{\delta}_{a, k}^{r s, i n}=0$ and $\tilde{\delta}_{a, k}^{r s, o u t}=0 \forall k \in \tilde{\mathcal{K}}^{r s, b}, \forall a \in$ $\tilde{\mathcal{B}} \cup \mathcal{O}^{r s, k, b} \forall b \in \tilde{\mathcal{B}}$.

6 Block the routes at the downstream end of link $b$, by setting $\tilde{\delta}_{u^{k}, k}^{r s, o u t}=0$.

7 Update the set of the candidate blocking links removing the actual blocking links of the previous step $\mathcal{B}=\mathcal{B} \backslash \tilde{\mathcal{B}}$.

8 Update the $\xi$ for each blocking link, $\xi_{a}^{i+1}=q_{a}^{i n, i+1} / C_{a} \forall a \in \mathcal{B}$.

9 Update the set of actual blocking links, $\tilde{\mathcal{B}}=\left\{a \in \mathcal{B} \mid \xi_{a}^{i+1}=1\right\}$. 
10 Determine the new increment

$$
\theta^{i}=\theta^{i-1} \cdot \min _{a \in \mathcal{B}}\left\{\frac{1-\xi_{a}^{i}}{\xi_{a}^{i}-\xi_{a}^{i-1}}\right\} .
$$

11 Update the inflows

$$
q_{a}^{i n, i+1}=q_{a}^{i n, i}+\sum_{r \in \mathcal{R}} \sum_{s \in \mathcal{S}} \sum_{k \in \mathcal{L}^{r s}} f_{k}^{r, s} \tilde{\delta}_{a, k}^{r s, i n} \theta^{i}
$$

12 Update the outflows

$$
q_{a}^{\text {out }, i+1}=q_{a}^{\text {out }, i}+\sum_{r \in \mathcal{R}} \sum_{s \in \mathcal{S}} \sum_{k \in \mathcal{L}^{r s}} f_{k}^{r, s} \tilde{\delta}_{a, k}^{r s, \text { out }} \theta^{i} .
$$

13 Update the set of routes $\tilde{\mathcal{K}}^{r s, b}=\left\{k \in \mathcal{K}^{r s} \mid \mathcal{D}^{r s, k} \cap b \neq \emptyset\right\} \forall b \in \tilde{\mathcal{B}}$.

14 If $\sum_{i} \theta^{i}=1$, then stop. Otherwise, return to Step 1.

\section{B.2 Queuing phase}

\section{Initialization}

- Set $q_{a}^{\text {in, } 0}=q_{a}^{\text {in,end }}$ and $q_{a}^{\text {out }, 0}=q_{a}^{\text {out,end }} \forall a \in \mathcal{A}$, where $q_{a}^{\text {in,end }}$ and $q_{a}^{\text {out }, \text { end }}$ are the final inflows and outflows resulting from the squeezing phase.

- Determine the set of links $\mathcal{C}$, including the links where the inflow equals to the capacity $\mathcal{C}=\left\{a \in \mathcal{A} \mid g_{a}^{\text {in, }}=C_{a}\right\}$.

- Determine the set of links $\mathcal{Q}$, including the links where the inflow is greater than the outflow, $\mathcal{Q}=\left\{a \in \mathcal{A} \mid g_{a}^{\text {in, }, 0}>g_{a}^{\text {out }, 0}\right\}$.

- Set the densities at the start and end node of each link,

$$
\begin{aligned}
k_{a}^{\text {in, } 0} & =k_{a}\left(q_{a}^{\text {in }, 0} \mid \text { uncongested }\right) \forall a \in \mathcal{A} \backslash \mathcal{C} \\
k_{a}^{\text {in, } 0} & =k_{a}\left(q_{a}^{\text {in, }, 0} \mid \text { congested }\right) \forall a \in \mathcal{C} \\
k_{a}^{\text {out }, 0} & =k_{a}\left(q_{a}^{\text {out }, 0} \mid \text { uncongested }\right) \forall a \in \mathcal{A} \backslash(\mathcal{C} \cup \mathcal{Q}) \\
k_{a}^{\text {out }, 0} & =k_{a}\left(q_{a}^{\text {out }, 0} \mid \text { congested }\right) \forall a \in \mathcal{C} \cup \mathcal{Q},
\end{aligned}
$$


where according to the linear-hyperbolic fundamental diagram $k_{a}\left(q_{a} \mid\right.$ uncongested $)=\frac{k_{j, a}\left(u_{0, a}-\sqrt{-u_{0, a}\left(4 q_{a}-k_{j, a} u_{0, a}\right) / k_{j, a}}\right)}{2 u_{0, a}}$, and

$$
k_{a}\left(q_{a} \mid \text { congested }\right)=\frac{-q_{a}-k_{j, a} \cdot \omega_{a}}{\omega_{a}},
$$

where $k_{j, a}$ is the jam density, $u_{0, a}$ the free flow speed and $\omega_{a}$ the speed of a kinematic wave $\forall a \in \mathcal{A}$.

- Set the first event time $\varepsilon^{0}$ equal to zero $\varepsilon^{0}=0$.

- Set $n:=0$;

- For each link $a \in \mathcal{A}$ determine the number of the shock-waves traversing the link at the time of the event $\varepsilon^{n}, W_{a}$, and the set $\mathcal{W}_{a}=\left\{1,2, \ldots, W_{a}\right\}$.

Set

$$
\begin{array}{ll}
W_{a}=1 & \forall a \in \mathcal{Q} \\
W_{a}=0 & \forall a \in \mathcal{A} \backslash \mathcal{Q} .
\end{array}
$$

- For each link $a \in \mathcal{A}$ determine the number of the parts that the link is divided by the shock-waves at the time of the event $\varepsilon^{n}$, $P_{a}$, and the set $\mathcal{P}_{a}^{n}=\left\{1,2, \ldots, P_{a}\right\}$.

Set

$$
P_{a}=W_{a}+1 \quad a \in \mathcal{A} .
$$

- Each part of link $a \in \mathcal{A}, i \in \mathcal{P}_{a}$, is associated with an average flow $Q_{a, i}$, density $K_{a, i}$, speed $V_{a, i}$ and length $l_{a, i}^{n}$.

Set:

$$
\begin{aligned}
Q_{a, 1} & =q_{a}^{i n, 0}, K_{a, 1}=k_{a}^{i n, 0} \text { and } l_{a, 1}^{0}=L_{a} \quad \forall a \in \mathcal{A} \\
Q_{a, 2} & =q_{a}^{\text {out }, 0} \text { and } K_{a, 2}=k_{a}^{\text {out }, 0} \text { and } l_{a, 2}^{0}=0 \quad \forall a \in \mathcal{Q} \\
V_{a, i} & =Q_{a, i} / K_{a, i} \quad \forall i \in \mathcal{P}_{a}, \forall a \in \mathcal{A} .
\end{aligned}
$$

- For each link $a \in \mathcal{Q}$ determine the propagation velocity of shock fronts or the shock-wave speed

$$
w_{a, 1}=\frac{Q_{a, 2}-Q_{a, 1}}{K_{a, 2}-K_{a, 1}}
$$


- Find the candidate times for the first event $\epsilon_{a}=L_{a} / w_{a, 1} \forall a \in$ $\mathcal{Q}$.

- Set the first event time to $\epsilon^{1}=\min _{a \in \mathcal{Q}} \epsilon_{a}$ and find the associated with the first event $\operatorname{link}(\mathrm{s}) b, b=\left\{a \in \mathcal{Q} \mid \epsilon_{b}=\varepsilon^{1}\right\}$.

- Find the position of the shock-waves at time $\varepsilon^{1}, h_{a}=L_{a}+w_{a} \varepsilon^{1}$.

- Find the new length of the parts for the time $\varepsilon^{1}$,

$$
\begin{array}{lr}
l_{a, 1}^{1}=L_{a}+w_{a, 1} \varepsilon^{1} & \forall a \in \mathcal{Q} \\
l_{a, 2}^{1}=-w_{a, 1} \varepsilon^{1} & \forall a \in \mathcal{Q} \\
l_{a, 1}^{1}=L_{a} & \forall a \in \mathcal{A} \backslash \mathcal{Q} .
\end{array}
$$

- Find the number of vehicles that have passed through the network until the time $\varepsilon^{0}$. The method is based on a first order traffic flow model the transitions between the different states of a link is linear. Thus the number of vehicles traversed the link between two time steps $\varepsilon^{n}$ and $\varepsilon^{n+1}$, can be found as

$$
\sum_{i \in \mathcal{P}_{a}}\left(Q_{a, i} \frac{l_{a, i}^{n}+l_{a, i}^{n+1}}{2 L_{a}}\left(\varepsilon^{n+1}-\varepsilon^{n}\right)\right) \quad \forall a \in \mathcal{A}
$$

Therefore, for $n=0$,

$$
N=\sum_{i \in \mathcal{P}_{a}}\left(Q_{a, i} \frac{l_{a, i}^{0}+l_{a, i}^{1}}{2 L_{a}} \varepsilon^{1}\right) .
$$

- Find the $g$ of pollutant emitted until time $e^{1}$. Accordingly, the $g$ of pollutant emitted during one time step, can be found as

$$
\sum_{i \in \mathcal{P}_{a}}\left(e\left(V_{a, i}\right) Q_{a, i} \frac{l_{a, i}^{n}+l_{a, i}^{n+1}}{2}\left(\varepsilon^{n+1}-\varepsilon^{n}\right)\right),
$$

for each pollutant $p$ and $a \in \mathcal{A}$, where $e_{a}^{p}(v)$ is the emission factor in $\mathrm{g} /$ vehkm as a function of average speed $v$.

For $n=0$, 


$$
E_{a}^{p}=\sum_{i \in \mathcal{P}_{a}}\left(e_{a}^{p}\left(V_{a, i}\right) Q_{a, i} \frac{l_{a, i}^{0}+l_{a, i}^{1}}{2} \varepsilon^{1}\right)
$$

- Determine an event type indicator $d$ and set $d=1$.

Note: Since the demand is constant (second static assumption), there is not any change regarding the conditions at the upstream end of the links. Hence, no forwards moving shock-wave is created. With only backward moving shock-waves, only two types of events are possible: the first type of event occurs when a backward moving shock-wave hits the upstream end of the link, while the second type of event when a backward moving shockwave meets a slower backward moving shock-wave. We add a third type of event when the number of vehicles that has passed by a link is equal to the demand $x_{a}$ of the link. Since we assume instant loading (first static assumption), we also assume instant unloading of a link.

\section{Main loop}

1 Set $n:=n+1$.

2 If $d=1$ (a backward moving shock-wave hits the upstream end of the link $b$ )

- Update for the event link $b$ the number of waves $W_{b}:=$ $W_{b}-1$ and the number of parts $P_{b}:=P_{b}-1$, as well as the sets $\mathcal{W}_{b}=\left\{1,2, \ldots, W_{b}\right\}, \mathcal{P}_{b}=\left\{1,2, \ldots, P_{b}\right\}$.

- Find the increase in the capacity of link $b: \zeta=Q_{b, 2}-Q_{b, 1}$.

- Update for the event link $b$ the flow, the density, the speed and the length of the parts

$$
\begin{aligned}
Q_{b, i} & =Q_{b, i+1} \quad \forall i \in \mathcal{P}_{b} \\
K_{b, i} & =K_{b, i+1} \quad \forall i \in \mathcal{P}_{b} \\
V_{b, i} & =Q_{b, i} / K_{b, i} \quad \forall i \in \mathcal{P}_{b} \\
l_{b, i}^{n} & =l_{b, i+1}^{n} \quad \forall i \in \mathcal{P}_{b} .
\end{aligned}
$$

- Node model 
(a) Determine a set with all the routes route that include the link $b, \tilde{\mathcal{C}}=\left\{k \in \mathcal{K} \mid \delta_{b, k}^{r s}=1\right\}$. For each route $k \in \tilde{\mathcal{C}}$, find the position $\eta$ of link $b$ such as $\mathcal{D}_{\eta}^{r s, k}=$ $b$. Determine the set of links $\tilde{\mathcal{B}}$, including the links upstream link $b, \tilde{\mathcal{B}}=\left\{a \in \mathcal{A} \mid a=\mathcal{D}_{\eta-1}^{r s, k}, k \in \tilde{\mathcal{C}}\right\}$.

(b) At the time $\varepsilon^{n}$, when the backwards moving shockwave hits the upstream end of link $b$, another backwards moving shock-wave is created at the most downstream end of the links upstream the $b$ because of the spill-back.

Set

$$
\begin{aligned}
W_{a} & :=W_{a}+1 & & \forall a \in \tilde{\mathcal{B}} \\
P_{a} & :=P_{a}+1 & & \forall a \in \tilde{\mathcal{B}} \\
\mathcal{W}_{b} & =\left\{1,2, \ldots, W_{b}\right\} & & \forall a \in \tilde{\mathcal{B}} \\
\mathcal{P}_{b} & =\left\{1,2, \ldots, P_{b}\right\} & & \forall a \in \tilde{\mathcal{B}}
\end{aligned}
$$

(c) Restrict proportionally the outflows of the links upstream the link $b$. The node inflow capacity is increased by $\zeta$. Therefore, for each link $a \in \tilde{\mathcal{B}}$ upstream the link $b$, the outflow is restricted proportionally

$$
\begin{aligned}
& Q_{a, P_{a}}= \\
& Q_{a, P_{a}}+\zeta \frac{Q_{a, P_{a}-1}}{\sum_{j \in \tilde{\mathcal{B}}} Q_{j, P_{j}}} .
\end{aligned}
$$

(d) Set the position of new shock-waves $p_{a, W_{a}}^{n}=L_{a}$, and update the densities the speeds and the length of the newly created parts for the links $a \in \tilde{\mathcal{B}}$

$$
\begin{aligned}
K_{a, P_{a}} & =k_{a}\left(Q_{a, P_{a}} \mid \text { congested }\right) & & \forall a \in \tilde{\mathcal{B}} \\
V_{a, P_{a}} & =Q_{a, P_{a}} / K_{a, P_{a}} & & \forall a \in \tilde{\mathcal{B}} \\
l_{a, P_{a}}^{n} & =0 & & \forall a \in \tilde{\mathcal{B}}
\end{aligned}
$$

End of node model

If $d=2$ (a backward moving shock-wave meets the slower $\nu^{\text {th }}$ backward moving shock-wave in link $b$ ) 
- Update for the event link $b$ the number of waves $W_{b}:=$ $W_{b}-1$ and the number of parts $P_{b}:=P_{b}-1$, as well as the sets $\mathcal{W}_{b}=\left\{1,2, \ldots, W_{b}\right\}, \mathcal{P}_{b}=\left\{1,2, \ldots, P_{b}\right\}$.

- Update for the event link $b$ the flow, the density, the speed and the length of the parts

$$
\begin{array}{rrr}
Q_{b, i}:=Q_{b, i}, K_{b, i}:=K_{b, i} & \forall i \in\left\{j \in \mathcal{P}_{b} \mid j<=\nu\right\} \\
Q_{b, i}=Q_{b, i+1}, K_{b, i}=K_{b, i+1}, l_{b, i}^{n}=l_{b, i+1}^{n} & \forall i \in\left\{j \in \mathcal{P}_{b} \mid j>\nu\right\} \\
V_{b, i}=Q_{b, i} / K_{b, i} & \forall i \in \mathcal{P}_{b} .
\end{array}
$$

- Update for the event link $b$ the position of the shock-waves

$$
\begin{array}{rlrl}
h_{b, i} & =h_{b, i} & \forall i \in\left\{j \in \mathcal{W}_{b} \mid j<=\nu\right\} \\
h_{b, i}=h_{b, i+1} & \forall i \in\left\{j \in \mathcal{W}_{b} \mid j>\nu\right\}
\end{array}
$$

If $d=3$ (the flow that has passed by a link $b$ is equal to the demand $x_{b}$ of the link.)

- Update for the event link $b$ the number of waves $W_{b}=0$ and the number of parts $P_{b}=1$, as well as the sets $\mathcal{W}_{b}=\emptyset$, $\mathcal{P}_{b}=\{1\}$.

- Find the increase in the capacity of link $b: \zeta=Q_{b, 1}$.

- Update for the event link $b$ the flow, the density, the speed and the length of the parts

$$
\begin{aligned}
Q_{b, i} & =0 & & \forall i \in \mathcal{P}_{b} \\
K_{b, i} & =0 & & \forall i \in \mathcal{P}_{b} \\
V_{b, i} & =0 & & \forall i \in \mathcal{P}_{b} \\
l_{b, i}^{n} & =L_{b} & & \forall i \in \mathcal{P}_{b} .
\end{aligned}
$$

- Node model

(a) Same as the node model for $d=1$.

(b) Same as the node model for $d=1$. 
(c) Increase proportionally the outflows of the links upstream the link $b$. The node inflow capacity is increased by $\zeta$. For each $a \in \tilde{\mathcal{B}}$

$$
\begin{aligned}
& Q_{a, P_{a}}= \\
& \min \left\{x_{a}, Q_{a, P_{a}-1}+\zeta \frac{Q_{a, P_{a}}}{\sum_{j \in \tilde{\mathcal{B}}_{n}} Q_{j, P_{j}}}\right\} .
\end{aligned}
$$

(d) Set the position of new shock-waves $h_{a, W_{a}}=L_{a}$, and update the densities the speeds and the length of the newly created parts

$$
\begin{aligned}
K_{a, P_{a}} & =k_{a}\left(Q_{a, P_{a}} \mid \text { uncongested }\right) & & \forall a \in \tilde{\mathcal{B}} \\
V_{a, P_{a}} & =Q_{a, P_{a}} / K_{a, P_{a}} & & \forall a \in \tilde{\mathcal{B}} \\
l_{a, P_{a}}^{n} & =0 & & \forall a \in \tilde{\mathcal{B}} .
\end{aligned}
$$

End of node model

3 Update the set $\mathcal{Q}, \mathcal{Q}=\left\{a \in \mathcal{A} \mid W_{a}>0\right\}$.

4 Update the shock-waves speeds

$$
w_{a, i}=\frac{Q_{a, i+1}-Q_{a, i}}{K_{a, i+1}-K_{a, i}} \quad \forall i \in W_{a} \forall a \in \mathcal{Q}
$$

5 Find the next event.

- Event type 1

For each link $a \in \mathcal{Q}$ find the candidate times for the next event $\tilde{\epsilon}_{a}=l_{a, 1}^{n} / w_{a, 1}$. Then find the the shortest candidate time for the event type $1, \epsilon_{1}=\min _{a \in \mathcal{Q}} \tilde{\epsilon}_{a}$, and the associated with that time link, $\tilde{b}_{1}$ such as $\tilde{\epsilon}_{\tilde{b}_{1}}=\epsilon_{1}$.

- Event type 2

Determine a set which includes the links with more than one wave, $\tilde{\mathcal{Q}}=\left\{a \in \mathcal{Q} \mid W_{a}>1\right\}$. Then for each link $a \in \tilde{\mathcal{Q}}$ find

$$
\epsilon_{a, i}^{*}=\frac{h_{a, j+1}-h_{a, j}}{w_{a, j}-w_{a, j+1}} \forall i \in \mathcal{P}_{a}
$$


that denotes the time that the shock-wave $i+1$ needs to reach the shock-wave $i$. Therefore, the candidate event times can be found as $\tilde{\epsilon}_{a}=\min _{i \in \mathcal{P}_{a}}\left\{\epsilon_{a, i}^{*} \mid \epsilon_{a, i}^{*}>0\right\}$. Then, find the shortest candidate time for event type $2 \epsilon_{2}=$ $\min _{a \in \tilde{\mathcal{Q}}} \tilde{\epsilon}_{a}$ and the associated with that time link, $\tilde{b}_{2}$ such as $\tilde{\epsilon}_{\tilde{b}_{2}}=\epsilon_{2}$. Set $\nu=\operatorname{argmin}_{i \in \mathcal{P}_{\tilde{b}_{2}}}\left\{\epsilon_{\tilde{b}_{2}, i}^{*} \mid \epsilon_{\tilde{b}_{2}, i}^{*}>0\right\}$.

- Event type 3

If $\varepsilon^{n}<1$, then $\epsilon_{3}=\infty$. Else, for each link $a \in \mathcal{A}$, find the time $\tilde{\epsilon}_{a}$ when the number of vehicles have traversed the link equals to the demand $x_{a}$, such as

$$
\begin{aligned}
N_{a} & +Q_{a, 1} \frac{2 l_{a, 1}^{n}+w_{a, 1} \tilde{\epsilon}_{a}}{2 L_{a}}\left(\tilde{\epsilon}_{a}-\varepsilon^{n}\right) \\
& +\sum_{i \in\left\{2, \ldots, W_{a}-1\right\}}\left(Q_{a, i} \frac{2 l_{a, i}^{n}+w_{a, i-1} \tilde{\epsilon}_{a}-w_{a, i} \tilde{\epsilon}_{a}}{2 L_{a}}\left(\tilde{\epsilon}_{a}-\varepsilon^{n}\right)\right) \\
& +Q_{a, P_{a}} \frac{2 l_{a, P_{a}}^{n}-w_{a, W_{a}} \tilde{\epsilon}_{a}}{2 L_{a}}\left(\tilde{\epsilon}_{a}-\varepsilon^{n}\right)=x_{a} \quad \forall a \in \mathcal{Q}
\end{aligned}
$$

Solving the above equation for $\tilde{\epsilon}_{a}$, we obtain a set of solutions

$$
\mathcal{S}_{a}=\left\{\frac{-\beta+\sqrt{\beta^{2}-4 \alpha \gamma}}{2 \alpha}, \frac{-\beta-\sqrt{\beta^{2}-4 \alpha \gamma}}{2 \alpha}\right\} \quad \forall a \in \mathcal{Q}
$$

where

$$
\begin{aligned}
\alpha= & Q_{a, 1} w_{a, 1}+\sum_{i \in\left\{2, \ldots, W_{a}-1\right\}}\left(Q_{a, i} w_{a, i-1}\right) \\
& -\sum_{i \in\left\{2, \ldots, W_{a}-1\right\}}\left(Q_{a, i} w_{a, i}\right)-Q_{a, P_{a}} w_{a, W_{a}}, \\
\beta= & Q_{a, 1} 2 L_{a, 1}^{n}+\sum_{i \in\left\{2, \ldots, W_{a}-1\right\}}\left(Q_{a, i} 2 l_{a, i}^{n}\right)+Q_{a, P_{a}} 2 l_{a, P_{a}}^{n} \\
& -\varepsilon^{n}\left(Q_{a, 1} w_{a, 1}+\sum_{i \in\left\{2, \ldots, W_{a}-1\right\}}\left(Q_{a, i} w_{a, i-1}\right)\right) \\
& +\varepsilon^{n}\left(\sum_{i \in\left\{2, \ldots, W_{a}-1\right\}}\left(Q_{a, i} w_{a, i}\right)+Q_{a, P_{a}} w_{a, W_{a}}\right)
\end{aligned}
$$




$$
\begin{aligned}
\gamma= & -\varepsilon^{n}\left(Q_{a, 1} 2 L_{a, 1}+\sum_{i \in\left\{2, \ldots, W_{a}-1\right\}}\left(Q_{a, i} 2 l_{a, i}^{n}\right)+Q_{a, P_{a}} 2 l_{a, P_{a}}^{n}\right) \\
& -2 L_{a}\left(x_{a}-N_{a}\right)
\end{aligned}
$$

Set

$$
\tilde{\epsilon}_{a}=\min \left\{j \in \mathcal{S}_{a} \mid j-\varepsilon^{n}>0\right\} \quad \forall a \in \mathcal{Q}
$$

and

$$
\tilde{\epsilon}_{a}=\frac{x_{a}-N_{a}+\varepsilon^{n} Q_{a, 1}}{Q_{a, 1}} \quad \forall a \in \mathcal{A} \backslash \mathcal{Q} .
$$

Then, find the shortest candidate time for event type 3 $\epsilon_{3}=\min _{a \in \mathcal{Q}} \tilde{\epsilon}_{a}$ and the associated with that time link, $\tilde{b}_{3}$ such as $\tilde{\epsilon}_{\tilde{b}_{3}}=\epsilon_{3}$.

- Set the next event time $\varepsilon^{n+1}=\varepsilon^{n}+\min _{i=1,2,3}\left\{\epsilon_{i}\right\}$. Update the type of the next event, $d=\arg \min _{i=1,2,3}\left\{\epsilon_{i}\right\}$ and the new event link, $b=\tilde{b}_{d}$.

6 Update the position of shock-waves

$$
h_{a, j}:=h_{a, j}+w_{a, 1}\left(\varepsilon^{n+1}-\varepsilon^{n}\right) \quad \forall j \in \mathcal{W}_{a}, \forall a \in \mathcal{Q} .
$$

7 Update the lengths of the parts

$$
\begin{aligned}
l_{a, 1}^{n+1}= & l_{a, 1}^{n}+w_{a, 1}\left(\varepsilon^{n+1}-\varepsilon^{n}\right) \quad \forall a \in \mathcal{Q} \\
l_{a, i}^{n+1}= & l_{a, i}^{n}-w_{a, i-1}\left(\varepsilon^{n+1}-\varepsilon^{n}\right) \\
& +w_{a, i}\left(\varepsilon^{n+1}-\varepsilon^{n}\right) \quad \forall i \in\left\{2, \ldots, P_{a}-1\right\}, \forall a \in \mathcal{Q} \\
l_{a, P_{a}}^{n+1}= & l_{a, P_{a}}^{n}-w_{a, P_{a}}\left(\varepsilon^{n+1}-\varepsilon^{n}\right) \quad \forall a \in \mathcal{Q} \\
l_{a, 1}^{n+1}= & L_{a} \quad \forall a \in \mathcal{A} \backslash \mathcal{Q} .
\end{aligned}
$$

8 Update the number of vehicles have passed by until $\varepsilon^{n+1}$,

$$
N_{a}:=N_{a}+\sum_{i \in \mathcal{P}_{a}}\left(Q_{a, i} \frac{l_{a, i}^{n}+l_{a, i}^{n+1}}{2 L_{a}}\left(\varepsilon^{n+1}-\varepsilon^{n}\right)\right) \quad \forall a \in \mathcal{A} .
$$


9 Update the $q$ of pollutant emitted until $\varepsilon^{n+1}$,

$$
E_{a}^{p}:=E_{a}^{p}+\sum_{i \in \mathcal{P}_{a}}\left(e_{a}^{p}\left(V_{a, i}\right) Q_{a, i} \frac{l_{a, i}^{n}+l_{a, i}^{n+1}}{2}\left(\varepsilon^{n+1}-\varepsilon^{n}\right)\right)
$$

10 If $\sum_{a \in \mathcal{A}} N_{a}=\sum_{a \in \mathcal{A}} x_{a}$ stop, else return to step 1.

End 



\section{Bibliography}

Aguilera, A. and Lebacque, M. (2010). "Dynamic estimation of traffic emissions in metropolitan road networks". In: Proceedings of the 12th WCTR, Lisbon, Portugal.

Ahmed, K. I. (1999). "Modeling drivers' acceleration and lane changing behavior". eng. Thesis. Massachusetts Institute of Technology.

Akcelik, R. (1991a). "Travel time functions for transport planning purposes: Davidson's function, its time dependent form and alternative travel time function". In: Australian Road Research 21.3.

Akcelik, R. (1991b). "Travel time functions for transport planning purposes: Davidson's function, its time dependent form and alternative travel time function". In: Australian Road Research 21.3.

Allström, A., Archer, J., Bayen, A., Blandin, S., Butler, J., Gundlegård, D., Koutsopoulos, H, Lundgren, J., Rahmani, M., and Tossavainen, O.-P. (2011). "Mobile Millennium Stockholm". In: 2nd International Conference on Models and Technologies for Intelligent Transportation Systems. Leuven, Belgium.

Allström, A., Barceló, J., Ekström, J., Grumert, E., Gundlegård, D., and Rydergren, C. (2017). "Traffic management for smart cities". In: Designing, Developing, and Facilitating Smart Cities. Springer, pp. 211-240.

Bai, S., Chiu, Y.-C. E., and Niemeier, D. A. (2007a). "A comparative analysis of using trip-based versus link-based traffic data for regional mobile source emissions estimation". In: Atmospheric Environment 41.35, pp. 7512-7523.

Bai, S., Nie, Y., and Niemeier, D. A. (2007b). "The impact of speed post-processing methods on regional mobile emissions estimation". 
In: Transportation Research Part D: Transport and Environment 12.5 , pp. 307-324.

Basarić, V., Đorić, V., Bogdanović, V., Mitrović, J., and Jović, J. (2014). "Effects of Traffic on NO 2 and PM 10 Emissions in Novi Sad." In: Polish Journal of Environmental Studies 23.5.

Batterman, S., Chambliss, S., and Isakov, V. (2014). "Spatial resolution requirements for traffic-related air pollutant exposure evaluations". In: Atmospheric environment 94, pp. 518-528.

Batterman, S., Cook, R., and Justin, T. (2015). "Temporal variation of traffic on highways and the development of accurate temporal allocation factors for air pollution analyses". In: Atmospheric environment 107, pp. 351-363.

Beckmann, M., McGuire, C. B., and Winsten, C. B. (1956). Studies in the Economics of Transportation. Tech. rep.

Beevers, S. D. and Carslaw, D. C. (2005). "The impact of congestion charging on vehicle emissions in London". In: Atmospheric Environment 39.1, pp. 1-5.

Bezdek, J. C., Ehrlich, R., and Full, W. (1984). "FCM: The fuzzy cmeans clustering algorithm". In: Computers $\& 3$ Geosciences 10.2-3, pp. 191-203.

Björketun, U and Carlsson, A (2005). Trafikvariation över dygnet. Tech. rep. VTI.

Bliemer, M., Brederode, L., Wismans, L., and Smits, E. S. (2012). "Quasi-dynamic traffic assignment: static traffic assignment with queuing and spillback". In: Proceedings of the 94th Annual Meeting of the Trasportation Research Board, Washington, D.C., USA.

Borge, R., Miguel, I. de, Paz, D. de la, Lumbreras, J., Pérez, J., and Rodríguez, E. (2012). "Comparison of road traffic emission models in Madrid (Spain)". In: Atmospheric Environment 62, pp. 461471.

Brederode, L., Bliemer, M., and Wismans, L. (2010). "STAQ: Static Traffic Assignment with Queing". In: European Transport Conference. 
Brederode, L., Pel, A., Wismans, L., Romph, E. de, and Hoogendoorn, S. (2018). "Static Traffic Assignment with Queuing: model properties and applications". In: Transportmetrica A: Transport Science, pp. 1-36.

Briggs, D. (2005). "The role of GIS: coping with space (and time) in air pollution exposure assessment". In: Journal of Toxicology and Environmental Health, Part A 68.13-14, pp. 1243-1261.

Bundschuh, M., Vortisch, P., PTV AG, K., Van Vuuren, T., and Mott McDonald, U. (2006a). "Modelling queues in static traffic assignment". In: Proceedings of the European Transport Conference, p. 2006.

Bundschuh, M., Vortisch, P., Van Vuuren, T., and Mott McDonald, U. K. (2006b). "Modelling queues in static traffic assignment". In: Proceedings of the European Transport Conference, p. 2006.

Burghout, W., Koutsopoulos, H. N., and Andreasson, I. (2006). "A discrete-event mesoscopic traffic simulation model for hybrid traffic simulation". In: Intelligent Transportation Systems Conference, 2006. ITSC'06. IEEE. IEEE, pp. 1102-1107.

Caliński, T. and Harabasz, J. (1974). "A dendrite method for cluster analysis". In: Communications in Statistics-theory and Methods 3.1 , pp. $1-27$.

Chandler, R. E., Herman, R., and Montroll, E. W. (1958). "Traffic Dynamics: Studies in Car Following”. In: Operations Research 6.2, pp. $165-184$.

Chiu, Y.-C., Bottom, J., Mahut, M., Paz, A., Balakrishna, R., Waller, T., and Hicks, J. (2011). "Dynamic traffic assignment: A primer". In: Transportation Research E-Circular E-C153.

Coelho, M. C., Fontes, T., Bandeira, J. M., Pereira, S. R., Tchepel, O., Dias, D., Sá, E., Amorim, J. H., and Borrego, C. (2014). "Assessment of potential improvements on regional air quality modelling related with implementation of a detailed methodology for traffic emission estimation". In: Science of the Total Environment 470, pp. $127-137$.

Daganzo, C. F. (1994). "The cell transmission model: A dynamic representation of highway traffic consistent with the hydrodynamic 
theory". In: Transportation Research Part B: Methodological 28.4, pp. 269-287.

Daganzo, C. F. (1995). "The cell transmission model, part II: Network traffic". In: Transportation Research Part B: Methodological 29.2, pp. 79-93.

Davidson, K. (1966). "A flow travel time relationship for use in transportation planning". In: Australian Road Research Board (ARRB) Conference, 3rd, 1966, Sydney. Vol. 3. 1.

Dowling, R. and Skabardonis, A. (1992). "Improving average travel speeds estimated by planning models". In: Transportation Research Record 1366.

Duan, N. (1982). "Models for human exposure to air pollution". In: Environment International 8.1-6, pp. 305-309.

EEA (2014). Air Quality in Europe - 2014. Tech. rep. 5/2014. ISSN 1977-8449. European Environment Agency.

EEA (2016a). Air Quality in Europe - 2016. Tech. rep. 28/201. ISBN 978-92-9213-847-9. European Environment Agency.

EEA (2016b). EMEP/EEA air pollutant emission inventory guidebook 2016. Tech. rep. ECE/EB.AIR/125. ISSN 1977-8449. European Environment Agency.

EEA (2017). European Union emission inventory report 1990-2015 under the UNECE Convention on Long-range Transboundary Air Pollution (LRTAP). Tech. rep. 9/2017. ISSN 1977-8449. European Environment Agency.

Eliasson, J. (2009). "A cost-benefit analysis of the Stockholm congestion charging system". In: Transportation Research Part A: Policy and Practice 43.4, pp. 468-480.

Ferm, M. and Sjöberg, K. (2015). "Concentrations and emission factors for PM 2.5 and PM 10 from road traffic in Sweden". In: Atmospheric Environment 119, pp. 211-219.

Franco, V., Fontaras, G., and Dilara, P. (2012). "Towards Improved Vehicle Emissions Estimation in Europe". In: Procedia - Social and Behavioral Sciences. Transport Research Arena 2012 48, pp. 13041313. 
Franco, V., Kousoulidou, M., Muntean, M., Ntziachristos, L., Hausberger, S., and Dilara, P. (2013). "Road vehicle emission factors development: A review". In: Atmospheric Environment 70, pp. 8497 .

Frank, M. and Wolfe, P. (1956). "An algorithm for quadratic programming". In: Naval Research Logistics (NRL) 3.1-2, pp. 95110.

Friesz, T. L., Luque, J., Tobin, R. L., and Wie, B.-W. (1989). "Dynamic network traffic assignment considered as a continuous time optimal control problem". In: Operations Research 37.6, pp. 893901.

Fritzsche, H. T. (1994). "A model for traffic simulation". In:

Fu, M., Kelly, J. A., and Clinch, J. P. (2017). "Estimating annual average daily traffic and transport emissions for a national road network: A bottom-up methodology for both nationally-aggregated and spatially-disaggregated results". In: Journal of Transport Geography 58, pp. 186-195.

Gastaldi, M., Rossi, R., Gecchele, G., and Della Lucia, L. (2013). "Annual average daily traffic estimation from seasonal traffic counts". In: Procedia-Social and Behavioral Sciences 87, pp. 279-291.

Gately, C. K., Hutyra, L. R., Peterson, S., and Wing, I. S. (2017). "Urban emissions hotspots: Quantifying vehicle congestion and air pollution using mobile phone GPS data". In: Environmental Pollution 229, pp. 496-504.

Gazis, D. C., Herman, R., and Rothery, R. W. (1961). "Nonlinear Follow-the-Leader Models of Traffic Flow". In: Operations Research 9.4 , pp. 545-567.

Gentile, G. (2015). "Using the general link transmission model in a dynamic traffic assignment to simulate congestion on urban networks". In: Transportation Research Procedia 5, pp. 66-81.

Gilbert, N. L., Woodhouse, S., Stieb, D. M., and Brook, J. R. (2003). "Ambient nitrogen dioxide and distance from a major highway". In: Science of the Total Environment 312.1-3, pp. 43-46. 
Gipps, P. G. (1981). "A Behavioural Car-Following Model for Computer Simulation". In: Transportation Research Part B: Methodological 15.2, pp. 105-111.

Gipps, P. G. (1986). "A model for the structure of lane-changing decision". In: Transportation Research Part B: Methodological 20.5, pp. 403-414.

Greenshields, B. D. (1934). "A study of traffic capacity". In: A Study of Traffic Capacity 14.

Gulliver, J. and Briggs, D. (2011). "STEMS-Air: A simple GIS-based air pollution dispersion model for city-wide exposure assessment". In: Science of the total environment 409.12, pp. 2419-2429.

Ha, J.-A. and Oh, J.-S. (2014). "Estimating Annual Average Daily traffic using Daily Adjustment Factor". In: Journal of emerging trends in Computing and Information Sciences, p. 580.

Haan, P. de (1999). "On the use of density kernels for concentration estimations within particle and puff dispersion models". In: Atmospheric Environment 33.13, pp. 2007-2021.

Hartigan, J. A. and Wong, M. A. (1979). "Algorithm AS 136: A kmeans clustering algorithm". In: Journal of the Royal Statistical Society. Series C (Applied Statistics) 28.1, pp. 100-108.

Holmes, N. S. and Morawska, L. (2006). "A review of dispersion modelling and its application to the dispersion of particles: an overview of different dispersion models available". In: Atmospheric environment 40.30, pp. 5902-5928.

Hoogendoorn Serge P, B. P. H. (2001). "State-of-the-art of vehicular traffic flow modelling". In: Proceedings of the Institution of Mechanical Engineers, Part I: Journal of Systems and Control Engineering 215.4, pp. 283-303.

Janson, B. N. (1991). "Dynamic traffic assignment for urban road networks". In: Transportation Research Part B: Methodological 25.23 , pp. $143-161$.

Jeng, S.-T., Nesamani, K., and Ritchie, S. G. (2013). "A new approach to estimate vehicle emissions using inductive loop detector data". In: Journal of Intelligent Transportation Systems 17.3, pp. 179190. 
Jerrett, M., Arain, A., Kanaroglou, P., Beckerman, B., Potoglou, D., Sahsuvaroglu, T., Morrison, J., and Giovis, C. (2005). "A review and evaluation of intraurban air pollution exposure models". In: Journal of Exposure Science and Environmental Epidemiology 15.2, p. 185.

Jing, B., Wu, L., Mao, H., Gong, S., He, J., Zou, C., Song, G., Li, X., and $\mathrm{Wu}, \mathrm{Z}$. (2016). "Development of a vehicle emission inventory with high temporal-spatial resolution based on NRT traffic data and its impact on air pollution in Beijing-Part 1: Development and evaluation of vehicle emission inventory". In: Atmospheric Chemistry and Physics 16.5, pp. 3161-3170.

Johansson, C., Burman, L., and Forsberg, B. (2009). "The effects of congestions tax on air quality and health". In: Atmospheric Environment 43.31, pp. 4843-4854.

Keller, M (2010). Handbook of emission factors for road transport (HBEFA) 3.1. Tech. rep. Quick reference. Tech. rep., INFRAS. http://www. hbefa. net.

Keller, M and Kljun, N (2007). "ARTEMIS Road Emission ModelModel Description". In: EU project ARTEMIS deliverable13, INFRAS Report, pp. 1-199.

Kholod, N, Evans, M, Gusev, E, Yu, S, Malyshev, V, Tretyakova, S, and Barinov, A (2016). "A methodology for calculating transport emissions in cities with limited traffic data: Case study of diesel particulates and black carbon emissions in Murmansk". In: Science of the Total Environment 547, pp. 305-313.

Larsson, T. and Patriksson, M. (1992). "Simplicial decomposition with disaggregated representation for the traffic assignment problem". In: Transportation Science 26.1, pp. 4-17.

Larsson, T. and Patriksson, M. (1995). "An augmented Lagrangean dual algorithm for link capacity side constrained traffic assignment problems". In: Transportation Research Part B: Methodological 29.6, pp. $433-455$.

Leeuwen, A. (2011). "Static traffic assignment with queuing". MA thesis. University of Twente. 
Leutzbach, W. (1988). Introduction to the theory of traffic flow. Vol. 47. Springer.

Lighthill, M. J. and Whitham, G. B. (1955). "On Kinematic Waves. II. A Theory of Traffic Flow on Long Crowded Roads". en. In: Proceedings of the Royal Society of London A: Mathematical, Physical and Engineering Sciences 229.1178, pp. 317-345.

Lindhjem, C. E., Pollack, A. K., DenBleyker, A., and Shaw, S. L. (2012). "Effects of improved spatial and temporal modeling of on-road vehicle emissions". In: Journal of the Air 86 Waste Management Association 62.4, pp. 471-484.

Losses, R. (1994). "Automobile Emissions: An Overview". In:

Maerivoet, S. and De Moor, B. (2005). "Transportation Planning and Traffic Flow Models". In: arXiv:physics/0507127. arXiv: physics/0507127.

Mcnally, M. G. (2000). "The four step model". In: Handbook of Transport Modelling. Oxford, Pergamon Press, pp. 35-52.

Merchant, D. K. and Nemhauser, G. L. (1978). "A model and an algorithm for the dynamic traffic assignment problems". In: Transportation science 12.3, pp. 183-199.

Monn, C. (2001). "Exposure assessment of air pollutants: a review on spatial heterogeneity and indoor/outdoor/personal exposure to suspended particulate matter, nitrogen dioxide and ozone". In: Atmospheric environment 35.1, pp. 1-32.

Muller-Perriand, K. (2014). "Effects of temporal traffic data aggregation level on the estimation of air pollutant emissions using a COPERT 4 based model: application to A7 motorway (France)". In: Proceedings of the 5th Transport Research Arena, Paris, France.

Namdeo, A., Mitchell, G., and Dixon, R. (2002). "TEMMS: an integrated package for modelling and mapping urban traffic emissions and air quality". In: Environmental Modelling $\mathcal{E}$ Software 17.2, pp. $177-188$.

Nathan Gartner C. J. Messer, A. K. R. (2001). Traffic flow theory: A state-of-the-art report. Tech. rep.

Negrenti, E. (1999). "The 'corrected average speed'approach in ENEA's TEE model: an innovative solution for the evaluation of the ener- 
getic and environmental impacts of urban transport policies". In: Science of the total environment 235.1, pp. 411-413.

Nesamani, K. S., Chu, L., McNally, M. G., and Jayakrishnan, R. (2007). "Estimation of vehicular emissions by capturing traffic variations". In: Atmospheric Environment 41.14, pp. 2996-3008.

Neudorff, L. G., Randall, J. E., Reiss, R., and Gordon, R. (2003). Freeway management and operations handbook. Tech. rep.

Newell, G. F. (1993). "A simplified theory of kinematic waves in highway traffic, part II: Queueing at freeway bottlenecks". In: Transportation Research Part B: Methodological 27.4, pp. 289-303.

Nicholson, S. E. (1975). "A pollution model for street-level air". In: Atmospheric Environment (1967) 9.1, pp. 19-31.

Nie, X. and Zhang, H. M. (2005). "A comparative study of some macroscopic link models used in dynamic traffic assignment". In: Networks and Spatial Economics 5.1, pp. 89-115.

Nie, Y., Zhang, H., and Lee, D.-H. (2004). "Models and algorithms for the traffic assignment problem with link capacity constraints". In: Transportation Research Part B: Methodological 38.4, pp. 285312 .

Ntziachristos, L., Gkatzoflias, D., Kouridis, C., and Samaras, Z. (2009). "COPERT: a European road transport emission inventory model". In: Information technologies in environmental engineering, pp. 491504 .

Nyhan, M., Sobolevsky, S., Kang, C., Robinson, P., Corti, A., Szell, M., Streets, D., Lu, Z., Britter, R., Barrett, S. R., et al. (2016). "Predicting vehicular emissions in high spatial resolution using pervasively measured transportation data and microscopic emissions model". In: Atmospheric Environment 140, pp. 352-363.

Oke, T. R. (1988). "Street design and urban canopy layer climate". In: Energy and buildings 11.1, pp. 103-113.

Ott, W. R. (1982). "Concepts of human exposure to air pollution". In: Environment International 7.3, pp. 179-196.

Pastorello, C and Melios, G (2016). "Explaining road transport emissions: a non-technical guide". In: 
Patriksson, M. (2015). The traffic assignment problem: models and methods. Courier Dover Publications.

Peeta, S. and Ziliaskopoulos, A. K. (2001). "Foundations of dynamic traffic assignment: The past, the present and the future". In: Networks and spatial economics 1.3-4, pp. 233-265.

Peters, G. P. (2008). "From production-based to consumption-based national emission inventories". In: Ecological economics 65.1, pp. 1323.

Pipes, L. A. (1953). "An Operational Analysis of Traffic Dynamics". In: Journal of Applied Physics 24, pp. 274-281.

Protocol, K. (1997). "United Nations framework convention on climate change". In: Kyoto Protocol, Kyoto 19.

Raadsen, M. P., Bliemer, M. C., and Bell, M. G. (2016). "An efficient and exact event-based algorithm for solving simplified first order dynamic network loading problems in continuous time". In: Transportation Research Part B: Methodological 92, pp. 191-210.

Richards, P. I. (1956). "Shock Waves on the Highway". In: Operations Research 4.1, pp. 42-51.

Ryu, B. Y., Jung, H. J., and Bae, S. H. (2015). "Development of a corrected average speed model for calculating carbon dioxide emissions per link unit on urban roads". In: Transportation Research Part D: Transport and Environment 34, pp. 245-254.

Seo, T., Bayen, A. M., Kusakabe, T., and Asakura, Y. (2017). "Traffic state estimation on highway: A comprehensive survey". In: Annual Reviews in Control 43, pp. 128-151.

Sheffi, Y. (1985). Urban transportation networks.

Silverman, B. W. (1986). "Density estimation for statistics and data analysis". In: Monographs on Statistics and Applied Probability 26.

Skabardonis, A. and Dowling, R. (1997). "Improved speed-flow relationships for planning applications". In: Transportation Research Record: Journal of the Transportation Research Board 1572, pp. 1823. 
Smit, R., Poelman, M., and Schrijver, J. (2008). "Improved road traffic emission inventories by adding mean speed distributions". In: Atmospheric Environment 42.5, pp. 916-926.

Smit, R., Dia, H., and Morawska, L. (2009). "Road traffic emission and fuel consumption modelling: trends, new developments and future challenges". In: Traffic Related Air Pollution and Internal Combustion Engines. Nova Publishers USA.

Smit, R., Ntziachristos, L., and Boulter, P. (2010). "Validation of road vehicle and traffic emission models-A review and meta-analysis". In: Atmospheric environment 44.25, pp. 2943-2953.

Steinle, S., Reis, S., and Sabel, C. E. (2013). "Quantifying human exposure to air pollution-Moving from static monitoring to spatiotemporally resolved personal exposure assessment". In: Science of the Total Environment 443, pp. 184-193.

Tong, C. and Wong, S. (2000). "A predictive dynamic traffic assignment model in congested capacity-constrained road networks". In: Transportation Research Part B: Methodological 34.8, pp. 625644 .

Trafikverket (2012). Handbok för vägtrafikens luftföroreningar. Kapitel 6, Emissionsdatabaser.

Treiber, M. and Helbing, D. (2002). "Reconstructing the spatio-temporal traffic dynamics from stationary detector data". In: Cooper@ tive Tr@nsport@tionDyn@mics 1.3,pp.3-1.

Treiber, M. and Kesting, A. (2013). "Traffic flow dynamics". In: Traffic Flow Dynamics: Data, Models and Simulation, Springer-Verlag Berlin Heidelberg.

Tsanakas, N., Ekström, J., and Olstam, J. (2017). "Reduction of errors when estimating emissions based on static traffic model outputs". In: Transportation Research Procedia 22, pp. 440-449.

UNECE (2014). Guidelines for reporting emissions and projections data under the Convention on Long-range Transboundary Air Pollution. Tech. rep. ECE/EB.AIR/125. ISSN 1977-8449. United Nations Economic Commission for Europe. 
Vardoulakis, S., Fisher, B. E., Pericleous, K., and Gonzalez-Flesca, N. (2003). "Modelling air quality in street canyons: a review". In: Atmospheric environment 37.2, pp. 155-182.

Vitali, L., Monforti, F., Bellasio, R., Bianconi, R., Sachero, V., Mosca, S., and Zanini, G. (2006). "Validation of a Lagrangian dispersion model implementing different kernel methods for density reconstruction". In: Atmospheric Environment 40.40, pp. 8020-8033.

Wageningen-Kessels, F. v., Lint, H. v., Vuik, K., and Hoogendoorn, S. (2014). "Genealogy of traffic flow models". en. In: EURO Journal on Transportation and Logistics, pp. 1-29.

Wang, M., Daamen, W., Hoogendoorn, S., and Van Arem, B. (2011). "Estimating acceleration, fuel consumption, and emissions from macroscopic traffic flow data". In: Transportation Research Record: Journal of the Transportation Research Board 2260, pp. 123-132.

Wardrop, J. G. (1952). "ROAD PAPER. SOME THEORETICAL ASPECTS OF ROAD TRAFFIC RESEARCH." In: Proceedings of the institution of civil engineers 1.3, pp. 325-362.

Wiedemann, R. (1973). "Simulation des Strassenverkehrsflusses". German. PhD thesis. Karlsruhe: Inst. f. Verkehrswesen d. Univ. Karlsruhe.

Wismans, L., Brink, R. M. M. van de, Brederode, L. J. N., Zantema, K. J., and Berkum, E. C. van (2013). "Comparison of estimation of emissions based on static and dynamic traffic assignment." In: Proceedings of the 93rd Annual Meeting of the Trasportation Research Board, Washington, D.C., USA.

Work, D. B., Blandin, S., Tossavainen, O.-P., Piccoli, B., and Bayen, A. M. (2010). "A traffic model for velocity data assimilation". In: Applied Mathematics Research eXpress 2010.1, pp. 1-35.

Yperman, I. (2007). "The Link Transmission Model for dynamic network loading". PhD thesis. Belgium: Katholieke Universiteit Leuven.

Zeger, S. L., Thomas, D., Dominici, F., Samet, J. M., Schwartz, J., Dockery, D., and Cohen, A. (2000). "Exposure measurement error in time-series studies of air pollution: concepts and consequences." In: Environmental health perspectives 108.5, p. 419. 
Zhong, M., Lingras, P., and Sharma, S. (2004). "Estimation of missing traffic counts using factor, genetic, neural, and regression techniques". In: Transportation Research Part C: Emerging Technologies 12.2, pp. 139-166.

Zhu, Y., Hinds, W. C., Kim, S., and Sioutas, C. (2002). "Concentration and size distribution of ultrafine particles near a major highway". In: Journal of the air \& waste management association 52.9, pp. 1032-1042.

Ziliaskopoulos, A. K. (2000). "A linear programming model for the single destination system optimum dynamic traffic assignment problem". In: Transportation science 34.1, pp. 37-49. 


\section{FACULTY OF SCIENCE AND ENGINEERING}

Linköping Studies in Science and Technology, Licentiate Thesis No. 1835, 2019

Department of Science and Technology

Linköping University

SE-581 83 Linköping, Sweden

www.liu.se 